\title{
SOME ASPECTS OF THE KOBAYASHI AND CARATHÉODORY METRICS ON PSEUDOCONVEX DOMAINS
}

\author{
PRACHI MITTAL AND KAUSHAL VERMA
}

\begin{abstract}
The purpose of this article is to consider two themes both of which emanate from and involve the Kobayashi and the Carathéodory metric. First we study the biholomorphic invariant introduced by B. Fridman on strongly pseudoconvex domains, on weakly pseudoconvex domains of finite type in $\mathbf{C}^{2}$ and on convex finite type domains in $\mathbf{C}^{n}$ using the scaling method. Applications include an alternate proof of the Wong-Rosay theorem, a characterization of analytic polyhedra with noncompact automorphism group when the orbit accumulates at a singular boundary point and a description of the Kobayashi balls on weakly pseudoconvex domains of finite type in $\mathbf{C}^{2}$ and convex finite type domains in $\mathbf{C}^{n}$ in terms of Euclidean parameters. Second a version of Vitushkin's theorem about the uniform extendability of a compact subgroup of automorphisms of a real analytic strongly pseudoconvex domain is proved for $C^{1}$ isometries of the Kobayashi and Carathéodory metrics on a smoothly bounded strongly pseudoconvex domain.
\end{abstract}

\section{INTRODUCTION}

Let $X$ be a Kobayashi hyperbolic complex manifold of dimension $n$ and $\mathcal{H} \subset \mathbf{C}^{n}$ a bounded homogeneous domain. B. Fridman in [14] constructed an interesting non-negative continuous function on $X$ whose value at a given $p \in X$ essentially measures the largest Kobayashi ball around $p$ that is comparable with $\mathcal{H}$. To be more specific take $\mathcal{H}=\mathbf{B}^{n}$ the unit ball in $\mathbf{C}^{n}$ and let $B_{X}(p, r)$ denote the ball in the Kobayashi metric with radius $r>0$ around $p \in X$. Since $X$ is hyperbolic the topology induced by the Kobayashi metric is equivalent to the intrinsic topology on it. Thus for small $r>0, B_{X}(p, r)$ is contained in a coordinate chart around $p$ and hence there is a biholomorphic imbedding $f: \mathbf{B}^{n} \rightarrow X$ such that $f\left(\mathbf{B}^{n}\right) \supset B_{X}(p, r)$. Let $\mathcal{R}$ denote the set of all $r>0$ such that there exists a biholomorphic imbedding $f: \mathbf{B}^{n} \rightarrow X$ with $f\left(\mathbf{B}^{n}\right) \supset B_{X}(p, r) . \mathcal{R}$ is evidently non-empty as explained before. Define

$$
h_{X}\left(p, \mathbf{B}^{n}\right)=\inf _{r \in \mathcal{R}} \frac{1}{r}
$$

which is a non-negative, real valued function on $X$. If $Y$ is another such hyperbolic manifold and $g: X \rightarrow Y$ a biholomorphism then $g$ preserves the balls in the Kobayashi metrics on $X, Y$ and hence $h_{X}\left(p, \mathbf{B}^{n}\right)=h_{Y}\left(g(p), \mathbf{B}^{n}\right)$ which says that $h_{X}\left(p, \mathbf{B}^{n}\right)$ is a biholomorphic invariant. The function $h_{X}(p, \mathcal{H})$ will henceforth be referred to as Fridman's invariant and while the same construction holds for any invariant metric (provided of course when the intrinsic topology on $X$ coincides with that induced by the invariant metric), the Kobayashi and the Carathéodory metric shall be exclusively dealt with here. In fact, Fridman's invariant defined using the Carathéodory metric appears only in two places one at the end of the section 4 which considers strongly pseudoconvex domains where this metric is most tractable and second in section 6 where convex domains are considered on which the Carathéodory metric equals the Kobayashi metric by Lempert's work. Barring these exceptions, the Kobayashi metric is the one that is considered. The choice of metric will be clarified often enough to avoid ambiguities for the same notation will be used for this invariant; the choice of $\mathcal{H}$ will be made explicit though. Having defined this let us recall some of its basic properties proved in 14. Among other things, it was shown that when $X$ is Kobayashi hyperbolic then $p \mapsto h_{X}(p, \mathcal{H})$ is continuous and if there exists $p^{0} \in X$ such that $h_{X}\left(p^{0}, \mathcal{H}\right)=0$ then $h_{X}(p, \mathcal{H}) \equiv 0$ for all $p \in X$ and moreover $X$ is biholomorphic to $\mathcal{H}$. This is reminiscent of the $C / K$ invariant, i.e., the ratio of the Carathéodory and the Eisenman-Kobayashi volume form, used in 39. Indeed, this ratio is at most one for a bounded domain and equals one at some point if and only if the domain is equivalent to $\mathbf{B}^{n}$. It was also shown that if $D \subset C^{n}$ is a $C^{3}$-smooth strongly

1991 Mathematics Subject Classification. Primary: 32F45 ; Secondary : 32Q45.

The second author was supported in part by a grant from UGC under DSA-SAP, Phase IV. 
pseudoconvex domain equipped with the Kobayashi metric such that $D$ is not biholomorphic to $\mathbf{B}^{n}$, then $h_{D}\left(p, \mathbf{B}^{n}\right) \rightarrow 0$ as $p \rightarrow \partial D$. The proof of this crucially used the fact that the model domain at strongly pseudoconvex boundary points is the ball. As a consequence if $p^{0} \in \partial D$ and $U$ is an open neighbourhood of $p^{0}$ then using the automorphism group of $\mathbf{B}^{n}$ it is possible to find a sequence of holomorphic mappings $F^{j}: U \cap D \rightarrow \mathbf{B}^{n}$ that are biholomorphic onto their images which exhaust $\mathbf{B}^{n}$, i.e., for every compact $K \subset \mathbf{B}^{n}$ there is some $m=m(K)$ such that $K \subset F_{m}(U \cap D)$.

The first goal of this work is to understand Fridman's invariant on a broader class of domains, more specifically the weakly pseudoconvex finite type domains in $\mathbf{C}^{2}$ and convex domains of finite type in $\mathbf{C}^{n}$. The problem is of course to determine the behaviour of $h_{D}(p, \mathcal{H})$ as $p \rightarrow \partial D$ and it is natural to try and apply the scaling method to understand this question on more general domains. Any attempt to do this must take into account the fact that model domains at boundary points are not unique unlike the strongly pseudoconvex case and hence the boundary limits of $h_{D}(p, \mathcal{H})$ will depend on the nature of approach to the boundary point. Indeed, in general the model domains for a smooth weakly pseudoconvex finite type domain in $\mathbf{C}^{2}$ and convex finite type domains in $\mathbf{C}^{n}$ are given by

$$
\rho(z)=2 \Re z_{2}+P_{2 m}\left(z_{1}, \bar{z}_{1}\right)
$$

where $P_{2 m}\left(z_{1}, \bar{z}_{1}\right)$ is a homogeneous subharmonic polynomial of degree $2 m$, and

$$
\rho(z)=2 \Re z_{n}+Q_{2 m}\left({ }^{\prime} z,^{\prime} \bar{z}\right)
$$

where ${ }^{\prime} z=\left(z_{1}, z_{2}, \ldots, z_{n-1}\right)$ and $Q_{2 m}\left({ }^{\prime} z,^{\prime} \bar{z}\right)$ is a convex polynomial of degree at most $2 m$ respectively. More can be said about the polynomials $P_{2 m}\left(z_{1}, \bar{z}_{1}\right)$ and $Q_{2 m}\left({ }^{\prime} z,^{\prime} \bar{z}\right)$ in case the approach is non-tangential. Since the definition of $h_{D}(p, \mathcal{H})$ involves the Kobayashi/Carathéodory balls (which are global objects vis-a-vis the infinitesimal metric), this approach quickly leads to considerations that involve the Kobayashi/Carathéodory balls on the scaled domains and their convergence to the corresponding balls in the limit domain. Viewed differently, this is a stability problem for the integrated distance and not its infinitesimal version about which much is known - see [18] and [41] for instance.

Theorem 1.1. Let $D \subset \mathbf{C}^{n}$ be a bounded domain, $p^{0} \in \partial D$ and let $\left\{p^{j}\right\} \subset D$ be a sequence that converges to $p^{0}$. Then the boundary behaviour of $h_{D}\left(p, \mathbf{B}^{n}\right)$ along $\left\{p^{j}\right\}$ can be described in the following cases:

(i) If $D$ is $C^{2}$-smooth strongly pseudoconvex equipped with either the Kobayashi or the Carathéodory metric, then $h_{D}\left(p^{j}, \mathbf{B}^{n}\right) \rightarrow 0$.

(ii) If $D$ is $C^{\infty}$-smooth weakly pseudoconvex of finite type in $\mathbf{C}^{2}$ equipped with the Kobayashi metric, then $h_{D}\left(p^{j}, \mathbf{B}^{2}\right) \rightarrow h_{D_{\infty}}\left((0,-1), \mathbf{B}^{2}\right)$ where $D_{\infty}$ is the model domain defined by

$$
D_{\infty}=\left\{z=\left(z_{1}, z_{2}\right) \in \mathbf{C}^{2}: 2 \Re z_{2}+P_{2 m}\left(z_{1}, \bar{z}_{1}\right)<0\right\},
$$

and $P_{2 m}\left(z_{1}, \bar{z}_{1}\right)$ is a subharmonic polynomial of degree at most $2 m(m \geq 1)$ without harmonic terms, $2 m$ being the 1-type of $\partial D$ near $p^{0}$.

(iii) If $D$ is $C^{\infty}$-smooth convex of finite type equipped with the Kobayashi metric (which equals the Carathéodory metric), then $h_{D}\left(p^{j}, \mathbf{B}^{n}\right) \rightarrow h_{D_{\infty}}\left(\left(^{\prime} 0,-1\right), \mathbf{B}^{n}\right)$ where $D_{\infty}$ is the model domain defined by

$$
D_{\infty}=\left\{z=\left({ }^{\prime} z, z_{n}\right) \in \mathbf{C}^{n}: 2 \Re z_{n}+Q_{2 m}\left({ }^{\prime} z{ }^{\prime} \bar{z}\right)<0\right\},
$$

and $Q_{2 m}\left({ }^{\prime} z,^{\prime} \bar{z}\right)$ is a non-degenerate convex polynomial of degree at most $2 m(m \geq 1), 2 m$ being the 1-type of $\partial D$ near $p^{0}$.

The proof of this theorem is contained in sections 4,5 and 6 . While this is a global result, it turns out that $h_{D}\left(\cdot, \mathbf{B}^{n}\right)$ can be localised much like the Kobayashi or the Carathéodory metric near peak points and this is done in proposition 3.4 . Therefore there is a completely natural formulation of this result that is of a local nature and this will be evident from the proof of this theorem.

Studying this invariant using the scaling procedure unifies several disparate questions and applications include an alternate proof of the Wong-Rosay theorem - it must be mentioned that this was also done by B. Fridman in [14] using the fact that $h_{D}\left(p, \mathbf{B}^{n}\right) \rightarrow 0$ as $p \rightarrow \partial D$ for $D$ a strongly pseudoconvex domain, the emphasis here being a different approach, a characterization of normal analytic polyhedra with a noncompact automorphism group with one orbit accumulating at a singular boundary point (and this recovers part of the main theorem in [24]), and theorems of Coupet-Pinchuk-Sukhov ([10]) and Pinchuk ([30]) about the inequivalence of two given domains with different Levi geometry. 
As another byproduct, it is also possible to describe the Kobayashi balls on a weakly pseudoconvex finite type domains in $\mathbf{C}^{2}$ and on convex finite type domains in $\mathbf{C}^{n}$ in Euclidean terms. More precisely, it is known that on a strongly pseudoconvex domain $D$ in $\mathbf{C}^{n}, B_{D}(p, R)$ contains and is contained in ellipsoids $E_{p}^{ \pm}$each of whose major and minor axis are of the order of $C(R)(\operatorname{dist}(p, \partial D))^{1 / 2}$ and $c(R) \operatorname{dist}(p, \partial D)$ respectively where the positive constants $C(R)$ and $c(R)$ are independent of $p$. It is possible to obtain analogues of this result for weakly pseudoconvex and convex finite type domains without integrating the infinitesimal metric. Such estimates were obtained by Aladro in 1 for weakly pseudoconvex finite type domains in $\mathbf{C}^{2}$ using a suitable metric on the horizontal subbundle on $\partial D$ due to Nagel-Stein-Wainger [29]. The arguments used here avoid any reference to such considerations and instead rely only on scaling. These estimates, as is known (see for example [25]), are useful in verifying the generalized sub-mean value property for plurisubharmonic functions - a property that is needed in proving analogues of Fatou's theorem on the boundary behaviour of $H^{p}$ functions.

The second theme explored in this article is the rigidity of continuous isometries of these metrics. More precisely if $D, D^{\prime}$ are $C^{2}$-smooth strongly pseudoconvex domains in $\mathbf{C}^{n}$ and $f: D \rightarrow D^{\prime}$ is a continuous isometry of the Kobayashi metrics on $D, D^{\prime}$, it is not known whether $f$ must necessarily be holomorphic or conjugate holomorphic. The same question can be asked for the Carathéodory metric or for that matter any invariant metric as well. An affirmative answer for the Bergman metric was given in [17] and this required knowledge of the limiting behaviour of the holomorphic sectional curvatures of the Bergman metric near strongly pseudoconvex points. In general, the Kobayashi metric is just upper semicontinuous and therefore a different approach will be needed for this question. The case of continuous isometries when $D$ is smooth strongly convex and $D^{\prime}$ is the unit ball was dealt with in [35] and this was improved upon in 22 to handle the case when $D$ is a $C^{2, \epsilon}$-smooth strongly pseudoconvex domain $(\epsilon>0)$, and a common ingredient in both proofs was the use of Lempert discs. Motivated by such considerations it seemed natural to determine the extent to which isometries behave like holomorphic mappings and one example is provided by the following:

Theorem 1.2. Let $D_{1}, D_{2}$ be two bounded strongly pseudoconvex domains in $\mathbf{C}^{n}$ with $C^{2}$-smooth boundaries. Let $D_{1}^{k}, D_{2}^{k}$ for $k \geq 1$ be two sequences of domains that converge to $D_{1}, D_{2}$ respectively in the $C^{2}$ topology and let $d_{D_{1}^{k}}, d_{D_{2}^{k}}$ denote the Kobayashi metrics on these domains. Similarly let $d_{D_{1}}, d_{D_{2}}$ denote the Kobayashi metrics on $D_{1}, D_{2}$ respectively. Suppose that $f^{k}:\left(D_{1}^{k}, d_{D_{1}^{k}}\right) \rightarrow\left(D_{2}^{k}, d_{D_{2}^{k}}\right)$ is a $C^{1}$-smooth isometry for each $k \geq 1$ and that there is a point $p^{1} \in D_{1}$ such that some subsequence $\left\{f^{k_{j}}\left(p^{1}\right)\right\}$ converges to a point $p^{2} \in D_{2}$. Then there is a uniform constant $C>0$ with the property that:

$$
\left|f^{k_{j}}(p)-f^{k_{j}}(q)\right| \leq C|p-q|^{1 / 2}
$$

for all $p, q \in D_{1}$.

Several remarks are in order here. First, by a $C^{0}$-isometry we mean a distance preserving bijection between the metric spaces $\left(D_{1}, d_{D_{1}}\right)$ and $\left(D_{2}, d_{D_{2}}\right)$. For $k \geq 1$, a $C^{k}$-isometry is a $C^{k}$-diffeomorphism $f$ from $D_{1}$ onto $D_{2}$ with $f^{*}\left(F_{D_{1}}\right)=F_{D_{2}}$. Second, a given pair of points $p, q \in D_{1}$ are evidently contained in $D_{1}^{k}$ for all large $k$ and hence $f^{k_{j}}(p), f^{k_{j}}(q)$ are well defined. Thirdly, the estimate shows that the family $\left\{f^{k_{j}}\right\}$ is 'uniformly equicontinuous' on $\bar{D}_{1}$, and this may be regarded as a version of Vitushkin's theorem about the uniform extendability of a compact subgroup of holomorphic automorphisms of a smooth real analytic strongly pseudoconvex domain for isometries. The hypothesis about the existence of $p^{1} \in D_{1}$ such that $f^{k_{j}}\left(p^{1}\right) \rightarrow p^{2} \in D_{2}$ is to be understood as saying that $\left\{f^{k_{j}}\right\}$ is a compact family. Without this the theorem is false even in the holomorphic category as the example of the unit ball shows. It must be mentioned that other relevant theorems of this nature for holomorphic automorphisms were proved by Coupet ([6]) and Coupet-Sukhov ([1]). When no such $p^{1}, p^{2}$ exist for any subsequence of $f^{k}$, that corresponds to the non-compact situation and this has been studied by Kim-Krantz in 22] where they prove that $D_{1}, D_{2}$ are both biholomorphic to the ball provided their boundaries are at least $C^{2, \epsilon}$ smooth where $\epsilon>0$. This statement can be deduced from their arguments in 22] without any additional difficulties. The main step in the proof of the above theorem is to show that

$$
\left|d f^{k}(z) v\right| \lesssim \frac{|v|}{\left(\operatorname{dist}\left(z, \partial D_{1}^{k}\right)\right)^{1 / 2}}
$$


uniformly for all $k$, vectors $v \in \mathbf{C}^{n}$ and $z \in D_{1}^{k}$. This in turn relies on knowing that $f^{k}$ uniformly preserves the distance to the boundary, i.e.,

$$
\operatorname{dist}\left(f^{k}(z), \partial D_{2}^{k}\right) \approx \operatorname{dist}\left(z, \partial D_{1}^{k}\right)
$$

for $z \in D_{1}^{k}$. In the holomorphic case, this would be done by pulling back a strongly plurisubharmonic defining function for $D_{2}^{k}$ by $f^{k}$ and applying the Hopf lemma to this composition. This cannot be apriori applied in the case of isometries and to circumvent the difficulty, the variation of the integrated Kobayashi distance in the domains $D_{1}^{k}, D_{2}^{k}$ must be studied. This is done in lemma 9.7 and lemma 9.8 and these yield the $C^{2}$-stability of the estimates for the integrated Kobayashi distance (cf proposition 9.1 and 9.2) obtained by Forstneric and Rosay in [13. The proof concludes by integrating (1.2) exactly as in the case of holomorphic mappings.

Some of the material presented here has benefitted from conversations that the first author had with KangTae Kim and Rasul Shafikov. We would like to thank them for their valuable comments, suggestions and encouragement.

\section{Notation And Terminology}

Let $\Delta$ denote the open unit disc in the complex plane and let $d_{h y p}(a, b)$ denote the distance between two points $a, b \in \Delta$ with respect to the hyperbolic metric. For $r>0, \Delta(0, r) \subset \mathbf{C}$ will be the disc of radius $r$ around the origin and $B(z, \delta) \subset \mathbf{C}^{n}$ will be the Euclidean ball of radius $\delta>0$ around $z$. Let $X$ be a complex manifold of dimension $n$. The Kobayashi and the Carathéodory distances on $X$, denoted by $d_{X}$ and $c_{X}$ respectively, are defined as follows:

Let $z \in X$ and fix $\xi$ a holomorphic tangent vector at $z$. Define the associated infinitesimal Carathéodory and Kobayashi metrics as

$$
\begin{aligned}
F_{X}^{C}(z, \xi) & =\sup \{|d f(z) \xi|: f \in \mathcal{O}(X, \Delta)\} \\
\text { and } \quad F_{X}^{K}(z, \xi) & =\inf \left\{\frac{1}{\alpha}: \alpha>0, f \in \mathcal{O}(\Delta, X) \text { with } f(0)=z, f^{\prime}(0)=\alpha \xi\right\}
\end{aligned}
$$

respectively. The Kobayashi length of a piecewise $\mathcal{C}^{1}$-curve $\gamma:[0,1] \rightarrow X$ is given by

$$
L_{X}(\gamma)=\int_{0}^{1} F_{X}^{K}(\gamma(t), \dot{\gamma}(t)) d t
$$

and finally the Kobayashi distance between $p, q \in X$ is defined as

$$
d_{X}(p, q)=\inf L_{X}(\gamma)
$$

where the infimum is taken over all piecewise differentiable curves $\gamma$ in $X$ joining $p$ to $q$. Recall that $X$ is taut if $\mathcal{O}(\Delta, X)$ is a normal family.

The Carathéodory distance $c_{X}$ between $p, q \in X$ is defined by setting

$$
c_{X}(p, q)=\sup _{f} d_{h y p}((f(p), f(q))
$$

where the supremum is taken over the family of all holomorphic mappings $f: X \rightarrow \Delta$.

The notion of finite type for a real-analytic hypersurface $M \subset \mathbf{C}^{n}$ will be in the sense of D'Angelo, i.e., there is no germ of a positive dimensional subvariety in $M$.

\section{SOME REMARKS ON FRIDMAN'S INVARIANT}

In this section we gather and prove several basic properties of $h_{X}(p, \mathcal{H})$ that will be used in the sequel. This will be done in a slightly more general setting by replacing the homogeneous domain $\mathcal{H}$ by a Kobayashi hyperbolic domain $\Omega \subset \mathbf{C}^{n}$ such that the quotient $\Omega / \operatorname{Aut}(\Omega)$ is compact, where as usual $\operatorname{Aut}(\Omega)$ denotes the group of holomorphic automorphisms of $\Omega$ and as before $X$ will be Kobayashi hyperbolic. An analogue of (1.1) can thus be defined as

$$
h_{X}(p, \Omega)=\inf _{r \in \mathcal{R}} \frac{1}{r}
$$


where $\mathcal{R}$ denotes the set of all $r>0$ with the property that there is a biholomorphic imbedding $f: \Omega \rightarrow X$ with $f(\Omega) \supset B_{X}(p, r)$.

We begin with the following version of lemma 1.1 of 14 which will be crucial for our purposes:

Lemma 3.1. Let $X$ be a Kobayashi hyperbolic manifold of complex dimension $n$ and let $D$ be a taut domain in $\mathbf{C}^{n}$. Suppose that there exist two relatively compact sets $K_{1} \subset D$ and $K_{2} \subset X$ and a sequence $\left\{F^{k}\right\}$ of mappings $F^{k}: D \rightarrow F^{k}(D) \subset X$ satisfying the following conditions:

(i) for each $k \geq 1, F^{k}: D \rightarrow F^{k}(D)$ is a biholomorphism,

(ii) for each $k \geq 1$, there exists a point $z^{k} \in K_{1}$ such that $F^{k}\left(z^{k}\right) \in K_{2}$,

(iii) for any compact $L \subset X$ there exists a number $s=s(L)$ such that $L \subset F^{s}(D)$.

Then $X$ is biholomorphically equivalent to $D$.

Proof. Consider $\phi^{k}=\left(F^{k}\right)^{-1}: F^{k}(D) \rightarrow D$ and let $\left\{U_{n}\right\}$ be an exhaustion of $X$ by relatively compact submanifolds such that $U_{n}$ is compactly contained in $U_{n+1}$ for all $n$. In view of the tautness of $D$ and (ii), we may assume that some subsequence of $\left\{\phi^{k}\right\}$ (which we continue to denote by the same symbols) satisfies the following condition: for each $n \geq 1$, there exists a $N$ such that $\left\{\phi^{k}\right\}_{k \geq N}$ is defined on $U_{n}$ and converges uniformly on compact sets of $U_{n}$ to $\phi: U_{n} \rightarrow D$. Indeed, $\phi: X \rightarrow D$. We show that $\phi$ is a biholomorphism from $X$ onto $D$.

To prove the injectivity of $\phi$ consider $x^{1}$ and $x^{2}$ any two points in $X$. Then for each $k$,

$$
d_{X}\left(x^{1}, x^{2}\right)=d_{X}\left(F^{k} \circ \phi^{k}\left(x^{1}\right), F^{k} \circ \phi^{k}\left(x^{2}\right)\right) .
$$

It follows from the distance non-increasing property of the holomorphic mappings that

$$
d_{X}\left(F^{k} \circ \phi^{k}\left(x^{1}\right), F^{k} \circ \phi^{k}\left(x^{2}\right)\right) \leq d_{D}\left(\phi^{k}\left(x^{1}\right), \phi^{k}\left(x^{2}\right)\right)
$$

and by using the triangle inequality, we get

$$
d_{D}\left(\phi^{k}\left(x^{1}\right), \phi^{k}\left(x^{2}\right)\right) \leq d_{D}\left(\phi^{k}\left(x^{1}\right), \phi\left(x^{1}\right)\right)+d_{D}\left(\phi\left(x^{1}\right), \phi\left(x^{2}\right)\right)+d_{D}\left(\phi\left(x^{2}\right), \phi^{k}\left(x^{2}\right)\right) .
$$

Combining (3.2), (3.3) and (3.4) and letting $k \rightarrow \infty$ gives

$$
d_{X}\left(x^{1}, x^{2}\right) \leq d_{D}\left(\phi\left(x^{1}\right), \phi\left(x^{2}\right)\right) .
$$

Since $X$ is hyperbolic, we must have $x^{1}=x^{2}$ whenever $\phi\left(x^{1}\right)=\phi\left(x^{2}\right)$.

It remains to show that $\phi$ is surjective. For this note that we have already proved that $X$ is biholomorphically equivalent to a domain $\phi(X) \subset D$. Hence we can consider as $X$ as a subdomain of a taut domain $D$. This together with (ii) implies that $\left\{F^{k}\right\}$ admits a subsequence that converges uniformly on compacts of $D$ and since $F^{k}(D)$ exhaust $X$, the limit mapping $F: D \rightarrow \bar{X}$. In view of relative compactness of $K_{1}$ and $K_{2}$, we may assume that (after passing to a subsequence if necessary) that $\left\{F^{k}\left(z^{k}\right)\right\}$ converges to $x^{0} \in \overline{K_{2}}$ and $\left\{z^{k}\right\}$ to $z^{0} \in \overline{K_{1}}$. It is immediate that $F\left(z^{0}\right)=x^{0}$. Now using the hyperbolicity of $X$, choose $\epsilon>0$ small enough so that $B_{X}\left(x^{0}, \epsilon\right)$ is compactly contained in $X$. Then it follows from (iii) that $B_{X}\left(x^{0}, \epsilon\right) \subset F^{k}(D)$ for all $k$ large. This implies that $B_{X}\left(x^{0}, \epsilon\right) \subset F(D)$.

Let $\mathrm{Jac} F^{k}$ be the Jacobian of $F^{k}$. Applying Hurwitz's theorem to the sequence $\left\{\mathrm{Jac} F^{k}\right\}$, we deduce that either the Jacobian of $F$ is never zero at any point of $D$ or it is identically zero on $D$. In the latter case, $F(D)$ cannot contain any open set. Since $F(D) \supset B_{X}\left(x^{0}, \epsilon\right)$, we conclude that $F(D)$ is open. In particular, $F(D) \subset X$. For the mapping $\phi \circ F: D \rightarrow D$ and any $z \in D$, it follows that

$$
\phi \circ F(z)=\lim _{k \rightarrow \infty} \phi^{k} \circ F^{k}(z)=z
$$

This shows that $D \subset \phi(X)$. This completes the proof of the lemma.

Lemma 3.2. Let $\Omega$ be a hyperbolic domain in $\mathbf{C}^{n}$ and assume that $\Omega / A u t(\Omega)$ is compact. Then $\Omega$ is complete hyperbolic and hence taut.

Proof. By hypothesis there exists a compact set $K \subset \Omega$ such that for any point $x \in \Omega$ there exists a $y \in K$ and a $g \in \operatorname{Aut}(\Omega)$ with $g(y)=x$. Let $\epsilon>0$ be sufficiently small so that

$$
L=\left\{z \in \Omega: d_{\Omega}(z, K)<\epsilon\right\}
$$


is compactly contained in $\Omega$. Let $\left\{p^{k}\right\}$ be a Cauchy sequence in $\Omega$ in the Kobayashi metric. Then there exists a positive integer $k_{0}$ such that $d_{\Omega}\left(p^{k}, p^{k_{0}}\right)<\epsilon$ for $k \geq k_{0}$. Let $g \in \operatorname{Aut}(\Omega)$ be such that $g\left(p^{k_{0}}\right) \in K$. Since biholomorphisms are isometries for the Kobayashi metric, it follows that

$$
d_{\Omega}\left(g\left(p^{k}\right), g\left(p^{k_{0}}\right)\right)=d_{\Omega}\left(p^{k}, p^{k_{0}}\right)<\epsilon
$$

which shows that $g\left(p^{k}\right) \in L$ for all $k \geq k_{0}$ and that $\left\{g\left(p^{k}\right)\right\}$ is also Cauchy in the Kobayashi metric. Since $L$ is compactly contained in $\Omega$ and $\Omega$ is hyperbolic, there exists a $q \in L$ such that $g\left(p^{k}\right) \rightarrow q$ as $k \rightarrow \infty$. It follows that $p^{k} \rightarrow g^{-1}(q)$ as $k \rightarrow \infty$. This shows that $\Omega$ is complete.

Proposition 3.3. Let $X$ be a Kobayashi hyperbolic manifold of complex dimension $n$ and let $\Omega$ be a hyperbolic domain in $\mathbf{C}^{n}$ such that $\Omega / A u t(\Omega)$ is compact. Then

(i) if there is an $x^{0} \in X$ such that $h_{X}\left(x^{0}, \Omega\right)=0$, then $h_{X}\left(x^{0}, \Omega\right) \equiv 0$ and $X$ is biholomorphically equivalent to $\Omega$.

(ii) if there is an $x^{0} \in X$ such that $h_{X}\left(x^{0}, \Omega\right)>0$ and $X$ is taut, then there exists a biholomorphic imbedding $F: \Omega \rightarrow X, F(\Omega) \supseteq B_{X}\left(x^{0}, r\right)$ such that $h_{X}\left(x^{0}, \Omega\right)=1 / r$ (i.e., the variational problem has an extremal).

(iii) $h_{X}(\cdot, \Omega)$ is continuous on $X$.

Proof. To prove (i) let $x^{0} \in X$ be such that $h_{X}\left(x^{0}, \Omega\right)=0$. By definition, there exists a sequence $\left\{\phi^{k}\right\}$ of biholomorphic imbeddings, $\phi^{k}: \Omega \rightarrow X$ such that $B_{X}\left(x^{0}, k\right) \subset \phi^{k}(\Omega)$. Let $K$ be a compact subset of $\Omega$ such that for every $y$ in $\Omega$ there exists a $x \in K$ and a $g \in \operatorname{Aut}(\Omega)$ satisfying $g(y)=x$. Composing with an appropriate automorphism of $\Omega$, if necessary, we may assume that $\left(\phi^{k}\right)^{-1}\left(x^{0}\right)=q^{k} \in K$. We now claim that $\left\{\phi^{k}(\Omega)\right\}$ is a sequence of subdomains of $X$ that exhausts $X$. Indeed, for any compact $L \subset X$; since $d_{X}(\cdot, \cdot)$ is continuous, there exists a constant $c=c(L)>0$ such that $d_{X}\left(x^{0}, x\right) \leq c$ for all $x \in L$. It follows that $L \subset B_{X}\left(x^{0}, k\right) \subset \phi^{k}(\Omega)$ for all $k \geq c$. Now applying lemma 3.1 to the sequence $\left\{\phi^{k}\right\}$ with $K_{1}=\left\{q^{k}\right\}$ and $K_{2}=\left\{x^{0}\right\}$, we obtain that $X$ is biholomorphically equivalent to $\Omega$. Let $f: X \rightarrow \Omega$ be a biholomorphism from $X$ onto $\Omega$. Then for any $x \in X, h_{X}(x, \Omega)=h_{\Omega}(f(x), \Omega)=0$. Hence, $h_{X}(\cdot, \Omega) \equiv 0$.

For (ii) observe that by definition, there exists a sequence of biholomorphic imbeddings, $F^{k}: \Omega \rightarrow X$ and $R_{k}>0$ satisfying $B_{X}\left(x^{0}, R_{k}\right) \subset F^{k}(\Omega)$ and $\left(R_{k}\right)^{-1} \rightarrow h_{X}\left(x^{0}, \Omega\right)$ as $k \rightarrow \infty$. Let $K \subset \Omega$ be as in part (i). Then for each $k$, there exists a $f^{k} \in \operatorname{Aut}(\Omega)$ such that $f^{k}\left(\left(F^{k}\right)^{-1}\left(x^{0}\right)\right)=q^{k} \in K$. Set $\tilde{F}^{k}:=F^{k} \circ\left(f^{k}\right)^{-1}: \Omega \rightarrow X$. Since $X$ is taut, it follows that $\left\{\tilde{F}^{k}\right\}$ is a normal family. Hence, $\tilde{F}^{k}$ admits a subsequence that converges uniformly on compact sets of $\Omega$ to a holomorphic mapping $F: \Omega \rightarrow X$ or $F \equiv \infty$. The latter cannot be true since $\tilde{F}^{k}\left(q^{k}\right)=x^{0}$ which implies that $F(q)=x^{0}$ where $q=\lim _{k \rightarrow \infty} q^{k} \in K$. We must therefore have a holomorphic mapping $F: \Omega \rightarrow X$.

To establish the injectivity of $F$ fix $\epsilon>0$ arbitrarily small. Then $B_{X}\left(x^{0},\left(h_{X}\left(x^{0}, \Omega\right)\right)^{-1}-\epsilon\right) \subset$ $B_{X}\left(x^{0}, R_{k}\right) \subset \tilde{F}^{k}(\Omega)$ for all $k$ large. It follows that $B_{X}\left(x^{0},\left(h_{X}\left(x^{0}, \Omega\right)\right)^{-1}-\epsilon\right) \subset F(\Omega)$. Since $F(\Omega)$ contains a non-empty open set, $F$ must be non-constant. Consider any point $c \in \Omega$. Each mapping $\tilde{F}^{k}(\cdot)-\tilde{F}^{k}(c)$ never vanishes in $\Omega \backslash\{c\}$ because of the injectivity of $\tilde{F}^{k}$ in $\Omega$. Applying Hurwitz's theorem to the sequence $\left\{\tilde{F}^{k}(\cdot)-\tilde{F}^{k}(c)\right\} \subset \mathcal{O}\left(\Omega \backslash\{c\}, \mathbf{C}^{n}\right)$, we get that $F(z) \neq F(c)$ for all $z \in \Omega \backslash\{c\}$. Since $c$ is any arbitrary point of $\Omega$, this is just the assertion that $F$ is injective on $\Omega$.

It remains to show that $F(\Omega) \supset B_{X}\left(x^{0},\left(h_{X}\left(x^{0}, \Omega\right)\right)^{-1}\right)$. For this, consider

$$
G^{k}:=\left(\tilde{F}^{k}\right)^{-1}: B_{X}\left(x^{0},\left(h_{X}\left(x^{0}, \Omega\right)\right)^{-1}-\epsilon\right) \rightarrow \Omega
$$

that are defined for all large $k$. Since $\Omega$ is taut, $\left\{G^{k}\right\}$ forms a normal family. Also, $G^{k}\left(x^{0}\right)=$ $q^{k}$ implies that $\left\{G^{k}\right\}$ admits a convergent subsequence that converges uniformly on compact sets of $B_{X}\left(x^{0},\left(h_{X}\left(x^{0}, \Omega\right)\right)^{-1}-\epsilon\right)$ to a holomorphic mapping $G: B_{X}\left(x^{0},\left(h_{X}\left(x^{0}, \Omega\right)\right)^{-1}-\epsilon\right) \rightarrow \Omega$ and $G\left(x^{0}\right)=q$. Now for any $p \in B_{X}\left(x^{0},\left(h_{X}\left(x^{0}, \Omega\right)\right)^{-1}\right)$, there exists a compact set $L \subset \Omega$ such that $G^{k}(p) \subset L$ for all $k$ large. Consequently, $p=\tilde{F}^{k} \circ G^{k}(p) \subset \tilde{F}^{k}(L)$ for all $k$ large. Let $\left\{w^{k}\right\} \subset L$ such that $\tilde{F}^{k}\left(w^{k}\right)=p$. In view of compactness of $L$, there exists a $w^{0} \in L$ such that $w^{k} \rightarrow w^{0}$ as $k \rightarrow \infty$. Since $\tilde{F}^{k} \rightarrow F$ uniformly on compacts of $\Omega$, we have $\tilde{F}^{k}\left(w^{k}\right) \rightarrow F\left(w^{0}\right)$. Hence, $p=F\left(w^{0}\right) \subset F(\Omega)$. Therefore, $F(\Omega) \supset B_{X}\left(x^{0},\left(h_{X}\left(x^{0}, \Omega\right)\right)^{-1}\right)$. 
To prove (iii) let $x^{0} \in X$ be such that $h_{X}\left(x^{0}, \Omega\right)>0$ and consider $H_{X}\left(x^{0}, \Omega\right):=\left(h_{X}\left(x^{0}, \Omega\right)\right)^{-1}$. It is enough to show that $H_{X}(\cdot, \Omega)$ is continuous. Let $x^{1}, x^{2} \in X$ such that $2 d_{X}\left(x^{1}, x^{2}\right)<H_{X}\left(x^{1}, \Omega\right)$. Let $\epsilon>0$ be such that

$$
H_{X}\left(x^{1}, \Omega\right)-2 d_{X}\left(x^{1}, x^{2}\right)-2 \epsilon>0 .
$$

Using the triangle inequality for $d_{X}$, we get

$$
B_{X}\left(x^{2}, H_{X}\left(x^{1}, \Omega\right)-\epsilon-d_{X}\left(x^{1}, x^{2}\right)\right) \subset B_{X}\left(x^{1}, H_{X}\left(x^{1}, \Omega\right)-\epsilon\right)
$$

which means that

$$
H_{X}\left(x^{1}, \Omega\right)-\epsilon-d_{X}\left(x^{1}, x^{2}\right) \leq H_{X}\left(x^{2}, \Omega\right)
$$

or equivalently that

$$
H_{X}\left(x^{1}, \Omega\right)-H_{X}\left(x^{2}, \Omega\right) \leq d_{X}\left(x^{1}, x^{2}\right)+\epsilon .
$$

But by the choice of $\epsilon>0$ we know that

$$
H_{X}\left(x^{2}, \Omega\right)-\epsilon-d_{X}\left(x^{1}, x^{2}\right)>0
$$

and similarly the triangle inequality applied once again gives

$$
B_{X}\left(x^{1}, H_{X}\left(x^{2}, \Omega\right)-\epsilon-d_{X}\left(x^{1}, x^{2}\right)\right) \subset B_{X}\left(x^{2}, H_{X}\left(x^{2}, \Omega\right)-\epsilon\right)
$$

or equivalently that

$$
H_{X}\left(x^{2}, \Omega\right)-H_{X}\left(x^{1}, \Omega\right) \leq d_{X}\left(x^{1}, x^{2}\right)+\epsilon .
$$

Combining (3.5) and (3.6) yields

$$
\left|H_{X}\left(x^{2}, \Omega\right)-H_{X}\left(x^{1}, \Omega\right)\right| \leq d_{X}\left(x^{1}, x^{2}\right)
$$

and this shows that $H_{X}(\cdot, \Omega)$ is continuous in the topology induced by the Kobayashi metric. Since $X$ is hyperbolic, the topology induced by the Kobayashi metric coincides with its intrinsic topology and hence the result follows.

It must be mentioned that corresponding statements for $h_{X}(p, \mathcal{H})$ where $\mathcal{H}$ is a bounded homogeneous domain were proved in [14. A similar situation that dealt with a hyperbolic domain $\Omega$ with $\Omega /$ Aut $(\Omega)$ compact was also considered in [12].

We shall henceforth be concerned primarily with Fridman's invariant as defined in (1.1) using the Kobayashi metric. To analyse its boundary behaviour the first step is to show that it can be localised near peak points.

Proposition 3.4. Let $D \subset \mathbf{C}^{n}$ be a bounded domain and let $z^{0} \in \partial D$ be a local holomorphic peak point. Then for every neighbourhood $U$ of $z^{0}$ there exists a neighbourhood $V \subset U$ of $z^{0}$ with $V$ relatively compact in $U$ such that for all $z \in V \cap D$, we have

$$
c h_{D}\left(z, \mathbf{B}^{n}\right) \leq h_{U \cap D}\left(z, \mathbf{B}^{n}\right) \leq h_{D}\left(z, \mathbf{B}^{n}\right)
$$

where $c>0$ is a constant independent of $z \in V \cap D$.

Proof. Let $U$ be a neighbourhood of $z^{0}$ and $g \in \mathcal{A}(U \cap D)$, the algebra of continuous functions on the closure of $U \cap D$ that are holomorphic on $U \cap D$, such that $g\left(z^{0}\right)=1$ and $|g(p)|<1$ for $p \in \overline{U \cap D} \backslash\left\{z^{0}\right\}$. Fix $\epsilon>0$. Then there exists a neighbourhood $U_{1} \subset U$ of $z^{0}$ such that

$$
F_{D}^{K}(z, v) \leq F_{U \cap D}^{K}(z, v) \leq(1+\epsilon) F_{D}^{K}(z, v)
$$

for $z \in U_{1} \cap D$ and $v$ a tangent vector at $z$. This is possible by the localisation property of the Kobayashi metric (see for example lemma 2 in [33] or [16]).

The first inequality evidently implies that $B_{U \cap D}(z, r) \subset B_{D}(z, r)$ for all $z \in U_{1} \cap D$ and all $r>0$. Let $R>0$ be such that there exists a biholomorphic imbedding $f: \mathbf{B}^{n} \rightarrow D$ satisfying $B_{D}(z, R) \subset f\left(\mathbf{B}^{n}\right)$. Composing with an appropriate automorphism of $\mathbf{B}^{n}$, if necessary, we may assume that $f(0)=z$. For any $\epsilon>0$, there exists $r \in(0,1)$ such that $B_{D}(z, R-\epsilon) \subset f\left(\mathbf{B}^{n}(0, r)\right)$. Since $z^{0} \in \partial D$ is a local holomorphic peak point it follows that (see for example lemma 15.2.2 in 34]) there is a neighbourhood $U_{2} \subset U_{1}$ of $z^{0}$ such that $f\left(\mathbf{B}^{n}(0, r)\right) \subset U \cap D$ whenever $z \in U_{2} \cap D$. Now define $\tilde{f}(z)=f(r z): \mathbf{B}^{n} \rightarrow U \cap D$ which is a biholomorphic imbedding such that $\tilde{f}\left(\mathbf{B}^{n}\right) \supset B_{U \cap D}(z, R-\epsilon)$. Hence $h_{U \cap D}\left(z, \mathbf{B}^{n}\right) \leq 1 /(R-\epsilon)$ whenever 
$z \in U_{2} \cap D$. By taking the infimum over all such $R>0$ we get that $h_{U \cap D}\left(z, \mathbf{B}^{n}\right) \leq h_{D}\left(z, \mathbf{B}^{n}\right)$ whenever $z \in U_{2} \cap D$.

For the lower estimate the following observation will be needed. Fix neighbourhoods $U_{2} \subset U$ of $z^{0}$ as above. Then for every $R>0$ there is a neighbourhood $V \subset U_{2}$ of $z^{0}$ with the property that if $z \in V \cap D$ then $B_{U \cap D}(z, R) \subset U_{2} \cap D$. For this it suffices to show that

$$
\lim _{z \rightarrow z^{0}} d_{U \cap D}\left(z,(U \cap D) \backslash \overline{U_{2} \cap D}\right)=+\infty .
$$

Indeed for every $p \in(U \cap D) \backslash \overline{U_{2} \cap D}$,

$$
d_{U \cap D}(z, p) \geq d_{\Delta}(g(z), g(p)) \rightarrow+\infty
$$

as $z \rightarrow z^{0}$ since $g\left(z^{0}\right)=1$ and $|g|<1$ on $(U \cap D) \backslash \overline{U_{2} \cap D}$. This proves the claim.

Now for a given $R>0$ let $V$ be a sufficiently small neighbourhood of $z^{0}$ so that $B_{U \cap D}(z, R) \subset U_{2} \cap D$ if $z \in V \cap D$. Pick $p \in D$ in the complement of the closure of $B_{U \cap D}(z, R)$ and let $\gamma:[0,1] \rightarrow D$ be a differentiable path with $\gamma(0)=z$ and $\gamma(1)=p$. Then there is a $t_{0} \in(0,1)$ such that $\gamma\left(\left[0, t_{0}\right)\right) \subset$ $B_{U \cap D}(z, R)$ and $\gamma\left(t_{0}\right) \in \partial B_{U \cap D}(z, R)$. Hence

$$
\begin{aligned}
\int_{0}^{1} F_{D}^{K}(\gamma(t), \dot{\gamma}(t)) d t & \geq \int_{0}^{t_{0}} F_{D}^{K}(\gamma(t), \dot{\gamma}(t)) d t \\
& \geq 1 /(1+\epsilon) \int_{0}^{t_{0}} F_{U \cap D}^{K}(\gamma(t), \dot{\gamma}(t)) d t \\
& \geq 1 /(1+\epsilon) d_{U \cap D}\left(z, \gamma\left(t_{0}\right)\right)=R /(1+\epsilon)
\end{aligned}
$$

which implies that $d_{D}(z, p) \geq R /(1+\epsilon)$. In other words,

$$
B_{D}(z, R /(2(1+\epsilon))) \subset B_{U \cap D}(z, R)
$$

if $z \in V \cap D$. To conclude, suppose that there is a biholomorphic imbedding $f: \mathbf{B}^{n} \rightarrow U \cap D$ with $B_{U \cap D}(z, R) \subset f\left(\mathbf{B}^{n}\right)$. It follows from (3.7) that $h_{D}\left(z, \mathbf{B}^{n}\right) \leq 2(1+\epsilon) / R$ or equivalently that

$$
h_{D}\left(z, \mathbf{B}^{n}\right) \leq 2(1+\epsilon) h_{U \cap D}\left(z, \mathbf{B}^{n}\right)
$$

whenever $z \in V \cap D$. Finally, observe that

$$
1 /(2(1+\epsilon)) h_{D}\left(z, \mathbf{B}^{n}\right) \leq h_{U \cap D}\left(z, \mathbf{B}^{n}\right) \leq h_{D}\left(z, \mathbf{B}^{n}\right) \text { for all } z \in V \cap D .
$$

A bounded domain $P \subset \mathbf{C}^{n}$ is an analytic polyhedron if there exist holomorphic functions $f^{1}, \ldots, f^{l}$ defined on an open neighbourhood of the closure of $P$ such that

$$
P=\left\{z \in \mathbf{C}^{n}:\left|f^{1}(z)\right|<1, \ldots,\left|f^{l}(z)\right|<1\right\} .
$$

The generating set $f^{1}, \ldots, f^{l}$ for $P$ will be assumed to be minimal in the sense that none of the $f^{i}$ 's can be dropped without distorting $P$. In addition, if

$$
\left(d f^{i_{1}} \wedge \ldots \wedge d f^{i_{l}}\right) \neq 0 \text { at } p
$$

whenever $\left|f^{i_{1}}(p)\right|=\ldots=\left|f^{i_{k}}(p)\right|=1$ for the unrepeated indices $i_{1}, \ldots, i_{k} \in\{1, \ldots, l\}$, then $P$ is said to be a generic analytic polyhedron.

For an arbitrary bounded domain $\Omega \subset \mathbf{C}^{n}$, let $A(\Omega)$ denote the algebra of those continuous complexvalued functions on $\bar{\Omega}$ which separates the points of $\bar{\Omega}$. A boundary of $\Omega$ for $A(\Omega)$ is a subset $S$ of $\bar{\Omega}$ such that for each $f$ in $A(\Omega)$ there is a point $z$ in $S$ with $|f(z)|=\sup |f|$ on $\bar{\Omega}$. If the class of all boundaries of $\Omega$ contains a smallest set $M$, the set $M$ is called the minimal boundary of $\Omega$. The Shilov boundary $S$ is defined to be the smallest closed boundary. It is already known (see for instance [2]) that such a boundary exists. Moreover, the minimal boundary $M$, if it exists, must be is contained in the Shilov boundary. $S$ is evidently closed. For $P$ a bounded analytic polyhedron as above, there is also a distinguished boundary which consists of those points in $\bar{P}$ at which at least $n$ of the defining functions $f^{i}$ are of modulus 1 . The following theorem in [20] will be useful for our purposes: 
Theorem 3.5. Assume that $P$ is a bounded generic analytic polyhedron as above and let $A(P)$ denote the algebra of continuous functions on $\bar{P}$ which can be uniformly approximated on $\bar{P}$ by functions holomorphic in a neighbourhood of $\bar{P}$. If $l=n$, then the Shilov and the minimal boundaries for $A(P)$ coincide with the distinguished boundary of $P$.

Having identified the Shilov boundary for a generic analytic polyhedron $P$, it is possible to show that the function $h_{P}\left(\cdot, \Delta^{n}\right)$ can be localised near it.

Proposition 3.6. Let $\Omega \subset \mathbf{C}^{n}$ be a domain and $f^{i} \in \mathcal{O}(\Omega)$ for $1 \leq i \leq n$. Let $P$ be a bounded component of $\left\{z \in \Omega:\left|f^{i}(z)\right|<1\right.$ for $\left.1 \leq i \leq n\right\}$. Assume that $P$ is a generic analytic polyhedron in the sense described above. Let $z^{0}$ be a point on the Shilov boundary of $P$, i.e., $\left|f^{i}\left(z^{0}\right)\right|=1$ for $1 \leq i \leq n$. Then for every neighbourhood $U$ of $z^{0}$, there exists a neighbourhood $V \subset U$ of $z^{0}$ such that for all $z \in V \cap P$, we have

$$
c h_{P}\left(z, \Delta^{n}\right) \leq h_{U \cap P}\left(z, \Delta^{n}\right) \leq h_{P}\left(z, \Delta^{n}\right)
$$

where $c>0$ is a constant independent of $z \in V \cap P$ and $\Delta^{n}$ is the unit polydisc in $\mathbf{C}^{n}$.

Proof. Let $U$ be a neighbourhood of $z^{0}$ and let $\theta_{i}, i=1, \ldots, n$ be real numbers satisfying the following properties:

- $F:=\left(e^{\iota \theta_{1}} f^{1}, \ldots, e^{\iota \theta_{n}} f^{n}\right): U \rightarrow \mathbf{C}^{n}$ is a biholomorphic imbedding;

- $F\left(z^{0}\right)=(1, \ldots, 1) \in \mathbf{C}^{n}$

- $F(U \cap P)=F(U) \cap \Delta^{n}$.

This can be achieved by applying the implicit function theorem at the point $z^{0}$. Let $R>0$ such that there exists a biholomorphic imbedding $f: \Delta^{n} \rightarrow P$ satisfying $B_{P}(z, R) \subset f\left(\Delta^{n}\right)$. Composing with an appropriate automorphism of $\Delta^{n}$, if necessary, we may assume that $f(0)=z$. For any $\epsilon>0$, there exists $r \in(0,1)$ such that $B_{P}(z, R-\epsilon) \subset f\left(\Delta^{n}(0, r)\right)$. We claim that there is a neighbourhood $U_{1}$ of $z^{0}$ which is relatively compact in $U$ such that $f\left(\Delta^{n}(0, r)\right) \subset U \cap P$ whenever $z \in U_{1} \cap P$. To see this, suppose the above claim is not true. Then there exist a sequence of points $\left\{w^{k}\right\}_{k=1}^{\infty} \subset \Delta^{n}(0, r)$ and a sequence $\left\{g^{k}\right\}_{k=1}^{\infty}$ of holomorphic mappings $g^{k}: \Delta^{n} \rightarrow P$ such that $g^{k}(0) \rightarrow z^{0}$ as $k \rightarrow \infty$ and

$$
\left|g^{k}\left(w^{k}\right)-z^{0}\right|>\epsilon_{0}
$$

for some $\epsilon_{0}>0$. Since $P$ is bounded, $\left\{g^{k}\right\}$ is a normal family. Let $G$ be any limit of $\left\{g^{k}\right\}$. Then $G(0)=z^{0}$. Consider $\tilde{f}^{i}:=f^{i} \circ G$ for $i=1,2, \ldots, n$. Then $\tilde{f}^{i} \in \mathcal{O}\left(\Delta^{n}\right),\left|\tilde{f}^{i}(0)\right|=1$ for all $1 \leq i \leq n$ and $\left|\tilde{f}^{i}(z)\right| \leq 1$ for all $z \in \Delta^{n}$ and for all $1 \leq i \leq n$. By the maximum modulus theorem $\left|\tilde{f}^{i}\right| \equiv 1$ for all $1 \leq i \leq n$. Hence, $G\left(\Delta^{n}\right) \subset M:=\left\{\left|f^{i}\right|=1,1 \leq i \leq n\right\}$. Since $M$ is a totally real manifold, $G$ is identically a constant. Therefore $G(z) \equiv z^{0}$ since $G(0)=z^{0}$. The constant map $G(z) \equiv z^{0}$ is thus the only limit point of $\left\{g^{k}\right\}$. This contradicts (3.8) and proves the claim.

Moreover, the above argument also shows that for each $\epsilon>0$

$$
F_{P}^{K}(z, v) \leq F_{U \cap P}^{K}(z, v) \leq(1+\epsilon) F_{P}^{K}(z, v)
$$

provided $z$ is close enough to $z^{0}$ and $v$ a tangent vector at $z$.

Hence, there is a neighbourhood $U_{1} \subset U$ of $z^{0}$ such that $f\left(\Delta^{n}(0, r)\right) \subset U \cap P$ whenever $z \in U_{1} \cap P$. It follows that

$$
B_{U \cap P}(z, R-\epsilon) \subset B_{P}(z, R-\epsilon) \subset f\left(\Delta^{n}(0, r)\right)
$$

which implies that

$$
h_{U \cap P}\left(z, \Delta^{n}\right) \leq(R-\epsilon)^{-1}
$$

and hence

for all $z \in U_{1} \cap P$.

$$
h_{U \cap P}\left(z, \Delta^{n}\right) \leq h_{P}\left(z, \Delta^{n}\right)
$$

To establish the lower estimate, observe that for every $R>0$ there is a neighbourhood $V \subset U_{1}$ of $z^{0}$ such that $B_{U \cap P}(z, R) \subset U_{1} \cap P$ whenever $z \in V \cap P$. It suffices to show that $F\left(B_{U \cap P}(z, R)\right) \subset F\left(U_{1} \cap P\right)$ if $z$ is close enough to $z^{0}$. Since biholomorphisms are isometries for the Kobayashi metric, this is the 
same as finding a neighbourhood $V$ of $z^{0}$ such that $B_{F(U \cap P)}(F(z), R) \subset F\left(U_{1}\right) \cap \Delta^{n}$ if $z \in V \cap P$. It is already known that $B_{F(U \cap P)}(w, R) \subset B_{\Delta^{n}}(w, R)$ for all $w \in F(U \cap P)$. We now claim that

$$
B_{\Delta^{n}}(F(z), R) \subset F\left(U_{1}\right) \cap \Delta^{n}
$$

if $z$ is sufficiently close to $z^{0}$. But this is a straight forward consequence of the fact that for any two points $a=\left(a_{1}, a_{2}, \ldots, a_{n}\right)$ and $b=\left(b_{1}, b_{2}, \ldots, b_{n}\right)$ in $\Delta^{n}$,

$$
d_{\Delta^{n}}(a, b)=\max \left(d_{\Delta}\left(a_{1}, b_{1}\right), d_{\Delta}\left(a_{2}, b_{2}\right), \ldots, d_{\Delta}\left(a_{n}, b_{n}\right)\right) .
$$

Now an argument similar to the one in proposition 3.4 that uses (3.9) shows that

$$
h_{P}\left(z, \Delta^{n}\right) \leq 2(1+\epsilon) h_{U \cap P}\left(z, \Delta^{n}\right) .
$$

\section{BeHAVIOUR OF $h$ NEAR STRONGLY PSEUdOCONVEX BOUNDARY POINTS}

We will be using $h_{X}(\cdot)$ to denote $h_{X}\left(\cdot, \mathbf{B}^{n}\right)$ in the sequel unless stated otherwise.

The main purpose of this section is to prove the following which is (i) of theorem 1.1.

Theorem 4.1. Let $D \subset \mathbf{C}^{n}$ be a bounded strongly pseudoconvex domain with $C^{2}$-smooth boundary. Then $h_{D}(z) \rightarrow 0$ as $z \rightarrow \partial D$.

Proof. Let $\left\{z^{k}\right\} \subset D$ be a sequence converging to $z^{0} \in \partial D$. It suffices to show that $h_{D}\left(z^{k}\right) \rightarrow 0$ as $k \rightarrow \infty$. Several lemmas will be needed to complete the proof of this theorem. To start with, the following lemma in [30] will be useful.

Lemma 4.2. let $D$ be a strongly pseudoconvex domain, $\rho$ a defining function for $\partial D$ and $p \in \partial D$. Then there exists a neighbourhood $U$ of $p$ and a family of biholomorphic mappings $h_{\zeta}: \mathbf{C}^{n} \rightarrow \mathbf{C}^{n}$ depending continuously on $\zeta \in \partial D \cap U$ that satisfy the following:

(i) $h_{\zeta}(\zeta)=0$.

(ii) The defining function $\rho_{\zeta}=\rho \circ h_{\zeta}^{-1}$ of the domain $D^{\zeta}:=h_{\zeta}(D)$ has the form

$$
\rho_{\zeta}(z)=2\left(\Re z_{n}+K_{\zeta}(z)\right)+H_{\zeta}(z)+\alpha_{\zeta}(z)
$$

where $K_{\zeta}(z)=\sum_{i, j=1}^{n} a_{i j}(\zeta) z_{i} z_{j}, H_{\zeta}(z)=\sum_{i, j=1}^{n} b_{i j}(\zeta) z_{i} \overline{z_{j}}$ and $\alpha_{\zeta}(z)=o\left(|z|^{2}\right)$ with $K_{\zeta}\left({ }^{\prime} z, 0\right) \equiv 0$ and $\left.\left.H_{\zeta}\left({ }^{\prime} z, 0\right) \equiv\right|^{\prime} z\right|^{2}$.

(iii) The mapping $h_{\zeta}$ takes the real normal to $\partial D$ at $\zeta$ to the real normal $\left\{{ }^{\prime} z=y_{n}=0\right\}$ to $\partial D^{\zeta}$ at the origin.

Here, $z \in \mathbf{C}^{n}$ is written as $z=\left({ }^{\prime} z, z_{n}\right) \in \mathbf{C}^{n-1} \times \mathbf{C}$.

To apply this lemma, select $\zeta^{k} \in \partial D$, closest to $z^{k}$. For $k$ large, the choice of $\zeta^{k}$ is unique since $\partial D$ is sufficiently smooth. Moreover, $\zeta^{k} \rightarrow z^{0}$ and $z^{k} \rightarrow z^{0}$ as $k \rightarrow \infty$. Let $h^{k}:=h_{\zeta^{k}}$ be the biholomorphisms provided by the lemma above. Let $T^{k}: \mathbf{C}^{n} \rightarrow \mathbf{C}^{n}$ be the anisotropic dilation map given by

$$
T^{k}\left({ }^{\prime} z, z_{n}\right)=\left(\frac{{ }^{\prime} z}{\sqrt{\delta_{k}}}, \frac{z_{n}}{\delta_{k}}\right)
$$

and let $D^{k}=T^{k} \circ h^{k}(D)$. We observe that for $k$ large, $h^{k}\left(z^{k}\right)=\left({ }^{\prime} 0,-\delta_{k}\right)$ so that $T^{k} \circ h^{k}\left(z^{k}\right)=\left({ }^{\prime} 0,-1\right)$. Since $h$ is invariant under biholomorphisms, it follows that

$$
h_{D}\left(z^{k}\right)=h_{D^{k}}((' 0,-1))
$$

for all $k$ large. We will show that $h_{D^{k}}\left(\left({ }^{\prime} 0,-1\right)\right) \rightarrow 0$ as $k \rightarrow \infty$.

It has been shown in 30 , that the sequence of domains $\left\{D^{k}\right\}$ converges in the Hausdorff metric to the unbounded realization of the unit ball, namely to

$$
D_{\infty}=\left\{z \in \mathbf{C}^{n}: 2 \Re z_{n}+\left.\left.\right|^{\prime} z\right|^{2}<0\right\} .
$$

It is natural to investigate the behaviour of $d_{D^{k}}(z, \cdot)$ as $k \rightarrow \infty$. To do this, we use ideas from [35].

Lemma 4.3. Let $x^{0} \in D_{\infty}$. Then $d_{D^{k}}\left(x^{0}, \cdot\right) \rightarrow d_{D_{\infty}}\left(x^{0}, \cdot\right)$ uniformly on compact sets of $D_{\infty}$. 
Proof. Let $K \subset D_{\infty}$ be compact and suppose that the desired convergence does not occur. Then there exists a $\epsilon_{0}>0$ and a sequence of points $\left\{p^{k}\right\} \subset K$ which is relatively compact in $D^{k}$ for $k$ large such that

$$
\left|d_{D^{k}}\left(x^{0}, p^{k}\right)-d_{D_{\infty}}\left(x^{0}, p^{k}\right)\right|>\epsilon_{0}
$$

for all $k$ large. By passing to a subsequence, assume that $p^{k} \rightarrow p^{0} \in K$ as $k \rightarrow \infty$. Since $d_{D_{\infty}}\left(x^{0}, \cdot\right)$ is continuous, it follows that

$$
\left|d_{D^{k}}\left(x^{0}, p^{k}\right)-d_{D_{\infty}}\left(x^{0}, p^{0}\right)\right|>\epsilon_{0} / 2
$$

for all $k$ large. Fix $\epsilon>0$ and let $\gamma:[0,1] \rightarrow D_{\infty}$ be a path such that $\gamma(0)=x^{0}, \gamma(1)=p^{0}$ and

$$
\int_{0}^{1} F_{D_{\infty}}^{K}(\gamma(t), \dot{\gamma}(t)) d t<d_{D_{\infty}}\left(x^{0}, p^{0}\right)+\epsilon / 2
$$

Define $\gamma^{k}:[0,1] \rightarrow \mathbf{C}^{n}$ by

$$
\gamma^{k}(t)=\gamma(t)+\left(p^{k}-p^{0}\right) t .
$$

Since the image $\gamma([0,1])$ is compactly contained in $D_{\infty}$ and $p^{k} \rightarrow p^{0} \in K$ as $k \rightarrow \infty$, it follows that $\gamma^{k}:[0,1] \rightarrow D^{k}$ for $k$ large. In addition, $\gamma^{k}(0)=\gamma(0)=x^{0}$ and $\gamma^{k}(1)=\gamma(1)+p^{k}-p^{0}=p^{k}$. It is already known that $F_{D^{k}}^{K}(\cdot, \cdot) \rightarrow F_{D_{\infty}}^{K}(\cdot, \cdot)$ uniformly on compact sets of $D_{\infty} \times \mathbf{C}^{n}$ (see [35]). Also, note that $\gamma^{k} \rightarrow \gamma$ and $\dot{\gamma}^{k} \rightarrow \dot{\gamma}$ uniformly on $[0,1]$. Therefore for $k$ large, we obtain

$$
\int_{0}^{1} F_{D^{k}}^{K}\left(\gamma^{k}(t), \dot{\gamma}^{k}(t)\right) d t \leq \int_{0}^{1} F_{D_{\infty}}^{K}(\gamma(t), \dot{\gamma}(t)) d t+\epsilon / 2<d_{D_{\infty}}\left(x^{0}, p^{0}\right)+\epsilon .
$$

By definition of $d_{D^{k}}\left(x^{0}, p^{k}\right)$ it follows that

$$
d_{D^{k}}\left(x^{0}, p^{k}\right) \leq \int_{0}^{1} F_{D^{k}}^{K}\left(\gamma^{k}(t), \dot{\gamma}^{k}(t)\right) d t \leq d_{D_{\infty}}\left(x^{0}, p^{0}\right)+\epsilon .
$$

Thus

$$
\limsup _{k \rightarrow \infty} d_{D^{k}}\left(x^{0}, p^{k}\right) \leq d_{D_{\infty}}\left(x^{0}, p^{0}\right) .
$$

Conversely, since $K \cup\left\{x^{0}\right\}$ is a compact subset of $D_{\infty}$, it follows that $K \cup\left\{x^{0}\right\}$ is compactly contained $D^{k}$ for all $k$ large. Fix $\epsilon>0$ and let $V \subset U$ be sufficiently small neighbourhoods of $z^{0} \in \partial D$ with $V$ compactly contained in $U$ so that

$$
F_{D}^{K}(z, v) \leq F_{U \cap D}^{K}(z, v) \leq(1+\epsilon) F_{D}^{K}(z, v)
$$

for $z \in V \cap D$ and $v$ a tangent vector at $z$. If $k$ is sufficiently large, $\left(T^{k} \circ h^{k}\right)^{-1}\left(x^{0}\right)$ and $\left(T^{k} \circ h^{k}\right)^{-1}\left(p^{k}\right)$ belong to $V \cap D$. If $U$ is small enough, $U \cap D$ is strictly convex and it follows from Lempert's work [26] that there exist $m_{k}>1$ and holomorphic mappings

$$
\phi^{k}: \Delta\left(0, m_{k}\right) \rightarrow U \cap D
$$

such that $\phi^{k}(0)=\left(T^{k} \circ h^{k}\right)^{-1}\left(x^{0}\right), \phi^{k}(1)=\left(T^{k} \circ h^{k}\right)^{-1}\left(p^{k}\right)$ and

$$
\begin{aligned}
d_{U \cap D}\left(\left(T^{k} \circ h^{k}\right)^{-1}\left(x^{0}\right),\left(T^{k} \circ h^{k}\right)^{-1}\left(p^{k}\right)\right) & =d_{\Delta\left(0, m_{k}\right)}(0,1) \\
& =\int_{0}^{1} F_{U \cap D}^{K}\left(\phi^{k}(t), \dot{\phi}^{k}(t)\right) d t .
\end{aligned}
$$

By [37, it follows that

$$
d_{U \cap D}\left(\left(T^{k} \circ h^{k}\right)^{-1}\left(x^{0}\right),\left(T^{k} \circ h^{k}\right)^{-1}\left(p^{k}\right)\right) \leq(1+\epsilon) d_{D}\left(\left(T^{k} \circ h^{k}\right)^{-1}\left(x^{0}\right),\left(T^{k} \circ h^{k}\right)^{-1}\left(p^{k}\right)\right)
$$

for all $k$ large. Since $T^{k} \circ h^{k}$ are biholomorphisms and hence Kobayashi isometries,

$$
d_{T^{k} \circ h^{k}(U \cap D)}\left(x^{0}, p^{k}\right) \leq(1+\epsilon) d_{D^{k}}\left(x^{0}, p^{k}\right) .
$$


Now (4.4) shows that

$$
\begin{aligned}
\frac{1}{2} \log \left(\frac{m_{k}+1}{m_{k}-1}\right)=d_{\Delta\left(0, m_{k}\right)}(0,1) & =d_{U \cap D}\left(\left(T^{k} \circ h^{k}\right)^{-1}\left(x^{0}\right),\left(T^{k} \circ h^{k}\right)^{-1}\left(p^{k}\right)\right) \\
& =d_{T^{k} \circ h^{k}(U \cap D)}\left(x^{0}, p^{k}\right) \\
& \leq(1+\epsilon) d_{D^{k}}\left(x^{0}, p^{k}\right) .
\end{aligned}
$$

However from (4.2) we have that

$$
d_{D^{k}}\left(x^{0}, p^{k}\right) \leq d_{D_{\infty}}\left(x^{0}, p^{0}\right)+\epsilon<\infty
$$

and hence $m_{k}>1+\delta$ for some uniform $\delta>0$ for all $k$ large. Thus the holomorphic mappings $\sigma^{k}=$ $T^{k} \circ h^{k} \circ \phi^{k}: \Delta(0,1+\delta) \rightarrow T^{k} \circ h^{k}(U \cap D) \subset D^{k}$ are well-defined and satisfy $\sigma^{k}(0)=x^{0}$ and $\sigma^{k}(1)=p^{k}$.

We claim that $\left\{\sigma^{k}\right\}$ admits a subsequence that converges uniformly on compact sets of $\Delta(0,1+\delta)$ to a holomorphic mapping $\sigma: \Delta(0,1+\delta) \rightarrow D_{\infty}$. Indeed consider the disc $\Delta(0, r)$ of radius $r \in(0,1+\delta)$. Observe that $\left(T^{k} \circ h^{k}\right)^{-1} \circ \sigma^{k}(0)=\phi^{k}(0)=\left(T^{k} \circ h^{k}\right)^{-1}\left(x^{0}\right) \rightarrow z^{0} \in \partial D$ as $k \rightarrow \infty$. Let $W$ be a sufficiently small neighbourhood of $z^{0}$. Since $z^{0} \in \partial D$ is a local peak point, it follows that $\left(T^{k} \circ h^{k}\right)^{-1} \circ \sigma^{k}(\Delta(0, r)) \subset$ $W \cap D$ for all $k$ large. If $W$ is small enough, there exists $R>1$ such that for all $k$ large

$$
h^{k}(W \cap D) \subset\left\{z \in \mathbf{C}^{n}:\left|z_{n}+R\right|^{2}+\left.\left.\right|^{\prime} z\right|^{2}<R^{2}\right\} \subset \Omega_{0}
$$

where

$$
\Omega_{0}=\left\{z \in \mathbf{C}^{n}: 2 R\left(\Re z_{n}\right)+\left.\left.\right|^{\prime} z\right|^{2}<0\right\} .
$$

Note that $\Omega_{0}$ is invariant under $T^{k}$ and $\Omega_{0}$ is biholomorphically equivalent to $\mathbf{B}^{n}$. Hence $\sigma^{k}(\Delta(0, r)) \subset$ $T^{k} \circ h^{k}(W \cap D) \subset \Omega_{0}$ for all $k$ large. If $\sigma^{k}(z)=\left({ }^{\prime} \sigma^{k}(z), \sigma_{n}^{k}(z)\right)$ for each $k$, this exactly means that

$$
2 R\left(\Re\left(\sigma_{n}^{k}(z)\right)\right)+\left.\left.\right|^{\prime} \sigma^{k}(z)\right|^{2}<0
$$

whenever $z \in \Delta(0, r)$. It follows that $\left\{\sigma_{n}^{k}(z)\right\}_{k=1}^{\infty}$ and hence each component of $\left\{{ }^{\prime} \sigma^{k}(z)\right\}$ forms a normal family on $\Delta(0, r)$. Since $r \in(0,1+\delta)$ was arbitrary, the usual diagonal subsequence yields a holomorphic mapping $\sigma: \Delta(0,1+\delta) \rightarrow \mathbf{C}^{n}$ or $\sigma \equiv \infty$ on $\Delta(0,1+\delta)$. The latter is not possible since $\sigma(0)=x^{0}$.

It remains to show that $\sigma: \Delta(0,1+\delta) \rightarrow D_{\infty}$. Following [30], note that $D^{k}$ are defined by

$$
\rho^{k}(z)=2 \Re z_{n}+\left.\left.\right|^{\prime} z\right|^{2}+A^{k}(z)
$$

where

$$
\left|A^{k}(z)\right| \leq|z|^{2}\left(c \sqrt{\delta_{k}}+\eta\left(\delta_{k}|z|^{2}\right)\right)
$$

Thus for $z \in \Delta(0, r)$ and $r \in(0,1+\delta)$,

$$
2 R\left(\Re\left(\sigma_{n}^{k}(z)\right)\right)+\left.\left.\right|^{\prime} \sigma^{k}(z)\right|^{2}+A^{k}\left(\sigma^{k}(z)\right)<0
$$

where

$$
\left|A^{k}\left(\sigma^{k}(z)\right)\right|<\left|\sigma^{k}(z)\right|^{2}\left(c \sqrt{\delta_{k}}+\eta\left(\delta_{k}\left|\sigma^{k}(z)\right|^{2}\right)\right) .
$$

Letting $k \rightarrow \infty$ in (4.6) yields

$$
2 R\left(\Re\left(\sigma_{n}^{k}(z)\right)\right)+\left.\left.\right|^{\prime} \sigma^{k}(z)\right|^{2} \leq 0
$$

for $z \in \Delta(0, r)$ or equivalently that $\sigma(\Delta(0, r)) \subset \bar{D}_{\infty}$. Since $r \in(0,1+\delta)$ was arbitrary, it follows that $\sigma(\Delta(0,1+\delta)) \subset \bar{D}_{\infty}$. Since $\sigma(0)=x^{0}$, the maximum principle shows that $\sigma(\Delta(0,1+\delta)) \subset D_{\infty}$. Using (4.3) and (4.4), we get

$$
\begin{aligned}
\int_{0}^{1} F_{D^{k}}^{K}\left(\sigma^{k}(t), \dot{\sigma}^{k}(t)\right) d t & \leq \int_{0}^{1} F_{D}^{K}\left(\phi^{k}(t), \dot{\phi}^{k}(t)\right) d t \\
& \leq \int_{0}^{1} F_{U \cap D}^{K}\left(\phi^{k}(t), \dot{\phi}^{k}(t)\right) d t \\
& =d_{T^{k} \circ h^{k}(U \cap D)}\left(x^{0}, p^{k}\right) \\
& \leq(1+\epsilon) d_{D^{k}}\left(x^{0}, p^{k}\right)
\end{aligned}
$$


Since $\sigma^{k} \rightarrow \sigma$ and $\dot{\sigma}^{k} \rightarrow \dot{\sigma}$ uniformly on $[0,1]$, again exploiting the uniform convergence of $F_{D^{k}}^{K}(\cdot, \cdot) \rightarrow$ $F_{D_{\infty}}^{K}(\cdot, \cdot)$ on compact sets of $D_{\infty} \times \mathbf{C}^{n}$, we see that

$$
\int_{0}^{1} F_{D \infty}^{K}(\sigma(t), \dot{\sigma}(t)) d t \leq \int_{0}^{1} F_{D^{k}}^{K}\left(\sigma^{k}(t), \dot{\sigma}^{k}(t)\right) d t+\epsilon \leq d_{D^{k}}\left(x^{0}, p^{k}\right)+C \epsilon
$$

for all $k$ large. Finally, observe that $\left.\sigma\right|_{[0,1]}$ is a differentiable path in $D_{\infty}$ joining $x^{0}$ and $p^{0}$. Hence by definition

$$
d_{D_{\infty}}\left(x^{0}, p^{0}\right) \leq \int_{0}^{1} F_{D_{\infty}}^{K}(\sigma(t), \dot{\sigma}(t)) d t \leq d_{D^{k}}\left(x^{0}, p^{k}\right)+C \epsilon
$$

Combining (4.2) and (4.7) shows that

$$
\lim _{k \rightarrow \infty} d_{D^{k}}\left(x^{0}, p^{k}\right)=d_{D_{\infty}}\left(x^{0}, p^{0}\right)
$$

which contradicts the assumption (4.1) and proves the required result.

Lemma 4.4. Fix $x^{0} \in D_{\infty}$ and $R>0$. Then

$$
B_{D^{k}}\left(x^{0}, R\right) \rightarrow B_{D_{\infty}}\left(x^{0}, R\right)
$$

in the Hausdorff sense. Moreover, for any $\epsilon>0$ and for all $k$ large

(i) $B_{D_{\infty}}\left(x^{0}, R\right) \subset B_{D^{k}}\left(x^{0}, R+\epsilon\right)$,

(ii) $B_{D^{k}}\left(x^{0}, R-\epsilon\right) \subset B_{D_{\infty}}\left(x^{0}, R\right)$.

Proof. Let $K \subset B_{D_{\infty}}\left(x^{0}, R\right)$ be compact. Then $K$ is a relatively compact subset of $D^{k}$ for all $k$ large and there exists a positive constant $c=c(K) \in(0, R)$ such that $d_{D_{\infty}}\left(x^{0}, z\right)<c$ for all $z \in K$. Pick $\tilde{c} \in(c, R)$. It follows from lemma 4.3 that

$$
d_{D^{k}}\left(x^{0}, z\right) \leq d_{D_{\infty}}\left(x^{0}, z\right)+\tilde{c}-c
$$

for all $z$ in $K$ and for all $k$ large. Therefore

$$
d_{D^{k}}\left(x^{0}, z\right) \leq \tilde{c}<R
$$

for all $z \in K$ and for all $k$ large. This is just the assertion that $K$ is compactly contained in $B_{D^{k}}\left(x^{0}, R\right)$ for all $k$ large. Conversely, let $K \subset \mathbf{C}^{n}$ be a compact set such that $K$ is compactly contained in $B_{D^{k}}\left(x^{0}, R\right)$ for all $k$ large. Then $K$ is relatively compact subset of $D_{\infty}$. Additionally, there exists a positive constant $c=c(K) \in(0, R)$ such that $d_{D^{k}}\left(x^{0}, z\right) \leq c$ for all $z \in K$ and for all $k$ large. Pick $\tilde{c} \in(c, R)$. Again applying lemma 4.3 , we see that

$$
d_{D_{\infty}}\left(x^{0}, z\right)<d_{D^{k}}\left(x^{0}, z\right)+\tilde{c}-c
$$

for all $z \in K$ and all $k$ large. Thus for all $z \in K$, we obtain

$$
d_{D_{\infty}}\left(x^{0}, z\right)<\tilde{c}<R
$$

or equivalently $K$ is compactly contained in $B_{D_{\infty}}\left(x^{0}, R\right)$. This shows that the sequence of domains $\left\{B_{D^{k}}\left(x^{0}, R\right)\right\}$ converges in the Hausdorff metric to $B_{D_{\infty}}\left(x^{0}, R\right)$.

To verify (i), first observe that the closure of $B_{D_{\infty}}\left(x^{0}, R\right)$ is compact since $D_{\infty}$ is Kobayashi complete. Then using lemma 4.3, we get that

$$
d_{D^{k}}\left(x^{0}, z\right) \leq d_{D_{\infty}}\left(x^{0}, z\right)+\epsilon
$$

for all $z$ in the closure of $B_{D_{\infty}}\left(x^{0}, R\right)$ and for all $k$ large. Said differently,

$$
B_{D_{\infty}}\left(x^{0}, R\right) \subset B_{D^{k}}\left(x^{0}, R+\epsilon\right)
$$

for all $k$ large.

For (ii) suppose that the desired result is not true. Then there exists a $\epsilon_{0}>0$ and a sequence of points $\left\{a^{l}\right\}_{l=1}^{\infty} \subset \partial B_{D_{\infty}}\left(x^{0}, R\right)$ such that $a^{l} \in B_{D^{l}}\left(x^{0}, R-\epsilon_{0}\right)$. In view of compactness of $\partial B_{D_{\infty}}\left(x^{0}, R\right)$, we may assume that $a^{l} \rightarrow a \in \partial B_{D_{\infty}}\left(x^{0}, R\right)$ as $l \rightarrow \infty$. It follows from lemma 4.3 that

$$
d_{D^{l}}\left(a^{l}, x^{0}\right) \rightarrow d_{D_{\infty}}\left(a, x^{0}\right)
$$

as $l \rightarrow \infty$. Consequently, $d_{D_{\infty}}\left(a, x^{0}\right) \leq R-\epsilon_{0}$. This violates the fact that $d_{D_{\infty}}\left(a, x^{0}\right)=R$ thereby proving (ii). 
Proof of Theorem 4.1. It suffices to show that $h_{D^{k}}\left(\left({ }^{\prime} 0,-1\right)\right) \rightarrow 0$ as $k \rightarrow \infty$. For any $R>0$, there exists a biholomorphism $\theta: \mathbf{B}^{n} \rightarrow B_{D_{\infty}}\left(\left(^{\prime}(0,-1), R\right)\right.$ as $D_{\infty}$ is biholomorphically equivalent to $\mathbf{B}^{n}$. For $\epsilon>0$ given, there exists a $\delta \in(0,1)$ such that

$$
B_{D_{\infty}}\left(\left({ }^{\prime} 0,-1\right), R-\epsilon\right) \subset \theta\left(\mathbf{B}^{n}(0, \delta)\right) \subset B_{D_{\infty}}\left(\left(^{\prime} 0,-1\right), R\right) .
$$

It follows from lemma 4.4 that

$$
\theta\left(\mathbf{B}^{n}(0, \delta)\right) \subset B_{D^{k}}((' 0,-1), R) \subset D^{k}
$$

and

$$
B_{D^{k}}\left(\left({ }^{\prime} 0,-1\right), R-2 \epsilon\right) \subset B_{D_{\infty}}((' 0,-1), R-\epsilon) .
$$

for all $k$ large. Finally note that (4.8), (4.9) and (4.10) altogether yield that there exists a biholomorphic imbedding $\theta: \mathbf{B}^{n}(0, \delta) \rightarrow D^{k}$ such that

$$
B_{D^{k}}\left(x^{0}, R-2 \epsilon\right) \subset \theta\left(\mathbf{B}^{n}(0, \delta)\right)
$$

so that

$$
h_{D^{k}}\left(\left({ }^{\prime} 0,-1\right)\right) \leq 1 /(R-2 \epsilon) .
$$

Since $R>0$ was arbitrary, we have $h_{D^{k}}\left(\left({ }^{\prime} 0,-1\right)\right) \rightarrow 0$ as $k \rightarrow \infty$. This finishes the proof.

Remark 4.5. Observe that the above result can be stated as

$$
h_{D}(z) \rightarrow h_{D_{\infty}}\left(\left({ }^{\prime} 0,-1\right)\right)=0
$$

as $z \rightarrow z^{0}$ where $D_{\infty} \simeq \mathbf{B}^{n}$ is the model domain at the point $z^{0}$.

Behaviour of $h$ on a strongly pseudoconvex domain $\left(D, c_{D}\right)$ :

In this section, we intend to focus on Fridman's invariant defined using the Carathéodory metric. To be more concrete, let $X$ be a $c$-hyperbolic complex manifold of dimension $n$, i.e., the Carathéodory distance $c_{X}$ is a distance and the topology induced by $c_{X}$ coincides with the Euclidean topology on $X$. Then for $p \in X$

$$
h_{X}\left(p, \mathbf{B}^{n}\right)=\inf _{r \in \mathcal{R}} \frac{1}{r}
$$

where $\mathcal{R}$ denotes the set of all $r>0$ with the property that there is a biholomorphic imbedding $f: \mathbf{B}^{n} \rightarrow$ $X$ with $f\left(\mathbf{B}^{n}\right) \supset B_{X}^{C}(p, r)$, the ball in the Carathéodory metric with radius $r$ around $p \in X$. Evidently, $h_{X}\left(p, \mathbf{B}^{n}\right)$ is a biholomorphic invariant. The notation $h_{X}\left(p, \mathbf{B}^{n}\right)$ is to interpreted in the sense described above for the rest of this section. The following result will be needed for our purposes. The proof is exactly that of proposition 3.3 and is hence omitted.

Proposition 4.6. Let $X$ be a c-hyperbolic manifold of complex dimension $n$. Then

(i) if there is an $x^{0} \in X$ such that $h_{X}\left(x^{0}, \Omega\right)=0$, then $h_{X}\left(x^{0}, \mathbf{B}^{n}\right) \equiv 0$ and $X$ is biholomorphically equivalent to $\mathbf{B}^{n}$.

(ii) $h_{X}\left(\cdot, \mathbf{B}^{n}\right)$ is continuous on $X$.

The goal now will be to investigate the boundary behaviour of $h_{X}\left(\cdot, \mathbf{B}^{n}\right)$ as defined in (4.11) for a strongly pseudoconvex domain $X$. More precisely, the following global statement can be proved:

Theorem 4.7. Let $D \subset \mathbf{C}^{n}$ be a bounded strongly pseudoconvex domain with $C^{2}$-smooth boundary. Then $h_{D}\left(z, \mathbf{B}^{n}\right) \rightarrow 0$ as $z \rightarrow \partial D$.

Proof. Let $\left\{z^{k}\right\} \subset D$ be a sequence converging to $z^{0} \in \partial D$. It suffices to show that $h_{D}\left(z^{k}\right) \rightarrow 0$ as $k \rightarrow \infty$. Choose $\zeta^{k} \in \partial D$, closest to $z^{k}$. Then $\zeta^{k} \rightarrow z^{0}$ as $k \rightarrow \infty$.

We now scale the domain $D$ with respect to the base point $z^{0} \in \partial D$ and the sequence $\left\{\zeta^{k}\right\}$. Let $h^{k}, T^{k}, D^{k}$ and $D_{\infty}$ be defined as before. The first step towards proving theorem 4.7 is to control $c_{D^{k}}(z, \cdot)$ as $k \rightarrow \infty$. To do this, one has to essentially repeat the arguments in 35. We indicate here the necessary changes in [35] to infer stability of the Carathéodory distance. 
Lemma 4.8. For any $x^{0} \in D_{\infty}$

$$
\lim _{k \rightarrow \infty} c_{D^{k}}\left(x^{0}, \cdot\right)=c_{D_{\infty}}\left(x^{0}, \cdot\right)
$$

Moreover, the convergence is uniform on compact sets of $D_{\infty}$.

Proof. Let $K \subset D_{\infty}$ be compact and suppose that the desired convergence does not occur. Then there exists a $\epsilon_{0}>0$ and a sequence of points $\left\{p^{k}\right\} \subset K$ which is relatively compact in $D^{k}$ for $k$ large such that

$$
\left|c_{D^{k}}\left(x^{0}, p^{k}\right)-c_{D_{\infty}}\left(x^{0}, p^{k}\right)\right|>\epsilon_{0}
$$

for all $k$ large. By passing to a subsequence, assume that $p^{k} \rightarrow p^{0} \in K$ as $k \rightarrow \infty$. Since $c_{D_{\infty}}\left(x^{0}, \cdot\right)$ is continuous, it follows that

$$
\left|c_{D^{k}}\left(x^{0}, p^{k}\right)-c_{D_{\infty}}\left(x^{0}, p^{0}\right)\right|>\epsilon_{0} / 2
$$

for all $k$ large.

Let $\phi^{k}: D^{k} \rightarrow \Delta$ be holomorphic maps such that $\phi^{k}\left(x^{0}\right)=0$ and $d_{h y p}\left(0, \phi^{k}\left(p^{k}\right)\right)=c_{D^{k}}\left(x^{0}, p^{k}\right)$. The family $\left\{\phi^{k}\right\}$ is uniformly bounded above. Additionally, since $\left\{D^{k}\right\}$ converges to $D_{\infty}$ in the Hausdorff sense, these maps are defined on an arbitrary compact subset of $D_{\infty}$ and hence some subsequence of $\left\{\phi^{k}\right\}$, which we will be denoting by the same symbols, converges to $\phi: D_{\infty} \rightarrow \bar{\Delta}$. Evidently, $\phi\left(x^{0}\right)=0$. Then the maximum principle forces that $\phi: D_{\infty} \rightarrow \Delta$.

Note that $\phi^{k}\left(p^{k}\right) \rightarrow \phi\left(p^{0}\right)$ as $k \rightarrow \infty$. As a consequence

$$
d_{\text {hyp }}\left(0, \phi^{k}\left(p^{k}\right)\right) \rightarrow d_{\text {hyp }}\left(0, \phi\left(p^{0}\right)\right)
$$

as $k \rightarrow \infty$. Fix $\epsilon>0$ arbitrarily small. Then it follows that

$$
c_{D^{k}}\left(x^{0}, p^{k}\right)=d_{\text {hyp }}\left(0, \phi^{k}\left(p^{k}\right)\right) \leq d_{\text {hyp }}\left(0, \phi\left(p^{0}\right)\right)+\epsilon
$$

for all $k$ large. However from the definition we have that

$$
d_{\text {hyp }}\left(0, \phi\left(p^{0}\right)\right) \leq c_{D_{\infty}}\left(x^{0}, p^{0}\right) .
$$

The above argument shows that

$$
\limsup _{k \rightarrow \infty} c_{D^{k}}\left(x^{0}, p^{k}\right) \leq c_{D_{\infty}}\left(x^{0}, p^{0}\right) .
$$

For the converse, fix $\epsilon>0$ small and note that

$$
c_{D_{\infty}}\left(x^{0}, p^{0}\right) \leq d_{D_{\infty}}\left(x^{0}, p^{0}\right) \leq d_{D^{k}}\left(x^{0}, p^{k}\right)+\epsilon
$$

for all $k$ large. The first inequality is well-known. The second inequality follows from (4.7). Moreover, it is already known that $\left(T^{k} \circ h^{k}\right)^{-1}\left(x^{0}\right)$ and $\left(T^{k} \circ h^{k}\right)^{-1}\left(p^{k}\right)$ both approach $z^{0} \in \partial D$ as $k \rightarrow \infty$ and that

$$
d_{D^{k}}\left(x^{0}, p^{k}\right)=d_{D}\left(\left(T^{k} \circ h^{k}\right)^{-1}\left(x^{0}\right),\left(T^{k} \circ h^{k}\right)^{-1}\left(p^{k}\right)\right)
$$

for all $k$. Let $U$ be a sufficiently small neighbourhood of $z^{0} \in \partial D$. If $U$ is small enough, $U \cap D$ is strictly convex and it follows from Lempert's work 26 that $c_{D}=d_{D}$ on $U \cap D$. It now follows that

$$
\begin{aligned}
d_{D}\left(\left(T^{k} \circ h^{k}\right)^{-1}\left(x^{0}\right),\left(T^{k} \circ h^{k}\right)^{-1}\left(p^{k}\right)\right) & \leq d_{U \cap D}\left(\left(T^{k} \circ h^{k}\right)^{-1}\left(x^{0}\right),\left(T^{k} \circ h^{k}\right)^{-1}\left(p^{k}\right)\right) \\
& =c_{U \cap D}\left(\left(T^{k} \circ h^{k}\right)^{-1}\left(x^{0}\right),\left(T^{k} \circ h^{k}\right)^{-1}\left(p^{k}\right)\right)
\end{aligned}
$$

Applying corollary 10.5.3 of [21, which is essentially a statement about localizing the Carathéodory distance near the boundary, we see that

$$
c_{U \cap D}\left(\left(T^{k} \circ h^{k}\right)^{-1}\left(x^{0}\right),\left(T^{k} \circ h^{k}\right)^{-1}\left(p^{k}\right)\right) \leq(1+\epsilon) c_{D}\left(\left(T^{k} \circ h^{k}\right)^{-1}\left(x^{0}\right),\left(T^{k} \circ h^{k}\right)^{-1}\left(p^{k}\right)\right)
$$

for all $k$ large. Since $T^{k} \circ h^{k}$ are biholomorphisms and hence Kobayashi isometries,

$$
c_{D}\left(\left(T^{k} \circ h^{k}\right)^{-1}\left(x^{0}\right),\left(T^{k} \circ h^{k}\right)^{-1}\left(p^{k}\right)\right)=c_{D^{k}}\left(x^{0}, p^{k}\right) .
$$

Since $\left\{c_{D^{k}}\left(x^{0}, p^{k}\right)\right\}$ is uniformly bounded by (4.13), it follows that

$$
c_{D_{\infty}}\left(x^{0}, p^{0}\right) \leq c_{D^{k}}\left(x^{0}, p^{k}\right)+C \epsilon
$$


for all $k$ large. Combining (4.13) and (4.14), we see that

$$
\lim _{k \rightarrow \infty} c_{D^{k}}\left(x^{0}, p^{k}\right)=c_{D_{\infty}}\left(x^{0}, p^{0}\right) .
$$

This violates (4.12) and hence the result.

Now, an argument similar to one in lemma 4.4 that uses the fact that $\left(D_{\infty}, c_{D_{\infty}}\right)$ is complete and hence closed Carathéodory metric balls are compact shows that

Lemma 4.9. Fix $x^{0} \in D_{\infty}$ and $R>0$. Then $\left\{B_{D^{k}}^{C}\left(x^{0}, R\right)\right\}$ converges to $B_{D_{\infty}}^{C}\left(x^{0}, R\right)$ in the Hausdorff sense. Moreover, for any $\epsilon>0$ and for all $k$ large

(i) $B_{D_{\infty}}^{C}\left(x^{0}, R\right) \subset B_{D^{k}}^{C}\left(x^{0}, R+\epsilon\right)$,

(ii) $B_{D^{k}}^{C}\left(x^{0}, R-\epsilon\right) \subset B_{D_{\infty}}^{C}\left(x^{0}, R\right)$.

Proof of Theorem 4.7 This can be proved by making the relevant changes in the proof of theorem 4.1 using lemmas 4.8 and 4.9 .

\section{Behaviour of $h$ NEAR WEAKLy PSEUdoconvex POINTS OF Finite TYPe IN $\mathbf{C}^{2}$}

The main objective of this section is to establish the following:

Theorem 5.1. Let $D \subset \mathbf{C}^{2}$ be a smoothly bounded weakly pseudoconvex domain of finite type. Let $\left\{p^{j}\right\}$ be a sequence of points in $D$ converging to $p^{0} \in \partial D$. Then

$$
h_{D}\left(p^{j}\right) \rightarrow h_{D_{\infty}}((0,-1))
$$

as $j \rightarrow \infty$ where $D_{\infty}$ is the limiting domain obtained by scaling $D$ with respect to the base point $p^{0}$ and the sequence $\left\{p^{j}\right\}$.

Proof. There are two cases to be considered. After passing to a subsequence if needed,

(i) $\lim _{j \rightarrow \infty} h_{D}\left(p^{j}\right)=0$, or

(ii) $\lim _{j \rightarrow \infty} h_{D}\left(p^{j}\right)>c$ for some positive constant $c$.

In case (i) the domain $D_{\infty}$ will turn out to be biholomorphic to $\mathbf{B}^{n}$ while this will not be the case in (ii).

It will be useful to briefly describe the scaling of $D$ and the corresponding model domain in terms of the base point $p^{0}$ and the sequence $p^{j}$ converging to $p^{0}$. These will require some basic facts about the local geometry of a weakly pseudoconvex domain in $\mathbf{C}^{2}$ near a boundary point of finite type.

\section{Scaling the domain $D$ :}

Let $D \subset \mathbf{C}^{2}$ be a smoothly bounded pseudoconvex domain of finite type defined by $\{\rho(z, \bar{z})=0\}$ for some smooth function $\rho$. We may assume that $p^{0}=(0,0)$ and $\nabla \rho(0,0)=(0,1)$. Then there exists a local coordinate system in a neighbourhood of $(0,0)$ such that the domain $D$ can be written as

$$
\left\{\left(z_{1}, z_{2}\right) \in \mathbf{C}^{2}: 2 \Re z_{2}+H_{2 m}\left(z_{1}, \bar{z}_{1}\right)+o\left(|z|^{2 m}+\Im z_{2}\right)<0\right\}
$$

where $H_{2 m}$ is a homogeneous subharmonic polynomial of degree $2 m \geq 2$ in $z_{1}$ and $\bar{z}_{1}$ which does not contain any harmonic terms. Choose $\zeta^{j} \in \partial D$ defined by

$$
\zeta^{j}=p^{j}+\left(0, \epsilon_{j}\right), \quad \epsilon_{j}>0
$$

From [5] it follows that there exists a sequence $\left\{\phi^{\zeta^{j}}\right\}$ of automorphisms of $\mathbf{C}^{2}$ defined by

$$
\phi^{\zeta^{j}}\left(z_{1}, z_{2}\right)=\left(z_{1}-\zeta_{1}^{j},\left(z_{2}-\zeta_{2}^{j}-\sum_{l=1}^{2 m} d^{l}\left(\zeta^{j}\right)\left(z_{1}-\zeta_{1}^{j}\right)^{l}\right)\left(d^{0}\left(\zeta^{j}\right)\right)^{-1}\right)
$$

where $d^{l}\left(\zeta^{j}\right)$ are non-zero functions depending smoothly on $\zeta^{j}$ and $d^{0}\left(\zeta^{j}\right) \rightarrow 1$ as $j \rightarrow \infty$. Observe that

$$
\phi^{\zeta^{j}}\left(\zeta^{j}\right)=(0,0), \phi^{\zeta^{j}}\left(p^{j}\right)=\left(0,-\epsilon_{j}\left(d^{0}\left(\zeta^{j}\right)\right)^{-1}\right)
$$


and the defining function for $\phi^{\zeta^{j}}(\partial D)$ around the origin is

$$
2 \Re z_{2}+\sum_{l=2}^{2 m} P_{l, \zeta^{j}}\left(z_{1}, \bar{z}_{1}\right)+R_{\zeta^{j}}\left(\Im z_{2}, z_{1}\right)=0
$$

where $P_{l, \zeta^{j}}\left(z_{1}, \bar{z}_{1}\right)$ are real-valued homogeneous polynomials of degree $l$ without any harmonic terms. Also, $P_{l, \zeta^{j}}\left(z_{1}, \bar{z}_{1}\right) \rightarrow 0$ for $l<2 m$ and $P_{2 m, \zeta^{j}}\left(z_{1}, \bar{z}_{1}\right) \rightarrow H_{2 m}\left(z_{1}, \bar{z}_{1}\right)$ as $j \rightarrow \infty$. Let $\|\cdot\|$ be a fixed norm on the finite dimensional space of all real-valued polynomials on the complex plane with degree at most $2 m$ that do not contain any harmonic terms. Define

$$
\tau\left(\zeta^{j}, \epsilon_{j}\right)=\min _{2 \leq l \leq 2 m}\left(\frac{\epsilon_{j}}{\left\|P_{l, \zeta^{j}}\left(z_{1}, \bar{z}_{1}\right)\right\|}\right)^{1 / l} .
$$

Since $P_{2 m, \zeta^{j}} \rightarrow H$ which is a non-zero polynomial, it follows that $\sup _{j}\left(\epsilon_{j}{ }^{-1} \tau\left(\zeta^{j}, \epsilon_{j}\right)^{2 m}\right)<\infty$. Let $\Delta_{\zeta^{j}}^{\epsilon_{j}}: \mathbf{C}^{2} \rightarrow \mathbf{C}^{2}$ be a sequence of dilations defined by

$$
\Delta_{\zeta^{j}}^{\epsilon_{j}}\left(z_{1}, z_{2}\right)=\left(\frac{z_{1}}{\tau\left(\zeta^{j}, \epsilon_{j}\right)}, \frac{z_{2}}{\epsilon_{j}}\right) .
$$

A useful set for approximating the geometry of $D$ near $p^{0}$ is the Catlin's bidisc $Q\left(\zeta^{j}, \epsilon_{j}\right)$ determined by the quantities $\tau\left(\zeta^{j}, \epsilon_{j}\right)$ where

$$
Q\left(\zeta^{j}, \epsilon_{j}\right)=\left(\Delta_{\zeta^{j}}^{\epsilon_{j}} \circ \phi^{\zeta^{j}}\right)^{-1}(\Delta \times \Delta) .
$$

Then the domains $D^{j}=\Delta_{\zeta^{j}}^{\epsilon_{j}} \circ \phi^{\zeta^{j}}(D)$ converge in the Hausdorff metric to

$$
D_{\infty}=\left\{\left(z_{1}, z_{2}\right) \in \mathbf{C}^{2}: 2 \Re z_{2}+P_{\infty}\left(z_{1}, \bar{z}_{1}\right)<0\right\}
$$

where

$$
P_{\infty}\left(z_{1}, \bar{z}_{1}\right)=\lim _{j \rightarrow \infty} \frac{1}{\epsilon_{j}} \sum_{l=2}^{2 m} \tau\left(\zeta^{j}, \epsilon_{j}\right)^{l} P_{l, \zeta^{j}}\left(z_{1}, \bar{z}_{1}\right)
$$

is a real-valued subharmonic polynomial of degree at most $2 m$ without harmonic terms. Note that if the sequence $p^{j}$ converges normally to the point $p^{0}$, i.e., $p^{j}=p^{0}-\epsilon_{j} n\left(p^{0}\right)$ where $n\left(p^{0}\right)$ denotes the unit outward normal to $\partial D$ at $p^{0}$, then it turns out that $P_{\infty}\left(z_{1}, \bar{z}_{1}\right) \equiv H_{2 m}\left(z_{1}, \bar{z}_{1}\right)$. Therefore,

$$
D_{\infty}=\left\{\left(z_{1}, z_{2}\right) \in \mathbf{C}^{2}: 2 \Re z_{2}+H_{2 m}\left(z_{1}, \bar{z}_{1}\right)<0\right\} .
$$

\section{Stability of the infinitesimal Kobayashi metric:}

Lemma 5.2. For $(a, v) \in D_{\infty} \times \mathbf{C}^{n}$,

$$
\lim _{j \rightarrow \infty} F_{D^{j}}^{K}(a, v)=F_{D_{\infty}}^{K}(a, v) .
$$

Moreover, the convergence is uniform on compact sets of $D_{\infty} \times \mathbf{C}^{n}$.

Proof. Let $S \subset D_{\infty}$ and $G \subset \mathbf{C}^{n}$ be compact and suppose that the desired convergence does not occur. Then there is a $\epsilon_{0}>0$ such that after passing to a subsequence, if necessary, we may assume that there exists a sequence of points $\left\{a^{j}\right\} \subset S$ which is relatively compact in $D^{j}$ and a sequence $\left\{v^{j}\right\} \subset G$ such that

$$
\left|F_{D^{j}}^{K}\left(a^{j}, v^{j}\right)-F_{D_{\infty}}^{K}\left(a^{j}, v^{j}\right)\right|>\epsilon_{0}
$$

for $j$ large. Additionally, $a^{j} \rightarrow a \in S$ and $v^{j} \rightarrow v \in G$ as $j \rightarrow \infty$. Since $F_{D_{\infty}}^{K}(a, \cdot)$ is homogeneous, we may assume that $\left|v^{j}\right|=1$ for all $j$. Observe that $D_{\infty}$ is complete hyperbolic and hence taut. The tautness of $D_{\infty}$ implies via a normal family argument that $F_{D_{\infty}}^{K}(\cdot, \cdot)$ is jointly continuous, $0<F_{D_{\infty}}^{K}(a, v)<\infty$ and there exists a holomorphic extremal disc $g: \Delta \rightarrow D_{\infty}$ that by definition satisfies $g(0)=a, g^{\prime}(0)=\mu v$ where $\mu>0$ and $F_{D_{\infty}}^{K}(a, v)=1 / \mu$. Hence

$$
\left|F_{D^{j}}^{K}\left(a^{j}, v^{j}\right)-F_{D_{\infty}}^{K}(a, v)\right|>\epsilon_{0} / 2
$$

for $j$ sufficiently large. Fix $\delta \in(0,1)$ and define the holomorphic mappings $g^{j}: \Delta \rightarrow \mathbf{C}^{2}$ by

$$
g^{j}(z)=g((1-\delta) z)+\left(a^{j}-a\right)+\mu(1-\delta) z\left(v^{j}-v\right) .
$$


Since the image $g((1-\delta) \Delta)$ is compactly contained in $D_{\infty}$ and $a^{j} \rightarrow a, v^{j} \rightarrow v$ as $j \rightarrow \infty$, it follows that $g^{j}: \Delta \rightarrow D^{j}$ for $j$ large. Also, $g^{j}(0)=g(0)+a^{j}-a=a^{j}$ and $\left(g^{j}\right)^{\prime}(0)=(1-\delta) g^{\prime}(0)+\mu(1-\delta)\left(v^{j}-v\right)=$ $\mu(1-\delta) v^{j}$. By the definition of the infinitesimal metric it follows that

$$
F_{D^{j}}^{K}\left(a^{j}, v^{j}\right) \leq \frac{1}{\mu(1-\delta)}=\frac{F_{D_{\infty}}^{K}(a, v)}{\mu(1-\delta)} .
$$

Letting $\delta \rightarrow 0^{+}$yields

$$
\limsup _{j \rightarrow \infty} F_{D^{j}}^{K}\left(a^{j}, v^{j}\right) \leq F_{D_{\infty}}^{K}(a, v) .
$$

Conversely, fix $\epsilon>0$ arbitrarily small. By definition, there are holomorphic mappings $f^{j}: \Delta \rightarrow D^{j}$ satisfying $f^{j}(0)=a^{j}$ and $\left(f^{j}\right)^{\prime}(0)=\mu^{j}$ where $\mu^{j}>0$ and

$$
F_{D^{j}}^{K}\left(a^{j}, v^{j}\right) \geq \frac{1}{\mu^{j}}-\epsilon
$$

The sequence $\left\{f^{j}\right\}$ has a subsequence that converges to a holomorphic mapping $f: \Delta \rightarrow D_{\infty}$ uniformly on compact sets of $\Delta$. To see this, consider $\Delta(0, r)$ for $r \in(0,1)$. We may assume that $S$ is compactly contained in $\Delta\left(0, C_{1}^{1 / 2 m}\right) \times \Delta\left(0, C_{1}\right)$ for some $C_{1}>1$. As a consequence

$$
a^{j} \in \Delta\left(0, C_{1}^{1 / 2 m}\right) \times \Delta\left(0, C_{1}\right) \subset \Delta\left(0, \frac{\tau\left(\zeta^{j}, C_{1} \epsilon_{j}\right)}{\tau\left(\zeta^{j}, \epsilon_{j}\right)}\right) \times \Delta\left(0, \frac{C_{1} \epsilon_{j}}{\epsilon_{j}}\right)
$$

for all $j$. In particular, for all $j$

$$
\left(\Delta_{\zeta^{j}}^{\epsilon_{j}} \circ \phi^{\zeta^{j}}\right)^{-1}\left(a^{j}\right) \in Q\left(\zeta^{j}, C_{1} \epsilon_{j}\right)
$$

Also, note that

$$
\left(\Delta_{\zeta^{j}}^{\epsilon_{j}} \circ \phi^{\zeta^{j}}\right)^{-1}\left(a^{j}\right) \rightarrow p^{0} \in \partial D
$$

as $j \rightarrow \infty$. Now, applying proposition 1 in 4 to the mappings

$$
\left(\Delta_{\zeta^{j}}^{\epsilon_{j}} \circ \phi^{\zeta^{j}}\right)^{-1} \circ f^{j}: \Delta \rightarrow D
$$

shows that there exists a uniform positive constant $C_{2}=C_{2}(r)$ with the property that

$$
\left(\Delta_{\zeta^{j}}^{\epsilon_{j}} \circ \phi^{\zeta^{j}}\right)^{-1} \circ f^{j}(\Delta(0, r)) \subset Q\left(\zeta^{j}, C_{2} C_{1} \epsilon_{j}\right)
$$

or equivalently that

$$
f^{j}(\Delta(0, r)) \subset \Delta\left(0, \sqrt{C_{1} C_{2}}\right) \times \Delta\left(0, C_{1} C_{2}\right) .
$$

Therefore, $\left\{f^{j}\right\}$ is a normal family. Hence, the sequence $\left\{f^{j}\right\}$ has a subsequence that converges uniformly on compact sets of $\Delta$ to a holomorphic mapping $f: \Delta \rightarrow \mathbf{C}^{2}$ or $f \equiv \infty$. The latter cannot be true since $f(0)=a$. It remains to show that $f: \Delta \rightarrow D_{\infty}$. For this note that $D^{j}$ are defined by

$$
2 \epsilon_{j} \Re z_{2}+\sum_{l=2}^{m} \tau\left(\zeta^{j}, \epsilon_{j}\right)^{l} P_{l, \zeta^{j}}\left(z_{1}, \bar{z}_{1}\right)+R_{\zeta^{j}}\left(\epsilon_{j} \Im z_{2}, \tau\left(\zeta^{j}, \epsilon_{j}\right) z_{1}\right)<0
$$

where $R_{\zeta^{j}}\left(\epsilon_{j} \Im z_{2}, \tau\left(\zeta^{j}, \epsilon_{j}\right) z_{1}\right)=\epsilon_{j} o(1)$ and the term $o(1)$ is uniformly convergent to zero as $j \rightarrow \infty$. Thus, for $w \in \Delta(0, r)$ and $r \in(0,1)$

$$
2 \epsilon_{j} \Re\left(f_{2}^{j}(w)\right)+\sum_{l=2}^{m} \tau\left(\zeta^{j}, \epsilon_{j}\right)^{l} P_{l, \zeta^{j}}\left(f_{1}^{j}(w), \overline{f_{1}^{j}(w)}\right)+R_{\zeta^{j}}\left(\epsilon_{j} \Im\left(f_{2}^{j}(w)\right), \tau\left(\zeta^{j}, \epsilon_{j}\right) f_{1}^{j}(w)\right)<0 .
$$

Letting $j \rightarrow \infty$ yields

$$
2 \Re\left(f_{2}(w)\right)+P_{\infty}\left(f_{1}(w), \overline{f_{1}(w)}\right) \leq 0
$$

or equivalently that $f(\Delta(0, r)) \subset \bar{D}_{\infty}$. Since $r \in(0,1)$ was arbitrary, it follows that $f(\Delta) \subset \bar{D}_{\infty}$. Since $f(0,0)=a$ the maximum principle forces that $f: \Delta \rightarrow D_{\infty}$. Note that

$$
f^{\prime}(0)=\lim _{j \rightarrow \infty}\left(f^{j}\right)^{\prime}(0)=\lim _{j \rightarrow \infty} \mu^{j} v^{j}=\mu v
$$

for some $\mu>0$. It follows from the definition of the infinitesimal metric that

$$
F_{D_{\infty}}^{K}(a, v) \leq 1 / \mu .
$$


The above observation together with (5.3) yields

$$
\liminf _{j \rightarrow \infty} F_{D^{j}}^{K}\left(a^{j}, v^{j}\right) \geq F_{D_{\infty}}^{K}(a, v) .
$$

Combining (5.2) and (5.4) shows that

$$
\lim _{j \rightarrow \infty} F_{D^{j}}^{K}\left(a^{j}, v^{j}\right)=F_{D_{\infty}}^{K}(a, v)
$$

which contradicts the assumption (5.1) and proves the lemma.

Remark 5.3. This lemma does not directly follow from [41] since there is no taut domain that contains all the scaled domains $D^{j}$.

Write $(0,-1)=z^{0}$ and $\left(0,-1 / d^{0}\left(\zeta^{j}\right)\right)=z^{j}$ for brevity.

Proof of Theorem 5.1(i): For each $j$, let $1 / R_{j}$ be a positive number that almost realizes $h_{D}\left(p^{j}\right)$, i.e., $1 / R_{j}<h_{D}\left(p^{j}\right)+\epsilon$ for some fixed $\epsilon>0$. Evidently, the sequence $R_{j} \rightarrow \infty$ and there exists a sequence of biholomorphic imbeddings $F^{j}: \mathbf{B}^{2} \rightarrow D$ satisfying $F^{j}(0)=p^{j}$ and $B_{D}\left(p^{j}, R_{j}\right) \subset F^{j}\left(\mathbf{B}^{2}\right)$. Consider the dilated maps

$$
\psi^{j}:=\Delta_{\zeta^{j}}^{\epsilon_{j}} \circ \phi^{\zeta^{j}} \circ F^{j}: \mathbf{B}^{2} \rightarrow D^{j} .
$$

Note that $\Delta_{\zeta^{j}}^{\epsilon_{j}} \circ \phi^{\zeta^{j}} \circ F^{j}(0,0)=\left(0,-1 / d^{0}\left(\zeta^{j}\right)\right) \rightarrow(0,-1)$ as $j \rightarrow \infty$. In this setting, theorem 2 of [4] (see proposition 2.2 in [3] also) shows that the sequence $\left\{\Delta_{\zeta^{j}}^{\epsilon_{j}} \circ \phi^{\zeta^{j}} \circ F^{j}\right\}$ admits a subsequence that will still be denoted by the same indices, that converges uniformly on compact sets of $\mathbf{B}^{2}$ to a holomorphic mapping $\psi: \mathbf{B}^{2} \rightarrow \mathbf{C}^{2}$. Now an argument similar to the one in the proof of lemma 5.2 shows that $\psi: \mathbf{B}^{2} \rightarrow D_{\infty}$. Then $\psi$ is a biholomorphism. To establish this, it will suffice to show that for each $\epsilon>0$,

$$
B_{D_{\infty}}\left(z^{0}, R-\epsilon\right) \subset B_{D^{j}}\left(z^{j}, R\right)
$$

for all $R>0$ and all $j$ large and this will follow from

$$
\limsup _{j \rightarrow \infty} d_{D^{j}}\left(z^{j}, \cdot\right) \leq d_{D_{\infty}}\left(z^{0}, \cdot\right) .
$$

For this fix $q \in D_{\infty}$ and let $\gamma:[0,1] \rightarrow D_{\infty}$ be a piecewise $C^{1}$-smooth path in $D_{\infty}$ such that $\gamma(0)=$ $z^{0}, \gamma(1)=q$ and

Define $\gamma^{j}:[0,1] \rightarrow \mathbf{C}^{2}$ by

$$
\int_{0}^{1} F_{D_{\infty}}^{K}(\gamma(t), \dot{\gamma}(t)) d t \leq d_{D_{\infty}}\left(z^{0}, q\right)+\epsilon / 2
$$

$$
\gamma^{j}(t)=\gamma(t)+\left(z^{j}-z^{0}\right)(1-t) .
$$

Since the trace of $\gamma$ is relatively compact in $D_{\infty}$ and $z^{j} \rightarrow z^{0}$, it follows that the trace of $\gamma$ is contained uniformly relatively compactly in $D^{j}$ for all large $j$. Note that $\gamma^{j}(0)=\gamma(0)+z^{j}-z^{0}=z^{j}$ and $\gamma^{j}(1)=q$. In addition, $\gamma^{j} \rightarrow \gamma$ and $\dot{\gamma}^{j} \rightarrow \dot{\gamma}$ uniformly on $[0,1]$. It follows from lemma 5.2 that

$$
\int_{0}^{1} F_{D^{j}}^{K}\left(\gamma^{j}(t), \dot{\gamma}^{j}(t)\right) d t \leq \int_{0}^{1} F_{D_{\infty}}^{K}(\gamma(t), \dot{\gamma}(t)) d t+\epsilon / 2 \leq d_{D_{\infty}}\left(z^{0}, q\right)+\epsilon .
$$

Consequently,

which implies that

$$
d_{D^{j}}\left(z^{j}, q\right) \leq \int_{0}^{1} F_{D^{j}}^{K}\left(\gamma^{j}(t), \dot{\gamma}^{j}(t)\right) d t \leq d_{D_{\infty}}\left(z^{0}, q\right)+\epsilon
$$

$$
\limsup _{j \rightarrow \infty} d_{D^{j}}\left(z^{j}, \cdot\right) \leq d_{D_{\infty}}\left(z^{0}, \cdot\right) .
$$

Note that $B_{D}\left(p^{j}, R_{j}\right) \subset F^{j}\left(\mathbf{B}^{2}\right)$. Since $\Delta_{\zeta^{j}}^{\epsilon_{j}} \circ \phi^{\zeta^{j}}$ are biholomorphisms and hence Kobayashi isometries, it follows that

$$
B_{D^{j}}\left(z^{j}, R_{j}\right) \subset \Delta_{\zeta^{j}}^{\epsilon_{j}} \circ \phi^{\zeta^{j}} \circ F^{j}\left(\mathbf{B}^{2}\right) .
$$

Since $\left(D_{\infty}, d_{D_{\infty}}\right)$ is complete, it is possible to write

$$
D_{\infty}=\bigcup_{\nu=1}^{\infty} B_{D_{\infty}}((0,-1), \nu)
$$


which is an exhaustion of $D_{\infty}$ by an increasing union of relatively compact domains. Consider

$$
\theta^{j}:=\left(\Delta_{\zeta^{j}}^{\epsilon_{j}} \circ \phi^{\zeta^{j}} \circ F^{j}\right)^{-1}: \Delta_{\zeta^{j}}^{\epsilon_{j}} \circ \phi^{\zeta^{j}} \circ F^{j}\left(\mathbf{B}^{2}\right) \rightarrow \mathbf{B}^{2} .
$$

These mappings are evidently defined on an arbitrary compact subset of $D_{\infty}$ for large $j$ and hence some subsequence of $\left\{\theta^{j}\right\}$ converges to $\theta: D_{\infty} \rightarrow \overline{\mathbf{B}}^{2}$. Moreover, $\theta(0,-1)=(0,0)$ together with the maximum principle shows that $\theta: D_{\infty} \rightarrow \mathbf{B}^{2}$. Finally observe that for $w$ in a fixed compact set in $D_{\infty}$,

$$
\begin{aligned}
|\psi \circ \theta(w)-w| & =\left|\psi \circ \theta(w)-\psi^{j} \circ \theta^{j}(w)\right| \\
& =\left|\psi \circ \theta(w)-\psi \circ \theta^{j}(w)\right|+\left|\psi \circ \theta^{j}(w)-\psi^{j} \circ \theta^{j}\right| \\
& \rightarrow 0 \text { as } j \rightarrow \infty
\end{aligned}
$$

This shows that $\psi \circ \theta=i d$. Similarly, it can be proved that $\theta \circ \psi=i d$. This shows that $D_{\infty}$ is biholomorphically equivalent to $\mathbf{B}^{2}$. In particular, $h_{D_{\infty}}(\cdot) \equiv 0$ so that $h_{D}\left(p^{j}\right) \rightarrow h_{D_{\infty}}((0,-1))$ as $j \rightarrow \infty$. This completes the proof of case (i).

Case (ii) differs from (i) in one important way. To prove that the integrated Kobayashi distance is stable under scaling in the strongly pseodoconvex case, Lempert's theorem that guarantees the existence of complex geodesics in strongly convex domains was used. This approach will evidently not work for weakly pseudoconvex domains. To control the integrated Kobayashi distance in weakly pseudoconvex domain under scaling, the following two ingredients will be required that serve to circumvent the need for complex geodesics.

Lemma 5.4. Let $D$ be a Kobayashi hyperbolic domain in $\mathbf{C}^{n}$ with a subdomain $D^{\prime} \subset D$. Let $p, q \in D^{\prime}$, $d_{D}(p, q)=a$ and $b>a$. If $D^{\prime}$ satisfies the condition $B_{D}(q, b) \subset D^{\prime}$, then the following two inequalities hold:

$$
\begin{aligned}
d_{D^{\prime}}(p, q) & \leq \frac{1}{\tanh (b-a)} d_{D}(p, q), \\
F_{D^{\prime}}^{K}(p, v) & \leq \frac{1}{\tanh (b-a)} F_{D}^{K}(p, v) .
\end{aligned}
$$

The reader is referred to [22] (or [23]) for a proof, but it should be noted that this statement emphasizes an upper bound for $d_{D^{\prime}}$ in terms of $d_{D}$. An estimate with the inequality reversed is an immediate consequence of the definition of the Kobayashi metric.

The second ingredient is an estimate for the Kobayashi metric between two points in a weakly pseudoconvex finite type domain $D$ in $\mathbf{C}^{2}$ due to Herbort $([19])$. To state this, let $d(\cdot, \partial D)$ be the Euclidean distance to the boundary and $\rho$ a smooth defining function for $\partial D$. For $a, b \in D$, define

$$
\begin{aligned}
\rho^{*}(a, b) & =\log \left(1+\frac{d(a, b)}{d(a, \partial D)}+\frac{|\langle L(a), a-b\rangle|}{\tau(a, d(a, \partial D))}\right) \\
L(a) & =\left(-\frac{\partial \rho}{\partial z_{2}}(a), \frac{\partial \rho}{\partial z_{1}}(a)\right) \\
d(a, b) & =\min \left\{d^{\prime}(a, b),|a-b|\right\} \\
d^{\prime}(a, b) & =\inf \{\delta>0: a \in Q(b, \delta)\},
\end{aligned}
$$

where $\langle\cdot, \cdot\rangle$ denotes the standard hermitian inner product in $\mathbf{C}^{2}$.

The main result of [19] that is needed is:

Theorem 5.5. Assume that $D=\{\rho<0\} \subset \mathbf{C}^{2}$ be a bounded pseudoconvex domain with smooth boundary such that all boundary points are of finite type. Then there exists a positive constant $C_{*}$ such that for any two points $a, b \in D$

$$
C_{*}\left(\rho^{*}(a, b)+\rho^{*}(b, a)\right) \leq d_{D}(a, b) \leq 1 / C_{*}\left(\rho^{*}(a, b)+\rho^{*}(b, a)\right) .
$$

Observe that in case (ii) the largest radii admissible in the definition of the Fridman's invariant function $h_{D}\left(p^{j}\right)$ is at most $\nu_{0}$ where $\nu_{0}=1 / c$. Several lemmas will be needed to complete the proof in this case. We first note the following: 
Lemma 5.6. For all $R>0$ and for all $j$ large, $B_{D^{j}}\left(z^{j}, R\right)$ is compactly contained in $D_{\infty}$.

Proof. The proof divides into two parts. In the first part we show that the sets $B_{D^{j}}\left(z^{j}, R\right)$ cannot accumulate at the point at infinity in $\partial D_{\infty}$ and in the second part we show that the sets $B_{D^{j}}\left(z^{j}, R\right)$ do not cluster at any finite boundary point. First note that

$$
B_{D^{j}}\left(z^{j}, R\right)=\Delta_{\zeta^{j}}^{\epsilon_{j}} \circ \phi^{\zeta^{j}}\left(B_{D}\left(p^{j}, R\right)\right)
$$

Assume that $q \in B_{D}\left(p^{j}, R\right)$. Using Herbort's lower estimate for the Kobayashi metric gives us

$$
C_{*}\left(\rho^{*}\left(p^{j}, q\right)+\rho^{*}\left(q, p^{j}\right)\right) \leq d_{D}\left(p^{j}, q\right) .
$$

As a consequence

$$
d\left(p^{j}, q\right)<\exp \left(R / C_{*}\right) d\left(p^{j}, \partial D\right)
$$

which in turn implies that

- either $\left|p^{j}-q\right|<d\left(p^{j}, \partial D\right) \exp \left(R / C_{*}\right)$ or

- for each $j$, there exists a $\delta_{j} \in\left(0, d\left(p^{j}, \partial D\right) \exp \left(R / C_{*}\right)\right)$ such that $p^{j} \in Q\left(q, \delta_{j}\right)$.

It follows from proposition 1.7 in [5] that there exists a uniform positive constant $C$ such that for each $j$, the following holds: if $p^{j} \in Q\left(q, \delta_{j}\right)$, then $q \in Q\left(p^{j}, C \delta_{j}\right)$. Hence, the second statement above can be rewritten as: there exists a positive constant $C$ such that for each $j$, there exists a $\delta_{j} \in\left(0, d\left(p^{j}, \partial D\right) \exp \left(R / C_{*}\right)\right)$ with the property that

$$
q \in\left(\phi^{p^{j}}\right)^{-1}\left(\Delta\left(0, \tau\left(p^{j}, C \delta_{j}\right)\right) \times \Delta\left(0, C \delta_{j}\right)\right) .
$$

Said differently, $B_{D^{j}}\left(p^{j}, R\right)$ is contained in the union

$$
B_{D^{j}}\left(p^{j}, R\right) \subset B\left(p^{j}, d\left(p^{j}, \partial D\right) \exp \left(R / C_{*}\right)\right) \cup\left(\phi^{p^{j}}\right)^{-1}\left(\Delta\left(0, \tau\left(p^{j}, C \delta_{j}\right)\right) \times \Delta\left(0, C \delta_{j}\right)\right)
$$

with $\delta_{j}$ as described above. Now, using the explicit expression for $\phi^{\zeta^{j}}$ we get

$$
\begin{gathered}
\phi^{\zeta^{j}}\left\{\left(z_{1}, z_{2}\right) \in \mathbf{C}^{2}:\left|z_{1}-p_{1}^{j}\right|^{2}+\left|z_{2}-p_{2}^{j}\right|^{2}<\left(d\left(p^{j}, \partial D\right)\right)^{2} \exp \left(2 R / C_{*}\right)\right\}= \\
\left\{\left(w_{1}, w_{2}\right):\left|w_{1}\right|^{2}+\left|d^{0}\left(\zeta^{j}\right) w_{2}+\epsilon_{j}+\sum_{l=1}^{2 m} d^{l}\left(\zeta^{j}\right) w_{1}^{l}\right|^{2}<\left(d\left(p^{j}, \partial D\right)\right)^{2} \exp \left(2 R / C_{*}\right)\right\} .
\end{gathered}
$$

Hence

$$
\begin{gathered}
\Delta_{\zeta^{j}}^{\epsilon_{j}} \circ \phi^{\zeta^{j}}\left\{\left(z_{1}, z_{2}\right) \in \mathbf{C}^{2}:\left|z_{1}-p_{1}^{j}\right|^{2}+\left|z_{2}-p_{2}^{j}\right|^{2}<\left(d\left(p^{j}, \partial D\right)\right)^{2} \exp \left(2 R / C_{*}\right)\right\}= \\
\left\{w:\left|w_{1}\right|^{2}+\left(\frac{\epsilon_{j}}{\tau\left(\zeta^{j}, \epsilon_{j}\right)}\right)^{2}\left|d^{0}\left(\zeta^{j}\right) w_{2}+1+\epsilon_{j}^{-1}\left(\sum_{l=1}^{2 m} \alpha^{j, l} w_{1}^{l}\right)\right|^{2}<\frac{\left(d\left(p^{j}, \partial D\right)\right)^{2} \exp \left(2 R / C_{*}\right)}{\tau\left(\zeta^{j}, \epsilon_{j}\right)^{2}}\right\}
\end{gathered}
$$

where

$$
\alpha^{j, l}=d^{l}\left(\zeta^{j}\right) \tau\left(\zeta^{j}, \epsilon_{j}\right)^{l} .
$$

If $w=\left(w_{1}, w_{2}\right)$ belongs to the set described by (5.6) above, then

$$
\begin{aligned}
\left|w_{1}\right| & \leq \frac{d\left(p^{j}, \partial D\right) \exp \left(R / C_{*}\right)}{\tau\left(\zeta^{j}, \epsilon_{j}\right)} \lesssim \frac{\epsilon_{j} \exp \left(R / C_{*}\right)}{\tau\left(\zeta^{j}, \epsilon_{j}\right)} \quad \text { and } \\
\left|d^{0}\left(\zeta^{j}\right) w_{2}+1+\epsilon_{j}{ }^{-1}\left(\sum_{l=1}^{2 m} \alpha^{j, l} w_{1}^{l}\right)\right| & \leq \frac{d\left(p^{j}, \partial D\right) \exp \left(R / C_{*}\right)}{\epsilon_{j}} \lesssim \exp \left(R / C_{*}\right) .
\end{aligned}
$$

Moreover, for $\delta_{j} \in\left(0, d\left(p^{j}, \partial D\right) \exp \left(R / C_{*}\right)\right)$,

$$
\begin{gathered}
\left(\phi^{p^{j}}\right)^{-1}\left\{\left(z_{1}, z_{2}\right) \in \mathbf{C}^{2}:\left|z_{1}\right|<\tau\left(p^{j}, C \delta_{j}\right),\left|z_{2}\right|<C \delta_{j}\right\}= \\
\left\{\left(w_{1}, w_{2}\right):\left|w_{1}-p_{1}^{j}\right|<\tau\left(p^{j}, C \delta_{j}\right),\left|w_{2}-p_{2}^{j}-\sum_{l=1}^{2 m} d^{l}\left(p^{j}\right)\left(w_{1}-p_{1}^{j}\right)^{l}\right|<C \delta_{j} d^{0}\left(p^{j}\right)\right\}
\end{gathered}
$$


so that

$$
\left\{w:\left|w_{1}\right|<\frac{\tau\left(p^{j}, C \delta_{j}\right)}{\tau\left(\zeta^{j}, \epsilon_{j}\right)},\left|d^{0}\left(\zeta^{j}\right) w_{2}+1+\epsilon_{j}^{-1}\left(\sum_{l=1}^{2 m} \beta^{j, l} w_{1}^{l}\right)\right|<\frac{C \delta_{j} d^{0}\left(p^{j}\right)}{\epsilon_{j}}\right\}
$$

where

$$
\beta^{j, l}=\left(d^{l}\left(\zeta^{j}\right)-d^{l}\left(p^{j}\right)\right) \tau\left(\zeta^{j}, \epsilon_{j}\right)^{l} .
$$

If $w=\left(w_{1}, w_{2}\right)$ belongs to the set given by (5.9), then

$$
\begin{aligned}
\left|w_{1}\right| & <\frac{\tau\left(p^{j}, C \delta_{j}\right)}{\tau\left(\zeta^{j}, \epsilon_{j}\right)} \quad \text { and } \\
\left|d^{0}\left(\zeta^{j}\right) w_{2}+1+\epsilon_{j}{ }^{-1}\left(\sum_{l=1}^{2 m} \beta^{j, l} w_{1}^{l}\right)\right| & <\frac{C \delta_{j} d^{0}\left(p^{j}\right)}{\epsilon_{j}} \\
& <\frac{C d\left(p^{j}, \partial D\right) \exp \left(R / C_{*}\right) d^{0}\left(p^{j}\right)}{\epsilon_{j}} \\
& \lesssim \exp \left(R / C_{*}\right) d^{0}\left(p^{j}\right) .
\end{aligned}
$$

It follows from Catlin's work that

- $\epsilon_{j}{ }^{1 / 2} \lesssim \tau\left(\zeta^{j}, \epsilon_{j}\right) \lesssim \epsilon_{j}{ }^{1 / 2 m}$

- $\tau\left(p^{j}, C \epsilon_{j}\right) \approx \tau\left(\zeta^{j}, \epsilon_{j}\right)$

- $\left|d^{l}\left(\zeta^{j}\right)\right| \lesssim \epsilon_{j}\left(\tau\left(\zeta^{j}, \epsilon_{j}\right)\right)^{-l}$ for all $1 \leq l \leq 2 m$

- $\left|d^{l}\left(p^{j}\right)\right| \lesssim \epsilon_{j}\left(\tau\left(p^{j}, \epsilon_{j}\right)\right)^{-l}$ for all $1 \leq l \leq 2 m$

- $d^{0}\left(\zeta^{j}\right) \approx 1$ and $d^{0}\left(p^{j}\right) \approx 1$.

These estimates together with (5.7), (5.8), (5.10) and (5.11) show that if $w=\left(w_{1}, w_{2}\right)$ belongs either of (5.6) or (5.9), then $|w|$ is uniformly bounded. In other words, the sets

$$
\Delta_{\zeta^{j}}^{\epsilon_{j}} \circ \phi^{\zeta^{j}}\left(B\left(p^{j}, d\left(p^{j}, \partial D\right) \exp \left(R / C_{*}\right)\right)\right) \bigcup \Delta_{\zeta^{j}}^{\epsilon_{j}} \circ \phi^{\zeta^{j}}\left(\left(\phi^{p^{j}}\right)^{-1}\left(\Delta\left(0, \tau\left(p^{j}, C \delta_{j}\right)\right) \times \Delta\left(0, C \delta_{j}\right)\right)\right)
$$

are uniformly bounded. Therefore, $B_{D^{j}}\left(z^{j}, R\right)$ as a set cannot cluster at the point at infinity on $\partial D_{\infty}$.

It remains to show that $B_{D^{j}}\left(z^{j}, R\right)$ do not cluster on the finite part of $\partial D$. Suppose there exists a sequence of points $\left\{q^{j}\right\}, q^{j} \in B_{D^{j}}\left(z^{j}, R\right)$ such that $q^{j} \rightarrow q^{0}$ as $j \rightarrow \infty$ for some $q^{0}$ a finite boundary point of $D_{\infty}$. Then proposition 4.1 of $\left[9\right.$ shows that there exists a neighbourhood $V$ of $q^{0}$ in $\mathbf{C}^{2}$ and a uniform positive constant $C$ such that for all $z \in V \cap D^{j}$ and $v$ a tangent vector at $z$,

$$
F_{D^{j}}^{K}(z, v) \geq C \frac{|v|}{d\left(z, \partial D^{j}\right)^{1 / 2 m}}
$$

for all $j$ large. Choose a neighbourhood $\tilde{V}$ of $z^{0}$ which is compactly contained in $D$ and disjoint from $V$. We may assume that $\left\{z^{j}\right\} \subset \tilde{V}$ for all $j$ large. Let $\gamma^{j}$ be a arbitrary piecewise $C^{1}$ curve in $D^{j}$ joining $q^{j}$ and $z^{j}$. As we travel along $\gamma^{j}$, there is a first point $\alpha^{j}$ on the curve with $\alpha^{j} \in \partial V \cap D^{j}$. Let $\sigma^{j}$ be the subcurve of $\gamma^{j}$ with end-points $q^{j}$ and $\alpha^{j}$. Then $\sigma^{j}$ is contained in an $\epsilon$ - neighbourhood of $\partial D^{j}$ for $\epsilon>0$ small and for all $j$ large. Using (5.12) we get after integration,

$$
\int_{0}^{1} F_{D^{j}}^{K}\left(\gamma^{j}(t), \dot{\gamma}^{j}(t)\right) d t \geq \int_{0}^{1} F_{D^{j}}^{K}\left(\sigma^{j}(t), \dot{\sigma}^{j}(t)\right) d t \geq C \int_{0}^{1} \frac{\left|\dot{\sigma}^{j}(t)\right|}{d\left(\sigma^{j}(t), \partial D^{j}\right)^{1 / 2 m}} \gtrsim \frac{1}{\epsilon^{1 / 2 m}}
$$

Taking infimum over all admissible curve $\gamma^{j}$ yields

$$
d_{D^{j}}\left(q^{j}, z^{j}\right) \gtrsim \epsilon^{-1 / 2 m} \text {. }
$$

which violates the fact that $q^{j} \in B_{D^{j}}\left(z^{j}, R\right)$ for $\epsilon$ small enough. This completes the proof of the lemma. 


\section{Lemma 5.7.}

$$
\lim _{j \rightarrow \infty} d_{D^{j}}\left(z^{j}, \cdot\right)=d_{D_{\infty}}\left(z^{0}, \cdot\right)
$$

Moreover, the convergence is uniform on compact sets of $D_{\infty}$.

Proof. Let $K$ be a compact subdomain of $D_{\infty}$ and suppose that the desired convergence does not occur. Then there exists a $\epsilon_{0}>0$ and a sequence of points $\left\{q^{j}\right\} \subset K$ which is relatively compact in $D^{j}$ for all $j$ large such that

$$
\left|d_{D^{j}}\left(z^{j}, q^{j}\right)-d_{D_{\infty}}\left(z^{0}, q^{j}\right)\right|>\epsilon_{0} .
$$

By passing to a subsequence, we may assume that $q^{j} \rightarrow q^{0} \in K$ as $j \rightarrow \infty$. Then using the continuity of $d_{D_{\infty}}\left(z^{0}, \cdot\right)$ we have

$$
\left|d_{D^{j}}\left(z^{j}, q^{j}\right)-d_{D_{\infty}}\left(z^{0}, q^{0}\right)\right|>\epsilon_{0} / 2
$$

for all $j$ large. Fix $\epsilon>0$ arbitrarily small. It is easy to see that

$$
d_{D^{j}}\left(z^{0}, q^{0}\right) \leq d_{D_{\infty}}\left(z^{0}, q^{0}\right)+\epsilon / 2
$$

for all $j$ large. Let $B\left(z^{0}, \delta_{1}\right)$ and $B\left(q^{0}, \delta_{2}\right)$ be sufficiently small neighbourhoods of $z^{0}$ and $q^{0}$ respectively which are compactly contained in $D^{j}$ for all large $j$. It now follows that

$$
\begin{aligned}
d_{D^{j}}\left(z^{j}, q^{j}\right) & \leq d_{D^{j}}\left(z^{j}, z^{0}\right)+d_{D^{j}}\left(z^{0}, q^{0}\right)+d_{D^{j}}\left(q^{0}, q^{j}\right) \\
& \leq d_{B\left(z^{0}, \delta_{1}\right)}\left(z^{j}, z^{0}\right)+d_{D^{j}}\left(z^{0}, q^{0}\right)+d_{B\left(q^{0}, \delta_{2}\right)}\left(q^{0}, q^{j}\right) \\
& \leq d_{D_{\infty}}\left(z^{0}, q^{0}\right)+\epsilon .
\end{aligned}
$$

for all $j$ large. The second inequality uses the distance decreasing property of the Kobayashi metric. The third inequality follows from (5.13) and the following observation: since $z^{j} \rightarrow z^{0}$ and the domains $D^{j}$ converge to $D_{\infty}$, it follows that the ball $B\left(z^{0}, \delta_{1}\right)$ contains $z^{j}$ for large $j$ and is contained in $D^{j}$ for all large $j$. Thus

$$
d_{D^{j}}\left(z^{j}, z^{0}\right) \leq d_{B\left(z^{0}, \delta_{1}\right)}\left(z^{j}, z^{0}\right) \lesssim\left|z^{j}-z^{0}\right| .
$$

The same argument works for showing that $d_{D^{j}}\left(q^{0}, q^{j}\right)$ is small. Hence

$$
d_{D^{j}}\left(z^{j}, q^{j}\right) \leq d_{D_{\infty}}\left(z^{0}, q^{0}\right)+\epsilon
$$

for all $j$ large. For the converse, we intend to use lemma 5.4. First recall from (5.5) that for each $\epsilon>0$

$$
B_{D_{\infty}}\left(z^{0}, R-\epsilon\right) \subset B_{D^{j}}\left(z^{j}, R\right)
$$

for all $R>0$ and for all $j$ large. The Kobayashi completeness of $D_{\infty}$ implies that

$$
D_{\infty}=\bigcup_{\nu=1}^{\infty} B_{D_{\infty}}\left(z^{0}, \nu\right)
$$

i.e., $D_{\infty}$ can be exhausted by an increasing union of relatively compact domains $B_{D_{\infty}}\left(z^{0}, \nu\right)$. As a result, there exist uniform positive constants $\nu^{0}$ and $\tilde{R}$ depending only on $K$ such that

$$
K \subset B_{D_{\infty}}\left(z^{0}, \nu^{0}\right) \subset B_{D^{j}}\left(z^{j}, \tilde{R}\right)
$$

for all $j$ large. By lemma 5.6

$$
d_{D_{\infty}}\left(z^{j}, q^{j}\right) \leq d_{B_{D^{j}}\left(z^{j}, R^{\prime}\right)}\left(z^{j}, q^{j}\right)
$$

where $R^{\prime}>0$ is chosen such that $R^{\prime} \gg 2 \tilde{R}$. Now, apply lemma 5.4 to the domain $D^{j}$. Let the Kobayashi metric ball $B_{D^{j}}\left(z^{j}, R^{\prime}\right)$ play the role of the subdomain $D^{\prime}$. Then

$$
d_{B_{D^{j}}\left(z^{j}, R^{\prime}\right)}\left(z^{j}, q^{j}\right) \leq \frac{d_{D^{j}}\left(z^{j}, q^{j}\right)}{\tanh \left(R^{\prime} / 2-d_{D^{j}}\left(z^{j}, q^{j}\right)\right)} .
$$

Since $q^{j} \in B_{D^{j}}\left(z^{j}, \tilde{R}\right)$ for all $j$ large and tanh is increasing on $[0, \infty)$, it follows that

$$
d_{D_{\infty}}\left(z^{j}, q^{j}\right) \leq \frac{d_{D^{j}}\left(z^{j}, q^{j}\right)}{\tanh \left(R^{\prime} / 2-\tilde{R}\right)}
$$

Letting $R^{\prime} \rightarrow \infty$ yields

$$
d_{D_{\infty}}\left(z^{j}, q^{j}\right) \leq \frac{d_{D^{j}}\left(z^{j}, q^{j}\right)}{1-\epsilon}
$$


for all $j$ large. Again exploiting the continuity of $d_{D_{\infty}}(\cdot, \cdot)$ and (5.14), we see that

$$
d_{D_{\infty}}\left(z^{0}, q^{0}\right) \leq d_{D^{j}}\left(z^{j}, q^{j}\right)+C \epsilon
$$

for all $j$ large. Combining the estimates (5.15) and (5.16), we get

$$
\lim _{j \rightarrow \infty} d_{D^{j}}\left(z^{j}, q^{j}\right)=d_{D_{\infty}}\left(z^{0}, q^{0}\right) .
$$

This is a contradiction and hence the result follows.

The following is an immediate corollary of the above result.

\section{Corollary 5.8.}

$$
\lim _{j \rightarrow \infty} d_{D^{j}}\left(z^{0}, \cdot\right)=d_{D_{\infty}}\left(z^{0}, \cdot\right)
$$

Moreover, the convergence is uniform on compact sets of $D_{\infty}$.

Proof. For all $w$ in a fixed compact set $K$ of $D_{\infty}$, we have

$$
\left|d_{D^{j}}\left(z^{0}, w\right)-d_{D_{\infty}}\left(z^{0}, w\right)\right| \leq\left|d_{D^{j}}\left(z^{0}, w\right)-d_{D^{j}}\left(z^{j}, w\right)\right|+\left|d_{D^{j}}\left(z^{j}, w\right)-d_{D_{\infty}}\left(z^{0}, w\right)\right|
$$

It follows from lemma 5.7 that

$$
\left|d_{D^{j}}\left(z^{j}, w\right)-d_{D_{\infty}}\left(z^{0}, w\right)\right| \rightarrow 0
$$

uniformly for all $w \in K$ as $j \rightarrow \infty$. Now, let $B\left(z^{0}, \delta\right)$ be a sufficiently small neighbourhood of $z^{0}$. Then the triangle inequality gives us that

$$
\left|d_{D^{j}}\left(z^{0}, w\right)-d_{D^{j}}\left(z^{j}, w\right)\right| \leq d_{D^{j}}\left(z^{0}, z^{j}\right) \leq d_{B\left(z^{0}, \delta\right)}\left(z^{0}, z^{j}\right) \rightarrow 0
$$

as $j \rightarrow \infty$. This finishes the proof.

We record the following consequence of corollary 5.8. The proof is exactly as that of lemma 4.4 and is hence omitted.

Lemma 5.9. For all $R>0$, the sequence of domains $\left\{B_{D^{j}}\left(z^{0}, R\right)\right\}$ converges in the Hausdorff metric to $B_{D_{\infty}}\left(z^{0}, R\right)$. Moreover, for any $\epsilon>0$ and for all $j$ large

- $B_{D_{\infty}}\left(z^{0}, R\right) \subset B_{D^{j}}\left(z^{0}, R+\epsilon\right)$,

- $B_{D^{j}}\left(z^{0}, R-\epsilon\right) \subset B_{D_{\infty}}\left(z^{0}, R\right)$.

Proof of Theorem 5.1(ii): Since $h$ is invariant under biholomorphisms,

$$
h_{D}\left(p^{j}\right)=h_{D^{j}}\left(z^{0}\right)
$$

for all $j$. Hence, it suffices to show that

$$
\lim _{j \rightarrow \infty} h_{D^{j}}\left(z^{0}\right)=h_{D_{\infty}}\left(z^{0}\right)
$$

Fix $\epsilon>0$ arbitrarily small and let $R>0$ be such that

$$
h_{D_{\infty}}\left(z^{0}\right)>\frac{1}{R}-\epsilon .
$$

There exists a biholomorphic imbedding $F: \mathbf{B}^{2} \rightarrow D_{\infty}$ such that $F(0)=z^{0}$ and $B_{D_{\infty}}\left(z^{0}, R\right) \subset F\left(\mathbf{B}^{2}\right)$. There exists a $\delta>0$ such that $F\left((1-\delta) \mathbf{B}^{2}\right) \supset B_{D_{\infty}}\left(z^{0}, R-\epsilon\right)$. Since $F\left((1-\delta) \mathbf{B}^{2}\right)$ is compactly contained in $D_{\infty}, F\left((1-\delta) \mathbf{B}^{2}\right) \subset D^{j}$ for all $j$ large. It follows from lemma 5.9 that

$$
B_{D^{j}}\left(z^{0}, R-2 \epsilon\right) \subset B_{D_{\infty}}\left(z^{0}, R-\epsilon\right)
$$

for all $j$ large. As a consequence,

$$
B_{D^{j}}\left(z^{0}, R-2 \epsilon\right) \subset F\left((1-\delta) \mathbf{B}^{2}\right) \subset D^{j}
$$

or equivalently

for all $j$ large. This implies that

$$
h_{D^{j}}\left(z^{0}\right) \leq \frac{1}{R-2 \epsilon}
$$

$$
\limsup _{j \rightarrow \infty} h_{D^{j}}\left(z^{0}\right) \leq h_{D_{\infty}}\left(z^{0}\right)
$$


Conversely, there exist a sequence of biholomorphic imbeddings $F^{j}: \mathbf{B}^{2} \rightarrow D^{j}$ and positive numbers $R_{j}$ such that $F^{j}(0)=z^{0}, B_{D^{j}}\left(z^{0}, R_{j}\right) \subset F^{j}\left(\mathbf{B}^{2}\right)$ and

$$
h_{D^{j}}\left(z^{0}\right) \geq \frac{1}{R_{j}}-\epsilon .
$$

The sequence $\left\{F^{j}\right\}$ admits a subsequence that converges uniformly on compact sets of $\mathbf{B}^{2}$ to a holomorphic mapping $F: \mathbf{B}^{2} \rightarrow D_{\infty}$. Indeed, consider the mappings

$$
\left(\Delta_{\zeta^{j}}^{\epsilon_{j}} \circ \phi^{\zeta^{j}}\right)^{-1} \circ F^{j}: \mathbf{B}^{2} \rightarrow D
$$

Observe that

$$
\left(\Delta_{\zeta^{j}}^{\epsilon_{j}} \circ \phi^{\zeta^{j}}\right)^{-1} \circ F^{j}(0)=p^{j} \rightarrow p^{0} \quad \text { as } j \rightarrow \infty
$$

and

$$
\left(\Delta_{\zeta^{j}}^{\epsilon_{j}} \circ \phi^{\zeta^{j}}\right)^{-1} \circ F^{j}(0) \in Q\left(\zeta^{j}, C_{1} \epsilon_{j}\right)
$$

for some constant $C_{1} \geq 1$. For $r \in(0,1)$ fixed, it follows from 4 that there exists a positive constant $C_{2}=C_{2}(r)$ such that

$$
\left(\Delta_{\zeta^{j}}^{\epsilon_{j}} \circ \phi^{\zeta^{j}}\right)^{-1} \circ F^{j}\left(\mathbf{B}^{2}(0, r)\right) \subset Q\left(\zeta^{j}, C_{1} C_{2} \epsilon_{j}\right)
$$

which exactly means that

$$
F^{j}\left(\mathbf{B}^{2}(0, r)\right) \subset \Delta\left(0, \frac{\tau\left(\zeta^{j}, C_{1} C_{2} \epsilon_{j}\right)}{\tau\left(\zeta^{j}, \epsilon_{j}\right)}\right) \times \Delta\left(0, \frac{C_{1} C_{2} \epsilon_{j}}{\epsilon_{j}}\right) \subset \Delta\left(0, \sqrt{C_{1} C_{2}}\right) \times \Delta\left(0, C_{1} C_{2}\right) .
$$

This shows that the sequence $\left\{F^{j}\right\}$ is a normal family. Hence, $\left\{F^{j}\right\}$ admits a subsequence that converges uniformly on compact sets of $\mathbf{B}^{2}$ to a holomorphic mapping $F: \mathbf{B}^{2} \rightarrow \mathbf{C}^{2}$ or $F \equiv \infty$. The latter is not possible since $F(0)=\lim _{j \rightarrow \infty} F^{j}(0)=z^{0}$. As before, we can infer that $F: \mathbf{B}^{2} \rightarrow D_{\infty}$. Note that

$$
\frac{1}{R_{j}} \leq h_{D^{j}}\left(z^{0}\right)+\epsilon \leq h_{D_{\infty}}\left(z^{0}\right)<\infty
$$

for all $j$ large. Hence, we may assume that the sequence $\left\{R_{j}\right\}$ converges to some $R_{0}>0$. It follows that

$$
B_{D^{j}}\left(z^{0}, R_{0}-\epsilon\right) \subset B_{D^{j}}\left(z^{0}, R_{j}\right) \subset F^{j}\left(\mathbf{B}^{2}\right)
$$

for all $j$ large. Also, lemma 5.9 implies that

$$
B_{D_{\infty}}\left(z^{0}, R_{0}-2 \epsilon\right) \subset B_{D^{j}}\left(z^{0}, R_{0}-\epsilon\right)
$$

for all $j$ large. Combining (5.19) and (5.20), we get

$$
B_{D_{\infty}}\left(z^{0}, R_{0}-2 \epsilon\right) \subset F\left(\mathbf{B}^{2}\right) .
$$

Therefore, $F$ is non-constant. It follows from Hurwitz's theorem that $F$ is injective on $\mathbf{B}^{2}$ and hence

$$
h_{D_{\infty}}\left(z^{0}\right) \leq 1 /\left(R_{0}-2 \epsilon\right)
$$

which together with (5.18) implies that

$$
h_{D_{\infty}}\left(z^{0}\right) \leq \liminf _{j \rightarrow \infty} h_{D^{j}}\left(z^{0}\right)
$$

It follows from (5.17) and (5.21) that

$$
h_{D^{j}}\left(z^{0}\right) \rightarrow h_{D_{\infty}}\left(z^{0}\right)
$$

as $j \rightarrow \infty$. This completes the proof of theorem 5.1 


\section{BeHAVIOUR OF $h$ NEAR CONVEX FINITE TYPE BOUNDARY POINTS}

The main result is as follows:

Theorem 6.1. Let $D \subset \mathbf{C}^{n}$ be a smoothly bounded convex domain of finite type. Let $\left\{q^{j}\right\}$ be a sequence of points in $D$ converging to $q^{0} \in \partial D$. Then

$$
h_{D}\left(q^{j}\right) \rightarrow h_{D_{\infty}}\left(\left({ }^{\prime} 0,-1\right)\right)
$$

as $j \rightarrow \infty$ where $D_{\infty}$ is a biholomorph of the limiting domain $D_{0}$ obtained by scaling $D$ with respect to the sequence $\left\{q^{j}\right\}$.

As in section 5, there are two cases to be considered, i.e., after passing to a subsequence if needed,

(i) $\lim _{j \rightarrow \infty} h_{D}\left(p^{j}\right)=0$, or

(ii) $\lim _{j \rightarrow \infty} h_{D}\left(p^{j}\right)>c$ for some positive constant $c$.

Here too the domain $D_{\infty}$ will be biholomorphic to $\mathbf{B}^{n}$ in case (i) while this will not be the case in (ii).

In order to be able to prove the above result, we need to introduce the following special coordinates constructed for convex finite type domains in [27] (see 28] also).

Let $D \subset \mathbf{C}^{n}$ be as stated above. Assume that $q^{0}=0$ without loss of generality. Let $D=\{\rho(z, \bar{z})<0\}$ where $\rho$ is a smooth defining function for $\partial D$ which has the form

$$
\rho(z, \bar{z})=\Re z_{n}+\psi\left({ }^{\prime} z, \Im z_{n}\right)
$$

near the origin with $\psi$ a smooth convex function (i.e., the real Hessian of $\psi$ is positive semi-definite). We may assume that $\rho$ has the property that all the sets $\{z: \rho(z)<\eta\}$ are convex for some $\eta$ in some range $-\eta_{0}<\eta<\eta_{0}, \eta_{0}>0$. For $q \in D$ sufficiently close to $\partial D$, let

$$
D_{q, \epsilon}=\{z: \rho(z)<\rho(q)+\epsilon\} .
$$

Working with sufficiently small $\epsilon>0$, there is a unique point $p_{q, \epsilon}^{n} \in \partial D_{q, \epsilon}$ where the distance of $q$ to $\partial D_{q, \epsilon}$ is achieved. Denote the complex line containing $q$ and $p_{q, \epsilon}^{n}$ by $L_{n}$ and let $\tau_{n}(q, \epsilon)=\left|q-p_{q, \epsilon}^{n}\right|$. Consider $\left(L_{n}\right)^{\perp}$ the orthogonal complement of the complex line $L_{n}$ in $\mathbf{C}^{n}$. Since $\partial D$ is of finite type, the distance from $q$ to $\partial D_{q, \epsilon}$ along each complex line in $\left(L_{n}\right)^{\perp}$ is uniformly bounded. Let $\tau_{n-1}(q, \epsilon)$ be the largest such distance and $p_{q, \epsilon}^{n-1} \in \partial D_{q, \epsilon}$ be any point such that $\left|q-p_{q, \epsilon}^{n-1}\right|=\tau_{n-1}(q, \epsilon)$. Denote the complex line containing $q$ and $p_{q, \epsilon}^{n-1}$ by $L_{n-1}$. Now consider the orthogonal complement of the C-subspace spanned by $L_{n}$ and $L_{n-1}$ and find the largest distance from $q$ to $\partial D_{q, \epsilon}$ therein. There exists $p_{q, \epsilon}^{n-2} \in \partial D_{q, \epsilon}$ where this distance is achieved. Let $\tau_{n-2}(q, \epsilon)=\left|q-p_{q, \epsilon}^{n-2}\right|$ and $L_{n-2}$ denote the complex line containing $q$ and $p_{q, \epsilon}^{n-2}$. Repeating this process, we get orthogonal lines $L_{n}, L_{n-1}, \ldots, L_{1}$. Let $T^{q, \epsilon}$ be the translation sending $q$ to the origin and $U^{q, \epsilon}$ be a unitary mapping of $\mathbf{C}^{n}$ sending $L_{i}$ to the $z_{i}$-axis and $p_{q, \epsilon}^{i}-q$ to a point on the $\Re z_{i}$-axis. Note that

$$
\begin{aligned}
U^{q, \epsilon} \circ T^{q, \epsilon}(q) & =0 \\
\text { and } \quad U^{q, \epsilon} \circ T^{q, \epsilon}\left(p_{q, \epsilon}^{i}\right) & =\left(0, \ldots, \tau_{i}(q, \epsilon), \ldots, 0\right)
\end{aligned}
$$

for all $1 \leq i \leq n$. Denote the new coordinates by

$$
\left(z_{1}^{q, \epsilon}, \ldots, z_{n}^{q, \epsilon}\right)=U^{q, \epsilon} \circ T^{q, \epsilon}\left(z_{1}, \ldots, z_{n}\right)
$$

and the corresponding defining function is given by

$$
\rho^{q, \epsilon}=\rho \circ\left(U^{q, \epsilon} \circ T^{q, \epsilon}\right)^{-1} .
$$

Moreover, McNeal defined the polydiscs $P(q, \epsilon)$ in the new coordinates $\left(z_{1}^{q, \epsilon}, \ldots, z_{n}^{q, \epsilon}\right)$ centered at $q$ as

$$
P(q, \epsilon)=\left\{\left(z_{1}^{q, \epsilon}, \ldots, z_{n}^{q, \epsilon}\right):\left|z_{1}^{q, \epsilon}\right|<\tau_{1}(q, \epsilon), \ldots,\left|z_{n}^{q, \epsilon}\right|<\tau_{n}(q, \epsilon)\right\} .
$$

Although we may not write explicitly all the time, the reader must be aware of the dependence of all the coordinates, points and numbers on $q$ and $\epsilon$. Having recalled certain basic facts about the local geometry of convex domains of finite type, we briefly describe the scaling of the domain.

\section{Scaling the domain $D$ :}


Let $\left\{q^{j}\right\}$ be a sequence of points in $D$ accumulating at $q^{0}=\left({ }^{\prime} 0,0\right) \in \partial D$. Set $\epsilon_{j}=-\rho\left(q^{j}\right)$. The positive numbers $\tau_{1}\left(q^{j}, \epsilon_{j}\right), \ldots, \tau_{n}\left(q^{j}, \epsilon_{j}\right)$ and $p^{1, j}, \ldots, p^{n, j}$ are those associated with $q^{j}$ and $\epsilon_{j}$. Define the dilations

$$
\Lambda_{q^{j}}^{\epsilon_{j}}(z)=\left(\tau_{1}\left(q^{j}, \epsilon_{j}\right) z_{1}, \ldots, \tau_{n}\left(q^{j}, \epsilon_{j}\right) z_{n}\right)
$$

and the dilated domains $D^{j}=\left(\Lambda_{q^{j}}^{\epsilon_{j}}\right)^{-1} \circ U^{q^{j}, \epsilon_{j}} \circ T^{q^{j}, \epsilon_{j}}(D)$ are defined by

$$
\rho^{j}(z)=\rho \circ\left(U^{q^{j}, \epsilon_{j}} \circ T^{q^{j}, \epsilon_{j}}\right)^{-1} \circ \Lambda_{q^{j}}^{\epsilon_{j}}(z)
$$

Note that $D^{j}$ is convex and $\left({ }^{\prime} 0,0\right) \in D^{j}$ for all $j$. Among other things, the following two claims were proved in [15. First, that $D^{j}$ converges to

$$
D_{0}=\left\{\left({ }^{\prime} z, z_{n}\right) \in \mathbf{C}^{n}: \tilde{\rho}(z)=-1+\Re\left(\sum_{k=1}^{n} b_{k} z_{k}\right)+P\left({ }^{\prime} z\right)<0\right\}
$$

where $b_{k}$ are complex numbers and $P$ is a real convex polynomial of degree less than or equal to $2 m$. Secondly, for all large $j, D^{j}$ and hence $D_{0}$ are contained in the intersection of half spaces $H_{1} \cap H_{2} \cap \ldots \cap H_{n}$, where

and for $k<n$

$$
H_{n}=\left\{z \in \mathbf{C}^{n}: \Re\left(\left(z_{n}-1\right) \frac{\partial \tilde{\rho}}{\partial z_{n}}\left(e^{n}\right)\right) \leq 0\right\}
$$

$$
H_{k}=\left\{z \in \mathbf{C}^{n}: \Re\left(\left(z_{k}-1\right) \frac{\partial \tilde{\rho}}{\partial z_{k}}\left(e^{k}\right)+\sum_{i=k+1}^{n} \frac{\partial \tilde{\rho}}{\partial z_{i}}\left(e^{k}\right) z_{i}\right) \leq 0\right\}
$$

where

$$
e^{i}=\left(\Lambda_{q^{j}}^{\epsilon_{j}}\right)^{-1}\left(p^{i, j}\right) \quad \text { and } \quad \frac{\partial \tilde{\rho}}{\partial z_{i}}\left(e^{i}\right) \in \mathbf{R} \backslash\{0\} \text { for all } 1 \leq i \leq n .
$$

As a consequence, there exists a rational biholomorphism from $D_{0}$ to a bounded domain contained in the polydisc (the Cayley transformation in each variable). In particular, $D_{0}$ is hyperbolic. Therefore, $D_{0}$ is Brody hyperbolic, i.e., $D_{0}$ contains no nontrivial complex affine line. Then there is no complex line in $\partial D_{0}$ and according to theorem 1.1 of [27, $D_{0}$ is of finite type and $P$ is nondegenerate. It follows that $D_{0}$ is complete hyperbolic.

Moreover, it follows from [27] that the constant $b_{n}$ is different from zero. Then by a $\mathbf{C}$-affine change of coordinates, $D_{0}$ is equivalent to the convex domain $D_{\infty}=\left\{\left({ }^{\prime} w, w_{n}\right) \in \mathbf{C}^{n}: 2 \Re w_{n}+P\left({ }^{\prime} w\right)<0\right\}$. As a result,

$$
h_{D_{\infty}}\left(\left({ }^{\prime} 0,-1\right)\right)=h_{D_{0}}\left(\left({ }^{\prime} 0,0\right)\right) .
$$

In the particular case when the sequence $\left\{q^{j}\right\}$ converges normally to the point $q^{0}$. We denote the multitype of $\partial D$ at the origin by $\mathcal{M}(\partial D, 0)=\left(m_{1}, \ldots, m_{n-1}, 1\right)$; the points $q^{j}$ will be $\left({ }^{\prime} 0,-\epsilon_{n}\right)$ in the coordinates defined by $\mathrm{Yu}$ in [40]. Thus we may assume that for all $j, \tau\left(q^{j}, \epsilon_{j}\right)=\epsilon_{j}$ and the function $\rho$ is defined in a fixed neighbourhood of the origin by

$$
\rho\left({ }^{\prime} z, z_{n}\right)=2 \Re z_{n}+P_{0}\left({ }^{\prime} z\right)+R(z)
$$

where $P_{0}$ is a nondegenerate weighted homogeneous polynomial of degree 1 with respect to the weights $\mathcal{M}(\partial D, 0)$ and $R$ denotes terms of degree at least two. In this case, it turns out that $P\left({ }^{\prime} z\right)=P_{0}\left({ }^{\prime} z\right)$ so that the limiting domain $D_{\infty}$ is a biholomorph of the domain

$$
\left\{\left({ }^{\prime} z, z_{n}\right) \in \mathbf{C}^{n}: 2 \Re z_{n}+P_{0}\left({ }^{\prime} z\right)<0\right\} .
$$

Now, arguments similar to those in lemma 5.2 using the fact that the domains $D^{j}$ are all contained in $H_{1} \cap H_{2} \cap \ldots \cap H_{n}$ for large $j$, shows that

Lemma 6.2. For $(a, v) \in D_{0} \times \mathbf{C}^{n}$,

$$
\lim _{j \rightarrow \infty} F_{D^{j}}^{K}(a, v)=F_{D_{0}}^{K}(a, v) .
$$

Moreover, the convergence is uniform on compact sets of $D_{0} \times \mathbf{C}^{n}$. 


\section{Stability of the Kobayashi distance:}

The goal now will be to recover the behaviour of the distance function related to the Kobayashi metric. To do this, we use ideas from [36. A key ingredient will be Lempert's theorem (see [26]) on the existence of complex geodesics in strictly convex domains. Write $\left({ }^{\prime} 0,0\right)=z^{0}$ for brevity.

\section{Lemma 6.3.}

$$
\lim _{j \rightarrow \infty} d_{D^{j}}\left(z^{0}, \cdot\right)=d_{D_{0}}\left(z^{0}, \cdot\right) .
$$

Moreover, the convergence is uniform on compact sets of $D_{0}$.

Proof. Let $K \subset D_{0}$ be compact and suppose that the desired convergence does not occur. Then there exists a $\epsilon_{0}>0$ and a sequence of points $\left\{p^{j}\right\} \subset K$ which is relatively compact in $D^{j}$ for $j$ large such that

$$
\left|d_{D^{j}}\left(z^{0}, p^{j}\right)-d_{D_{0}}\left(z^{0}, p^{j}\right)\right|>\epsilon_{0}
$$

for all $j$ large. By passing to a subsequence, we may assume that $p^{j} \rightarrow p^{0} \in K$ as $j \rightarrow \infty$. Since $d_{D_{0}}\left(z^{0}, \cdot\right)$ is continuous, it follows that

$$
\left|d_{D^{j}}\left(z^{0}, p^{j}\right)-d_{D_{0}}\left(z^{0}, p^{0}\right)\right|>\epsilon_{0} / 2
$$

for all $j$ large. Fix $\epsilon>0$ and let $\gamma:[0,1] \rightarrow D_{0}$ be a path such that $\gamma(0)=z^{0}, \gamma(1)=p^{0}$ and

$$
\int_{0}^{1} F_{D_{0}}^{K}(\gamma(t), \dot{\gamma}(t)) d t<d_{D_{0}}\left(z^{0}, p^{0}\right)+\epsilon / 2 \text {. }
$$

Define $\gamma^{j}:[0,1] \rightarrow \mathbf{C}^{n}$ by

$$
\gamma^{j}(t)=\gamma(t)+\left(p^{j}-p^{0}\right) t
$$

Since the image $\gamma([0,1])$ is compactly contained in $D_{0}$ and $p^{j} \rightarrow p^{0} \in K$ as $j \rightarrow \infty$, it follows that $\gamma^{j}:[0,1] \rightarrow D^{j}$ for $j$ large. In addition, $\gamma^{j}(0)=\gamma(0)=z^{0}$ and $\gamma^{j}(1)=\gamma(1)+p^{j}-p^{0}=p^{j}$.

Note that $\gamma^{j} \rightarrow \gamma$ and $\dot{\gamma}^{j} \rightarrow \dot{\gamma}$ uniformly on $[0,1]$. Further, it is already known from lemma 6.2 that $F_{D^{j}}^{K}(\cdot, \cdot) \rightarrow F_{D_{0}}^{K}(\cdot, \cdot)$ uniformly on compact sets of $D_{0} \times \mathbf{C}^{n}$ Therefore for $j$ large, we obtain

$$
\int_{0}^{1} F_{D^{j}}^{K}\left(\gamma^{j}(t), \dot{\gamma}^{j}(t)\right) d t \leq \int_{0}^{1} F_{D_{0}}^{K}(\gamma(t), \dot{\gamma}(t)) d t+\epsilon / 2<d_{D_{0}}\left(z^{0}, p^{0}\right)+\epsilon .
$$

By definition of $d_{D^{j}}\left(z^{0}, p^{j}\right)$ it follows that

$$
d_{D^{j}}\left(z^{0}, p^{j}\right) \leq \int_{0}^{1} F_{D^{j}}^{K}\left(\gamma^{j}(t), \dot{\gamma}^{j}(t)\right) d t \leq d_{D_{0}}\left(z^{0}, p^{0}\right)+\epsilon .
$$

Thus

$$
\limsup _{j \rightarrow \infty} d_{D^{j}}\left(z^{0}, p^{j}\right) \leq d_{D_{0}}\left(z^{0}, p^{0}\right) .
$$

Conversely, it follows by Lempert's work that there exist $m_{j}>1$ and holomorphic mappings

$$
\phi^{j}: \Delta\left(0, m_{j}\right) \rightarrow D^{j}
$$

such that $\phi^{j}(0)=z^{0}, \phi^{j}(1)=p^{j}$ and

Therefore,

$$
d_{\Delta\left(0, m_{j}\right)}(0,1)=d_{D^{j}}\left(z^{0}, p^{j}\right)=\int_{0}^{1} F_{D^{j}}^{K}\left(\phi^{j}(t), \dot{\phi}^{j}(t)\right) d t .
$$

$$
\frac{1}{2} \log \left(\frac{m_{j}+1}{m_{j}-1}\right)=d_{\Delta\left(0, m_{j}\right)}(0,1)=d_{D^{j}}\left(z^{0}, p^{j}\right)
$$

However from (6.3) we have that

$$
d_{D^{j}}\left(z^{0}, p^{j}\right) \leq d_{D_{0}}\left(z^{0}, p^{0}\right)+\epsilon<\infty
$$

and hence $m_{j}>1+\delta$ for some uniform $\delta>0$ for all $j$ large. Moreover, the domains $D^{j}$ are all contained in the intersection of the half planes. Hence the family $\left.\phi^{j}\right|_{\Delta(0,1+\delta)}: \Delta(0,1+\delta) \rightarrow D^{j}$ is normal. Consequently, the limit map $\phi: \Delta(0,1+\delta) \rightarrow \bar{D}_{0}$. Note that by construction $\phi(0)=z^{0}$ and $\phi(1)=p^{0}$. Using the maximum principle, we conclude that $\phi: \Delta(0,1+\delta) \rightarrow D_{0}$. 
Since $\phi^{j} \rightarrow \phi$ and $\dot{\phi}^{j} \rightarrow \dot{\phi}$ uniformly on $[0,1]$, again exploiting the uniform convergence of $F_{D^{j}}^{K}(\cdot, \cdot) \rightarrow$ $F_{D_{0}}^{K}(\cdot, \cdot)$ on compact sets of $D_{0} \times \mathbf{C}^{n}$, we see that

$$
\int_{0}^{1} F_{D_{0}}^{K}(\phi(t), \dot{\phi}(t)) d t \leq \int_{0}^{1} F_{D^{j}}^{K}\left(\phi^{j}(t), \dot{\phi}^{j}(t)\right) d t+\epsilon=d_{D^{j}}\left(z^{0}, p^{j}\right)+\epsilon
$$

for all $j$ large. Finally, observe that $\left.\phi\right|_{[0,1]}$ is a differentiable path in $D_{0}$ joining $z^{0}$ and $p^{0}$. Hence by definition

$$
d_{D_{0}}\left(z^{0}, p^{0}\right) \leq \int_{0}^{1} F_{D_{0}}^{K}(\phi(t), \dot{\phi}(t)) d t \leq d_{D^{j}}\left(z^{0}, p^{j}\right)+\epsilon
$$

Combining (6.3) and (6.4) shows that

$$
\lim _{j \rightarrow \infty} d_{D^{j}}\left(z^{0}, p^{j}\right)=d_{D_{0}}\left(z^{0}, p^{0}\right)
$$

which contradicts the assumption (6.2) and proves the required result.

Lemma 6.4. For all $R>0$, the sequence of domains $\left\{B_{D^{j}}\left(z^{0}, R\right)\right\}$ converges in the Hausdorff metric to $B_{D_{0}}\left(z^{0}, R\right)$. Moreover, for any $\epsilon>0$ and for all $j$ large

- $B_{D_{0}}\left(z^{0}, R\right) \subset B_{D^{j}}\left(z^{0}, R+\epsilon\right)$,

- $B_{D^{j}}\left(z^{0}, R-\epsilon\right) \subset B_{D_{0}}\left(z^{0}, R\right)$.

Proof. This follows by making the relevant changes in the proof of lemma 4.4.

Proof of theorem 6.1: Using lemma 6.3 and 6.4 an argument similar to the one employed in theorem 5.1 (ii) can be used to complete the proof of theorem 6.1

\section{BehaViour of $h$ NEAR THE CORNERs of A GENERIC ANALYTIC POLYHEDRON}

To investigate the behaviour of $h_{P}\left(\cdot, \Delta^{n}\right)$ near a singular boundary point of an analytic poyhedron, we use the rescaling technique given in [24].

Theorem 7.1. Let $D \subset \mathbf{C}^{n}$ be a bounded domain. Let $z^{0} \in \partial D$ have a neighbourhood $U$ such that $U \cap \partial D=\left\{z \in \mathbf{C}^{n}:\left|f^{i}(z)\right|<1\right.$, for all $\left.1 \leq i \leq n\right\}$ and $\left|f^{i}\left(z^{0}\right)\right|=1$ for all $1 \leq i \leq n$ where $f^{i} \in \mathcal{O}(U)$ for all $1 \leq i \leq n$. Assume that $d f^{1} \wedge \ldots \wedge d f^{n} \neq 0$ at $z^{0}$. Then $h_{D}\left(z, \Delta^{n}\right) \rightarrow 0$ as $z \rightarrow z^{0}$.

Proof. Let $\left\{z^{k}\right\} \subset D$ be a sequence converging to $z^{0}$. It suffices to show that for any sequence $h_{D}\left(z^{k}\right) \rightarrow 0$ as $k \rightarrow \infty$. An application of the implicit function theorem gives an open neighbourhood $U$ of $z^{0}$ in $\mathbf{C}^{n}$ and real numbers $\theta_{i}, i=1, \ldots, n$ satisfying the following properties:

- $F:=\left(e^{\iota \theta_{1}} f^{1}, \ldots, e^{\iota \theta_{n}} f^{n}\right): U \rightarrow \mathbf{C}^{n}$ is a biholomorphic imbedding,

- $F\left(z^{0}\right)=(1, \ldots, 1) \in \mathbf{C}^{n}$,

- $F(U \cap D)=\Delta^{n} \cap F(U)$.

Now, consider $\phi: \Delta^{n} \rightarrow \mathbf{H}^{n}=\mathbf{H} \times \ldots \times \mathbf{H}$ defined by

$$
\begin{aligned}
\phi(z) & =\left(\phi_{1}(z), \ldots, \phi_{n}(z)\right) \\
& =\left(\iota \frac{1-z_{1}}{1+z_{1}}, \ldots, \iota \frac{1-z_{n}}{1+z_{n}}\right)
\end{aligned}
$$

where $\mathbf{H}=\{w \in \mathbf{C}: \Im w>0\}$. Then $\phi \circ F\left(z^{0}\right)=(0, \ldots, 0)$ and $\phi \circ F$ maps $U$ biholomorphically onto $\mathbf{H}^{n} \cap \phi \circ F(U)$. For $l=1, \ldots, n$ let

$$
\tau_{k}^{(l)}=\Re \phi_{l} \circ F\left(z^{k}\right), \quad \lambda_{k}^{(l)}=\Im \phi_{l} \circ F\left(z^{k}\right) .
$$

Then

is an automorphism of $\mathbf{H}^{n}$. Consider

$$
A^{k}(z):=\left(\frac{z_{1}-\tau_{k}^{(1)}}{\lambda_{k}^{(1)}}, \ldots, \frac{z_{n}-\tau_{k}^{(n)}}{\lambda_{k}^{(n)}}\right)
$$

$$
\Lambda^{k}:=\phi^{-1} \circ A^{k} \circ \phi \circ F: U \cap D \rightarrow \Delta^{n} .
$$


Then $\Lambda^{k}$ are biholomorphic imbeddings of $U \cap D$ into $\Delta^{n}$. Observe that

$$
\Lambda^{k}\left(z^{k}\right)=\phi^{-1} \circ A^{k}\left(\tau_{k}^{(1)}+\iota \lambda_{k}^{(1)}, \ldots, \tau_{k}^{(n)}+\iota \lambda_{k}^{(n)}\right)=(0, \ldots, 0) .
$$

Additionally, $\left\{\Lambda^{k}(U \cap D)\right\}$ is a sequence of subdomains of $\Delta^{n}$ that exhausts $\Delta^{n}$. Indeed, let $L$ be a compact subdomain of $\Delta^{n}$. Exploiting the continuity of the mapping $\phi \circ F$, we see that $\phi \circ F\left(z^{k}\right) \rightarrow 0$ as $k \rightarrow \infty$. In other words,

$$
\left(\tau_{k}^{(1)}+\iota \lambda_{k}^{(1)}, \ldots, \tau_{k}^{(n)}+\iota \lambda_{k}^{(n)}\right) \rightarrow(0, \ldots, 0)
$$

as $k \rightarrow \infty$. This gives that for each $l=1,2, \ldots, n$, both $\tau_{k}^{(l)} \rightarrow 0$ and $\lambda_{k}^{(l)} \rightarrow 0$ as $k \rightarrow \infty$. As a result, $\left(A^{k}\right)^{-1}$ maps $\phi(L)$ onto a neighbourhood $W \subset \phi \circ F(U \cap D)$ of $(0, \ldots, 0)$ in $\mathbf{H}^{n}$ for sufficiently large $k$. This is just the assertion that $L$ is compactly contained in $\Lambda^{k}(U \cap D)$ for all $k$ large. This finishes the proof of the claim. Now, fix $R>0$ and let $\theta: \Delta^{n} \rightarrow B_{\Delta^{n}}(0, R)$ be a biholomorphism. For $\epsilon>0$ given, there exists a $\delta \in(0,1)$ such that

$$
B_{\Delta^{n}}(0, R-\epsilon) \subset \theta\left(\Delta^{n}(0, \delta)\right) \subset B_{\Delta^{n}}(0, R) .
$$

Using lemma 2.3 in [14, we get that

$$
B_{\Delta^{n}}(0, R) \subset B_{\Lambda^{k}(U \cap D)}(0, R+\epsilon)
$$

for all $k$ large. Consequently,

$$
\theta\left(\Delta^{n}(0, \delta)\right) \subset B_{\Lambda^{k}(U \cap D)}(0, R+\epsilon) \subset \Lambda^{k}(U \cap D)
$$

for all $k$ large. It is already known that $\Lambda^{k}(U \cap D) \subset \Delta^{n}$, therefore

$$
B_{\Lambda^{k}(U \cap D)}(0, r) \subset B_{\Delta^{n}}(0, r)
$$

for all $r>0$ and all $k$. From (7.1), (7.2) and (7.3), we conclude that there exists a biholomorphic imbedding $\theta: \Delta^{n}(0, \delta) \rightarrow \Lambda^{k}(U \cap D)$ such that

$$
B_{\Lambda^{k}(U \cap D)}(0, R-\epsilon) \subset \theta\left(\Delta^{n}(0, \delta)\right)
$$

so that

$$
h_{\Lambda^{k}(U \cap D)}\left(0, \Delta^{n}\right) \leq(R-\epsilon)^{-1} .
$$

Since $R>0$ was arbitrary, we have $\lim _{k \rightarrow \infty} h_{\Lambda^{k}(U \cap D)}\left(0, \Delta^{n}\right)=0$. In addition,

$$
h_{\Lambda^{k}(U \cap D)}\left(0, \Delta^{n}\right)=h_{\Lambda^{k}(U \cap D)}\left(\Lambda^{k}\left(z^{k}\right), \Delta^{n}\right)=h_{U \cap D}\left(z^{k}, \Delta^{n}\right) .
$$

It follows that

$$
h_{U \cap D}\left(z^{k}, \Delta^{n}\right) \rightarrow 0
$$

as $k \rightarrow \infty$. Since $h$ can be localised near $z^{0}$ by proposition 3.6 we have

$$
h_{D}\left(z^{k}, \Delta^{n}\right) \rightarrow 0
$$

as $k \rightarrow \infty$.

\section{Applications}

In this section we collect several consequences of the results presented in the prequel. To begin with, we present an alternate proof of the following theorem of Wong-Rosay (39, 32]) using theorem 4.1 and characterise $\Delta^{n}$ in the class of generic analytic polyhedra. It must be mentioned that this is motivated by the main theorem of Kim and Pagano from [24] and in fact provides an alternate proof of their result in case the orbit accumulates at a singular boundary point. The question of recovering their full theorem using Fridman's invariant seems interesting.

Theorem 8.1. Let $D \subset \mathbf{C}^{n}$ be a bounded domain. Let $z^{0} \in \partial D$ have a neighbourhood $U$ such that $U \cap \partial D$ is $C^{2}$-smooth strongly pseudoconvex. If $z^{0}$ is an orbit accumulation point for the action of Aut $(D)$ on $D$, then $D$ is biholomorphically equivalent to $\mathbf{B}^{n}$. 
Proof. By hypothesis, there exists a sequence $\left\{F^{k}\right\} \subset \operatorname{Aut}(D)$ and $p^{0} \in D$ such that $F^{k}\left(p^{0}\right) \rightarrow z^{0} \in \partial D$ as $k \rightarrow \infty$. Then using theorem 4.1, we have

$$
h_{D}\left(F^{k}\left(p^{0}\right)\right) \rightarrow 0
$$

as $k \rightarrow \infty$. Since

$$
h_{D}\left(F^{k}\left(p^{0}\right)\right)=h_{F^{k}(D)}\left(F^{k}\left(p^{0}\right)\right)=h_{D}\left(p^{0}\right)
$$

for all $k$, it follows that $h_{D}\left(p^{0}\right)=0$. Applying proposition 3.3, we conclude that $D$ is biholomorphic to $\mathbf{B}^{n}$.

Theorem 8.2. Let $D \subset \mathbf{C}^{n}$ be a bounded domain. Let $z^{0} \in \partial D$ have a neighbourhood $U$ such that $U \cap \partial D=\left\{z \in \mathbf{C}^{n}|| f^{i}(z) \mid<1\right.$ for all $\left.1 \leq i \leq n\right\}$ and $\left|f^{i}\left(z^{0}\right)\right|=1$ for all $1 \leq i \leq n$ where $f^{i} \in$ $\mathcal{O}(U)$ for all $1 \leq i \leq n$. Assume that $d f^{1} \wedge \ldots \wedge d f^{n} \neq 0$ at $z^{0}$. If $z^{0}$ is an orbit accumulation point for the action of $A u t(D)$ on $D$, then $D$ is biholomorphically equivalent to $\Delta^{n}$.

Proof. By hypothesis, there exists a sequence $\left\{F^{k}\right\} \subset \operatorname{Aut}(D)$ and $p^{0} \in D$ such that $F^{k}\left(p^{0}\right) \rightarrow z^{0} \in \partial D$ as $k \rightarrow \infty$. It follows from theorem 7.1 that

$$
h_{D}\left(F^{k}\left(p^{0}\right), \Delta^{n}\right) \rightarrow 0
$$

as $k \rightarrow \infty$. Since

$$
h_{D}\left(F^{k}\left(p^{0}\right), \Delta^{n}\right)=h_{F^{k}(D)}\left(F^{k}\left(p^{0}\right), \Delta^{n}\right)=h_{D}\left(p^{0}, \Delta^{n}\right)
$$

for all $k$, we infer that $h_{D}\left(p^{0}, \Delta^{n}\right)=0$. Applying proposition 3.3, we conclude that $D$ is biholomorphic to $\Delta^{n}$.

As a consequence of theorem [5.1, it is also possible to recover parts of the main theorem of [10, the emphasis here being a different approach - wherein there is no need apriori to establish the boundary distance estimate.

Theorem 8.3. A strongly pseudoconvex domain with $C^{2}$-smooth boundary cannot be mapped biholomorphically onto a smoothly bounded weakly pseudoconvex domain of finite type in $\mathbf{C}^{2}$.

Proof. Suppose there exists a biholomorphism $f$ from a strongly pseudoconvex domain $D_{1}$ onto a weakly pseudoconvex domain $D_{2}$ of finite type in $\mathbf{C}^{2}$. Let $q^{0} \in \partial D_{2}$ be a weakly pseudoconvex boundary point. Then there exists a local coordinate system in a neighbourhood of $q^{0}$ taking $q^{0}$ to the origin such that the domain $D_{2}$ near origin can be written as

$$
\left\{\left(z_{1}, z_{2}\right) \in \mathbf{C}^{2}: 2 \Re z_{2}+H_{2 m}\left(z_{1}, \bar{z}_{1}\right)+o\left(|z|^{2 m}+\Im z_{2}\right)<0\right\}
$$

where $H_{2 m}$ is a homogeneous subharmonic polynomial of degree $2 m \geq 2$ in $z_{1}$ and $\bar{z}_{1}$ which does not contain any harmonic terms. Let $\left\{q^{j}\right\}$ be a sequence of points in $D_{2}$ converging to $q^{0}$ normally, i.e.,

$$
q^{j}=q^{0}-d\left(q^{j}, \partial D_{2}\right) n\left(q^{0}\right)
$$

where $n\left(q^{0}\right)$ denotes the unit outward normal to $\partial D_{2}$ at $q^{0}$. Choose points $\left\{p^{j}\right\} \subset D_{1}$ such that $f\left(p^{j}\right)=q^{j}$. Then for any $x \in D_{1}$, we have

$$
d_{D_{1}}\left(x, p^{j}\right)=d_{D_{2}}\left(f(x), q^{j}\right) .
$$

The above observation together with the completeness of $D_{1}$ and $D_{2}$ implies that the sequence $\left\{p^{j}\right\}$ must cluster on $\partial D_{1}$. Hence, we may assume that the sequence $\left\{p^{j}\right\}$ converges to $p^{0} \in \partial D_{1}$. It follows from the biholomorphic invariance of the function $h$ that

$$
h_{D_{2}}\left(q^{j}\right)=h_{D_{1}}\left(p^{j}\right) .
$$

Applying theorem 4.1 and theorem [5.1, we get after letting $j \rightarrow \infty$ that

$$
h_{D_{2, \infty}}((0,-1))=0 \text {. }
$$

Here $D_{2, \infty}$ is the limiting domain obtained by scaling $D_{2}$ with respect to the base point $q^{0}$ and the sequence $\left\{q^{j}\right\}$. More concretely,

$$
D_{2, \infty}=\left\{\left(z_{1}, z_{2}\right) \in \mathbf{C}^{2}: 2 \Re z_{2}+H_{2 m}\left(z_{1}, \bar{z}_{1}\right)<0\right\} .
$$

Now, using proposition 3.3, we infer from (8.1) that $D_{2, \infty}$ is biholomorphically equivalent to $\mathbf{B}^{2}$. Let $\tilde{f}$ denote the biholomorphism from the domain $D_{2, \infty}$ to $\mathbf{B}^{2}$. Then for any point $\left(0, \iota a^{0}\right), a^{0} \in \mathbf{R}$ and any 
sequence of points $\left\{z^{j}\right\} \subset D_{2, \infty}$ converging to $(0, \iota a) \in \partial D_{2, \infty}$, the corresponding image sequence $\left\{\tilde{f}\left(z^{j}\right)\right\}$ clusters at some point of $\partial \mathbf{B}^{2}$. Composing with an appropriate Cayley transform, we may assume that there exists a biholomorphism $F$ from $D_{2, \infty}$ onto the unbounded realization of the unit ball, namely to

$$
\Sigma=\left\{z \in \mathbf{C}^{2}: 2 \Re z_{2}+\left|z_{1}\right|^{2}<0\right\}
$$

with the property that the cluster set of $F$ at the point $\left(0, \iota a^{0}\right) \in \partial D_{2, \infty}$ contains a boundary point of $\Sigma$ different from the point at infinity on $\partial \Sigma$. Then it follows from theorem 2.1 of 8 that $F$ extends holomorphically to a neighbourhood of $\left(0, \iota a^{0}\right)$. Since $D_{2, \infty}$ is invariant under translations in the imaginary $z_{2}$ - direction, we can assume that $\left(0, \iota a^{0}\right)=(0,0)$. This shows that $F$ extends holomorphically to a neighbourhood of the origin. In addition, by composing with an automorphism of $\Sigma$, if necessary we may assume that $F$ takes the origin to the origin. Now, we intend to use the arguments from [10] to show that $H_{2 m}\left(z_{1}, \bar{z}_{1}\right) \equiv\left|z_{1}\right|^{2}$. Since $F$ extends holomorphically near the origin, we can write

$$
\Re\left(F_{2}(z)\right)+\left|F_{1}(z)\right|^{2}=h(z)\left(\Re z_{2}+H_{2 m}\left(z_{1}, \bar{z}_{1}\right)\right)
$$

in a neighbourhood of the origin where $h$ is a real analytic function near the origin and $h(0,0) \neq 0$. Now let

$$
F_{1}\left(z_{1}, 0\right)=\sum_{\mu \geq s} b_{\mu} z_{1}^{\mu}
$$

where $s \geq 1$. Also, $F_{2}(z)=\beta z_{2}+o(|z|)$ where $\beta \in \mathbf{R} \backslash\{0\}$. Indeed, applying Hopf's lemma to the function $\Re F_{2}$, we see that $\partial\left(\Re F_{2}\right) / \partial x_{2}(0)>0$ where $x_{2}=\Re z_{2}$. Furthermore, since $F:\left(\partial D_{2, \infty},(0,0)\right) \rightarrow$ $(\partial \Sigma,(0,0))$ and $F$ extends holomorphically near the origin, it takes the complex normal to $\partial D_{2, \infty}$ at the origin to the complex normal to $\partial \Sigma$ at the origin. In particular, $\partial F_{2} / \partial z_{1} \equiv 0$. Now, setting $z_{2}=0$ in (8.2) yields

$$
\Re\left(o\left(\left|z_{1}\right|\right)\right)+b_{s} \bar{b}_{s}\left|z_{1}\right|^{2 s}+o\left(\left|z_{1}\right|^{2 s}\right)=h\left(z_{1}, 0\right) H_{2 m}\left(z_{1}, \bar{z}_{1}\right) .
$$

Since $H_{2 m}$ does not contain any harmonic terms, we have

$$
b_{s} \bar{b}_{s}\left|z_{1}\right|^{2 s}+o\left(\left|z_{1}\right|^{2 s}\right)=h(0,0) H_{2 m}\left(z_{1}, \bar{z}_{1}\right) \text {. }
$$

Since the polynomial $H_{2 m}$ is homogeneous of degree $2 m$, we conclude that $2 m=2 s$ and $H_{2 m}\left(z_{1}, \bar{z}_{1}\right)=$ $\left|z_{1}\right|^{2 m}$. It follows that $\mathbf{B}^{2} \simeq D_{2, \infty} \simeq\left\{\left(z_{1}, z_{2}\right) \in \mathbf{C}^{2}: 2 \Re z_{2}+\left|z_{1}\right|^{2 m}<0\right\} \simeq \tilde{D}=\left\{\left(z_{1}, z_{2}\right) \in \mathbf{C}^{2}\right.$ : $\left.\left|z_{1}\right|^{2 m}+\left|z_{2}\right|^{2}<1\right\}$. Let $G: \mathbf{B}^{2} \rightarrow \tilde{D}$ be the biholomorphism which in addition may be assumed to presume the origin. Since $\mathbf{B}^{2}$ and $\tilde{D}$ are both circular domains, it follows that $G$ is linear. This forces that $2 m=2$ and hence $H_{2 m}\left(z_{1}, \bar{z}_{1}\right)=\left|z_{1}\right|^{2}$.

But this exactly means that there exists a local coordinate system in a neighbourhood of $q^{0}$ which takes the point $q^{0}$ to the origin and the domain $D_{2}$ near the origin can be written as

$$
\left\{\left(z_{1}, z_{2}\right) \in \mathbf{C}^{2}: 2 \Re z_{2}+\left|z_{1}\right|^{2}+o\left(\left|z_{1}\right|^{2}+\Im z_{2}\right)<0\right\}
$$

This contradicts the fact that $q^{0}=(0,0)$ is a weakly pseudoconvex point and proves the theorem.

The local version of the preceding theorem can also be recovered similarly:

Theorem 8.4. Let $D_{1}$ and $D_{2}$ be two domains in $\mathbf{C}^{2}$. Assume that $\partial D_{1}$ is $C^{2}$-smooth strongly pseudoconvex in a neighbourhood of a point $p^{0} \in \partial D_{1}$. Suppose that $\partial D_{2}$ is $C^{\infty}{ }_{\text {-smooth weakly pseudoconvex of }}$ finite type in a neighbourhood of a point $q^{0} \in \partial D_{2}$. Then there cannot be a biholomorphism $f$ from $D_{1}$ onto $D_{2}$ with the property that $q^{0}$ belongs to the cluster set of $f$ at $p^{0}$.

If such a biholomorphism $f$ exists, the first step in proving the above result is to establish that $f$ extends to continuous mapping on a neighbourhood of $p^{0}$ in $\bar{D}_{1}$. This requires the fact that $f$ decreases the distance to the boundary, which is a consequence of well known estimates for the Kobayashi metric. This is the content of proposition 2.1 of [10. Scaling the domain $D_{2}$ with respect a sequence converging normally to $q^{0}$ and proceeding as in the proof of theorem 8.3. we obtain that $D_{2}$ near the $q^{0}=(0,0)$ is given by

$$
\left\{\left(z_{1}, z_{2}\right) \in \mathbf{C}^{2}: 2 \Re z_{2}+\left|z_{1}\right|^{2}+o\left(\left|z_{1}\right|^{2}+\Im z_{2}\right)<0\right\}
$$

which leads to a contradiction.

Using similar ideas, the following theorem of Coupet-Gaussier-Sukhov ([7]) can also be recovered: 
Theorem 8.5. A strongly pseudoconvex domain with $C^{2}$-smooth boundary cannot be mapped biholomorphically onto a smoothly bounded convex (but not strongly pseudoconvex) domain of finite type in $\mathbf{C}^{n}(n>1)$.

Proof. Suppose there exists a biholomorphism $f$ from a $C^{2}$-smooth strongly pseudoconvex domain $D_{1}$ onto a smoothly bounded convex but not strongly pseudoconvex domain $D_{2}$ of finite type in $\mathbf{C}^{n}$. Let $q^{0} \in \partial D_{2}$. By [40] there exists a local coordinate system in a neighbourhood of $q^{0}$ taking $q^{0}$ to the origin such that the domain $D_{2}$ near origin can be written as

$$
\left\{\left({ }^{\prime} z, z_{n}\right) \in \mathbf{C}^{n}: 2 \Re z_{n}+P_{0}\left({ }^{\prime} z\right)+R(z)<0\right\}
$$

where $P_{0}$ is a nondegenerate weighted homogeneous polynomial of degree 1 with respect to the weights $\mathcal{M}(\partial D, 0)$ and $R$ denotes terms of degree at least two. Scaling the domain $D_{2}$ with respect to a sequence converging normally to the point $q^{0}$ as in the proof of theorem 8.3. we infer that $D_{2, \infty}$ is biholomorphically equivalent to $\mathbf{B}^{n}$. Here $D_{2, \infty}$ denotes the limiting domain obtained by scaling $D_{2}$ :

$$
D_{2, \infty}=\left\{\left({ }^{\prime} z, z_{n}\right) \in \mathbf{C}^{n}: 2 \Re z_{n}+P_{0}\left({ }^{\prime} z\right)<0\right\} .
$$

Then as before, we may assume that for any point $\left(0, \iota a^{0}\right), a^{0} \in \mathbf{R}$ there exists a biholomorphism $F$ from $D_{2, \infty}$ onto the unbounded realization of the unit ball, namely to

$$
\Sigma=\left\{z \in \mathbf{C}^{n}: 2 \Re z_{n}+\left.\left.\right|^{\prime} z\right|^{2}<0\right\}
$$

with the property that the cluster set of $F$ at the point $\left({ }^{\prime} 0, \iota a^{0}\right) \in \partial D_{2, \infty}$ contains a point $\zeta^{0} \in \partial \Sigma$ where $\zeta^{0}$ is different from the point at infinity on $\partial \Sigma$. Applying theorem 2.1 of [8], we get that $F$ extends holomorphically to a neighbourhood $U$ of $\left({ }^{\prime} 0, \iota a^{0}\right)$. We now claim that $F$ extends biholomorphically near $\left({ }^{\prime} 0, \iota a^{0}\right)$. To see this, denote by $l=\left\{\left({ }^{\prime} 0, \iota a\right) \in \mathbf{C}^{n}: a \in \mathbf{R}\right\} \subset \partial D_{2, \infty}$. Assume on the contrary that the Jacobian of $F$ vanishes identically on $U \cap l$. In that case the Jacobian of $F$ vanishes on the entire $z_{n}$ axis which intersects the domain. This violates the fact that $F$ is injective on $D_{2, \infty}$. This contradiction completes the proof of the claim. Now, pick $\left({ }^{\prime} 0, \iota \tilde{a}\right) \in U \cap l$ such that the Jacobian of $F$ does not vanish at $\left({ }^{\prime} 0, \iota \tilde{a}\right)$. Consequently, $F$ extends biholomorphically past $\left({ }^{\prime} 0, \iota \tilde{a}\right) \in \partial D_{2, \infty}$. Since $D_{2, \infty}$ is invariant under translations in the imaginary $z_{n^{-}}$direction, we can assume that $\left({ }^{\prime} 0, \iota \tilde{a}\right)=\left({ }^{\prime} 0,0\right)$. This shows that $F$ extends biholomorphically to a neighbourhood of the origin. In addition, by composing with an automorphism of $\Sigma$, if necessary we may assume that $F$ maps the origin to the origin. Since the Levi-form is preserved under local biholomorphisms around a boundary point, this forces that $\left({ }^{\prime} 0,0\right) \in \partial D_{2, \infty}$ be strongly pseudoconvex. This contradiction proves the theorem.

A local version of this theorem also holds:

Theorem 8.6. Let $D_{1}$ and $D_{2}$ be domains in $\mathbf{C}^{n}$. Assume that $\partial D_{1}$ is $C^{2}$-smooth strongly pseudoconvex in a neighbourhood of a point $p^{0} \in \partial D_{1}$ and that $\partial D_{2}$ is $C^{\infty}$-smooth convex (but not strongly pseudoconvex) of finite type in a neighbourhood of a point $q^{0} \in \partial D_{2}$. Then there cannot be a biholomorphism $f$ from $D_{1}$ onto $D_{2}$ with the property that $q^{0}$ belongs to the cluster set of $f$ at $p^{0}$.

Suppose that such a $f$ exists. Then proposition 2.1 of [10] implies that $f$ extends continuously near $p^{0}$. Now, scaling the domain $D_{2}$ with respect to a sequence converging normally to $q^{0}$ and arguing as in the proof of theorem 8.5 we obtain that the domain $D_{2}$ is strongly psequdoconvex near $q^{0}$, thereby leading to a contradiction.

Next, we apply the invariance property of the function $h$ to deduce the biholomorphic inequivalence of a bounded domain with smooth and piecewise smooth boundaries.

A domain $D \subset \mathbf{C}^{n}$ is said to have piecewise $C^{r}$-smooth boundary if there are real-valued functions $\rho_{i}, i=1, \ldots, m$, in a neighbourhood $V$ of the closure of $D$ satisfying the following conditions:

- $\partial D \subset \bigcup_{i=1}^{m}\left\{z \in V: \rho_{i}(z)=0\right\}$

- For every subset $\left\{i_{1}, \ldots, i_{k}\right\} \subset\{1, \ldots, m\}$ the form $\partial \rho_{i_{1}} \wedge \ldots \wedge \partial \rho_{i_{k}} \neq 0$ on the intersection $\bigcap_{i=1}^{m}\left\{z \in V: \rho_{i}(z)=0\right\}$

The latter condition means that $\left\{z \in V: \rho_{i}(z)=0\right\}$ is a $C^{r}$-smooth hypersurface in general position. 
Theorem 8.7. Let $D_{1}$ and $D_{2}$ be two bounded domains in $\mathbf{C}^{n}(n>1), D_{1}$ having strongly pseudoconvex boundary of class $C^{2}$ and $D_{2}$ having piecewise $C^{2}$-smooth, but not smooth boundary. Then $D_{1}$ and $D_{2}$ are biholomorphically inequivalent.

Proof. Suppose there exists a biholomorphism $f: D_{1} \rightarrow D_{2}$. Then the strong pseudoconvexity of $D_{1}$ implies that $D_{2}$ is pseudoconvex. Then from the results of 30 there follows the existence of a point $q^{0} \in \partial D_{2}$ (for definiteness we shall assume $q^{0}$ is the origin) and $C^{2}$-smooth real-valued functions $\rho_{i}, i=1, \ldots, k(2 \leq k \leq n)$ defined in some neighbourhood $U$ of the origin such that:

(i) $\left({ }^{\prime} 0,0\right) \in \partial D_{2} \cap\left\{z \in U: \rho_{i}(z)=0, i=1,2, \ldots, k\right\}$

(ii) $D_{2} \cap U=\left\{z \in U: \rho_{i}(z)<0, i=1,2, \ldots, k\right\}$

(iii) $\bar{\partial} \rho_{1} \wedge \ldots \wedge \bar{\partial} \rho_{k}(z) \neq 0$ for $z \in U$,

(iv) for some $A>0$ the function $\rho=\sum_{i=1}^{k} \rho_{i}+A \sum_{i=1}^{k} \rho_{i}^{2}$ is strictly plurisubharmonic in $U$ and $D_{2} \cap U \subset$ $\{z \in U: \rho(z)<0\}$.

Scaling the domain $D_{2}$ as in [31] with respect to a sequence converging to the point $q^{0}$ and using the Fridman's invariant as before, we infer that the limiting domain $D_{2, \infty}$ is biholomorphic to $\mathbf{B}^{n}$. It turns out (for details see [31]) that $D_{2, \infty}$ is biholomorphic to a product of balls $\mathbf{B}^{n_{1}} \times \ldots \times \mathbf{B}^{n_{k}}$ where $\mathbf{B}^{n_{j}}=\left\{z \in \mathbf{C}^{n_{j}}:|z|<1\right\}$ and $k \geq 2$. This shows that $\mathbf{B}^{n}$ is biholomorphic to a product of balls which is a contradiction. Hence the result follows.

In general, it is very difficult to provide an explicit expression for the Kobayashi distance between two points of a given domain. the most that can be done is to describe the Kobayashi distance in terms of Euclidean parameters such as the distance to the boundary. For instance, it is known that if $D$ is a bounded strongly pseudoconvex domain with $C^{2}$-smooth boundary, then for all $R>0$, there exists positive constants $c_{i}$ and $C_{i}(i=1,2)$ depending only on $R$ and $D$ such that for each $q$ in $D$ sufficiently close to $\partial D$

$$
P\left(q ; c_{1} d(q, \partial D), c_{2} d(q, \partial D)^{1 / 2}\right) \subset B_{D}(q, r) \subset P\left(q ; C_{1} d(q, \partial D), C_{2} d(q, \partial D)^{1 / 2}\right)
$$

where $P\left(q ; r_{1}, r_{2}\right)$ denotes the polydisc centered at $q$ with radius $r_{1}$ in the complex normal direction and the radius $r_{2}$ in each complex tangential direction.

These estimates on the unit ball in $\mathbf{C}^{n}$ can be obtained by direct computation using the explicit formula for the Kobayashi distance on $\mathbf{B}^{n}$. For a strongly pseudoconvex domain $D$, one has to work with suitable ellipsoids tangent to $\partial D$ to get these results.

This approach will not work for weakly pseudoconvex domains of finite type in $\mathbf{C}^{2}$ because of nonavailability of a suitable model domain. Instead, we make use of the rescaling technique given in [5].

Proposition 8.8. Let $D$ be a bounded weakly pseudoconvex domain of finite type in $\mathbf{C}^{2}$ with $C^{\infty}$ - smooth boundary. Then for all $R>0$, there exist positive constants $C_{1}$ and $C_{2}$ depending only on $R$ and $D$ such that for each $q$ in $D$ sufficiently close to $\partial D$

$$
Q\left(q, C_{1} d(q, \partial D)\right) \subset B_{D}(q, R) \subset Q\left(q, C_{2} d(q, \partial D)\right)
$$

where $Q(q, r)$ denotes the Catlin's bidisc.

Proof. Firstly, observe that for each $q$ in $D$, we can always find constants $C_{i}(q, R), i=1,2$ such that

$$
Q\left(q, C_{1}(q, R) d(q, \partial D)\right) \subset B_{D}(q, R) \subset Q\left(q, C_{2}(q, R) d(q, \partial D)\right)
$$

by virtue of the following fact: the Kobayashi ball $B_{D}(q, R)$ and the pseudo-bidisc $Q(q, d(q, \partial D))$ are both open sets. The above proposition asserts that the constants can be chosen independent of $q$.

To establish that $Q\left(q, C_{1} d(q, \partial D)\right) \subset B_{D}(q, R)$ for some uniform constant $C_{1}$, suppose that this is not true. Then there exists a sequence of points $\left\{q^{j}\right\} \subset D$ converging to some point $q^{0} \in \partial D$ and a sequence of positive real numbers $C_{j} \rightarrow 0$ as $j \rightarrow \infty$ such that

$$
Q\left(q^{j}, C_{j} d\left(q^{j}, \partial D\right)\right) \nsubseteq B_{D}\left(q^{j}, R\right) .
$$


Assume that $q^{0}=(0,0)$ without loss of generality. Let $D=\{\rho(z, \bar{z})<0\}$ where $\rho$ is a smooth defining function for $\partial D$ which has the form

$$
\rho(z, \bar{z})=2 \Re z_{2}+H_{2 m}\left(z_{1}, \bar{z}_{1}\right)+o\left(|z|^{2 m}+\Im z_{2}\right)
$$

near the origin, with $H_{2 m}$ a homogeneous subharmonic polynomial of degree $2 m \geq 2$ in $z_{1}$ and $\bar{z}_{1}$ which does not contain any harmonic terms. Pick points $\zeta^{j} \in \partial D$ be closest to $q^{j}$. To be more precise, $\zeta^{j}=q^{j}+\left(0, \epsilon_{j}\right), \epsilon_{j}>0$. Choose points $p^{j} \in Q\left(q^{j}, C_{j} d\left(q^{j}, \partial D\right)\right)$ such that $d_{D}\left(p^{j}, q^{j}\right)=R$. We now scale the domain $D$ with respect to the base point $q^{0}=(0,0)$ and the sequence $\left\{q^{j}\right\}$. Recall that

$$
\begin{aligned}
& \Delta_{\zeta^{j}}^{\epsilon_{j}} \circ \phi^{\zeta^{j}}\left(Q\left(q^{j}, C_{j} d\left(q^{j}, \partial D\right)\right)\right)= \\
& \Delta_{\zeta^{j}}^{\epsilon_{j}} \circ \phi^{\zeta^{j}} \circ\left(\phi^{q^{j}}\right)^{-1}\left(\Delta\left(0, \tau\left(q^{j}, C_{j} d\left(q^{j}, \partial D\right)\right)\right) \times \Delta\left(0, C_{j} d\left(q^{j}, \partial D\right)\right)\right)= \\
& \left\{w:\left|w_{1}\right|<\frac{\tau\left(q^{j}, C_{j} d\left(q^{j}, \partial D\right)\right)}{\tau\left(\zeta^{j}, \epsilon_{j}\right)},\left|\epsilon_{j}^{-1}\left(\sum_{l=1}^{m} \alpha^{l, j} w_{1}^{l}\right)+d^{0}\left(\zeta^{j}\right) w_{2}+1\right|<\frac{C_{j} d\left(q^{j}, \partial D\right) d^{0}\left(q^{j}\right)}{\epsilon_{j}}\right\}
\end{aligned}
$$

where

$$
\alpha^{l, j}=\left(d^{l}\left(\zeta^{j}\right)-d^{l}\left(q^{j}\right)\right) \tau\left(\zeta^{j}, \epsilon_{j}\right)^{l}
$$

Among other things, the following claims were proved in [5].

- $\tau\left(q^{j}, C_{j} d\left(q^{j}, \partial D\right)\right) \lesssim C_{j} \tau\left(\zeta^{j}, \epsilon_{j}\right)$,

- $\left|d^{l}\left(q^{j}\right)\right| \lesssim \epsilon^{j}\left(\tau\left(\zeta^{j}, \epsilon_{j}\right)\right)^{-l}$,

- $\left|d^{l}\left(\zeta^{j}\right)\right| \lesssim \epsilon^{j}\left(\tau\left(\zeta^{j}, \epsilon_{j}\right)\right)^{-l}$,

- $d^{0}\left(\zeta^{j}\right) \approx 1$ and $d^{0}\left(q^{j}\right) \approx 1$.

Moreover, $d\left(q^{j}, \partial D\right) \approx \epsilon_{j}$. These estimates together with the explicit description of the set

$$
\Delta_{\zeta^{j}}^{\epsilon_{j}} \circ \phi^{\zeta^{j}}\left(Q\left(q^{j}, C_{j} d\left(q^{j}, \partial D\right)\right)\right)
$$

show that the sequence $\left\{\Delta_{\zeta^{j}}^{\epsilon_{j}} \circ \phi^{\zeta^{j}}\left(p^{j}\right)\right\}$ is bounded. Since $C_{j} \rightarrow 0$, it follows that

$$
\Delta_{\zeta^{j}}^{\epsilon_{j}} \circ \phi^{\zeta^{j}}\left(p^{j}\right) \rightarrow(0,-1)
$$

as $j \rightarrow \infty$. The scaled maps $\Delta_{\zeta^{j}}^{\epsilon_{j}} \circ \phi^{\zeta^{j}}$ are biholomorphisms and hence Kobayashi isometries from $D$ onto the dilated domains $D^{j}:=\left\{z \in \mathbf{C}^{2}: \rho \circ\left(\phi^{\zeta^{j}}\right)^{-1} \circ\left(\Delta_{\zeta^{j}}^{\epsilon_{j}}\right)^{-1}(z)<0\right\}$. Therefore,

$$
d_{D^{j}}\left(\Delta_{\zeta^{j}}^{\epsilon_{j}} \circ \phi^{\zeta^{j}}\left(p^{j}\right), \Delta_{\zeta^{j}}^{\epsilon_{j}} \circ \phi^{\zeta^{j}}\left(q^{j}\right)\right)=R
$$

which exactly means that

$$
d_{D^{j}}\left(\Delta_{\zeta^{j}}^{\epsilon_{j}} \circ \phi^{\zeta^{j}}\left(p^{j}\right),\left(0,-1 / d^{0}\left(\zeta^{j}\right)\right)\right)=R .
$$

Note that $\left(0,-1 / d^{0}\left(\zeta^{j}\right)\right) \rightarrow(0,-1)$ as $j \rightarrow \infty$. Applying lemma [5.7 we get after letting $j \rightarrow \infty$ that

$$
d_{D_{\infty}}((0,-1),(0,-1))=R
$$

where $D_{\infty}$ is the limiting domain obtained by scaling $D$ with respect to the base point $q^{0}$ and the sequence $\left\{q^{j}\right\}$. This contradiction proves one part of the desired estimate.

We now show that $B_{D}(q, R) \subset Q\left(q, C_{2} d(q, \partial D)\right)$ for some constant $C_{2}$ independent of $q$. Suppose this is not true. Then there exist a sequence of points $\left\{q^{j}\right\} \subset D$ converging to some point $q^{0} \in \partial D$ and a sequence of positive real numbers $C_{j} \rightarrow \infty$ as $j \rightarrow \infty$ such that

$$
Q\left(q^{j}, C_{j} d\left(q^{j}, \partial D\right)\right) \nsupseteq B_{D}\left(q^{j}, R\right) .
$$

Assume that $q^{0}=(0,0)$ without loss of generality. Choose $\zeta^{j} \in \partial D$ be closest to $q^{j}$ and points $p^{j}$ in the complement of the closure of $Q\left(q^{j}, C_{j} d\left(q^{j}, \partial D\right)\right)$ such that $p^{j} \in B_{D}\left(q^{j}, R\right)$. Then for $\epsilon_{j}, D^{j}$ and $D_{\infty}$ defined analogously, we have

$$
d_{D^{j}}\left(\Delta_{\zeta^{j}}^{\epsilon_{j}} \circ \phi^{\zeta^{j}}\left(p^{j}\right), \Delta_{\zeta^{j}}^{\epsilon_{j}} \circ \phi^{\zeta^{j}}\left(q^{j}\right)\right)<R .
$$


Said differently,

$$
d_{D^{j}}\left(\Delta_{\zeta^{j}}^{\epsilon_{j}} \circ \phi^{\zeta^{j}}\left(p^{j}\right),\left(0,-1 / d^{0}\left(\zeta^{j}\right)\right)\right)<R .
$$

Fix $\epsilon>0$ arbitrarily small and write $\left(0,-1 / d^{0}\left(\zeta^{j}\right)\right)=z^{j}$ and $(0,-1)=z^{0}$ for brevity. It now follows that

$$
\begin{aligned}
d_{D^{j}}\left(\Delta_{\zeta^{j}}^{\epsilon_{j}} \circ \phi^{\zeta^{j}}\left(p^{j}\right), z^{0}\right) & \leq d_{D^{j}}\left(\Delta_{\zeta^{j}}^{\epsilon_{j}} \circ \phi^{\zeta^{j}}\left(p^{j}\right), z^{j}\right)+d_{D^{j}}\left(z^{j}, z^{0}\right) \\
& \leq R+\epsilon
\end{aligned}
$$

for all $j$ large, where the second inequality uses the following consequence of lemma 5.7

$$
\lim _{j \rightarrow \infty} d_{D^{j}}\left(z^{j}, z^{0}\right)=d_{D_{\infty}}\left(z^{0}, z^{0}\right)=0 .
$$

It now follows from lemma 5.9 that

$$
\Delta_{\zeta^{j}}^{\epsilon_{j}} \circ \phi^{\zeta^{j}}\left(p^{j}\right) \in B_{D^{j}}\left(z^{0}, R+\epsilon\right) \subset B_{D_{\infty}}\left(z^{0}, R+2 \epsilon\right)
$$

for all $j$ sufficiently large. As a consequence, for all $j$ large

$$
d_{D_{\infty}}\left(\Delta_{\zeta^{j}}^{\epsilon_{j}} \circ \phi^{\zeta^{j}}\left(p^{j}\right), z^{0}\right)<R+2 \epsilon
$$

On the other hand,

$$
\left|\left(\Delta_{\zeta^{j}}^{\epsilon_{j}} \circ \phi^{\zeta^{j}}\left(p^{j}\right)\right)_{1}\right| \geq \frac{\tau\left(q^{j}, C_{j} d\left(q^{j}, \partial D\right)\right)}{\tau\left(\zeta^{j}, \epsilon_{j}\right)} \gtrsim C_{j} .
$$

Since $C_{j} \rightarrow \infty$ as $j \rightarrow \infty$, we conclude that

$$
\left|\left(\Delta_{\zeta^{j}}^{\epsilon_{j}} \circ \phi^{\zeta^{j}}\left(p^{j}\right)\right)_{1}\right| \rightarrow+\infty
$$

as $j \rightarrow \infty$. Consequently,

$$
\left|\Delta_{\zeta^{j}}^{\epsilon_{j}} \circ \phi^{\zeta^{j}}\left(p^{j}\right)-(0,-1)\right| \rightarrow+\infty
$$

as $j \rightarrow \infty$. This is not possible in view of (8.3). Hence the result.

It turns out that proceeding exactly as in the weakly pseudoconvex case using the scaling methods described in section 6 one can prove the following statement:

Proposition 8.9. Let $D$ be a smoothly bounded convex domain of finite type in $\mathbf{C}^{n}$. Then for all $R>0$, there exists positive constants $C_{1}$ and $C_{2}$ depending only on $R$ and $D$ such that for each $q$ in $D$ sufficiently close to $\partial D$

where $P(q, r)$ is as in (6.1).

$$
P\left(q, C_{1} d(q, \partial D)\right) \subset B_{D}(q, R) \subset P\left(q, C_{2} d(q, \partial D)\right)
$$

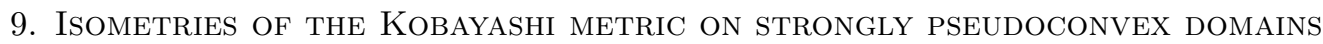

Let $D$ be a bounded strongly pseudoconvex domain in $\mathbf{C}^{n}$ with a $C^{2}$-smooth defining function $\rho^{0}$ defined on a neighbourhood $U$ of the closure of $D$. Choose $\rho^{k} \in C^{2}(U ; \mathbf{R})$ such that $\rho^{k}$ converges to $\rho^{0}$ in the $C^{2}$-topology, i.e.,

$$
\left\|\rho^{k}-\rho^{0}\right\|_{C^{2}(U)}=\sum_{j=1}^{n} \sup _{z \in U,|\alpha| \leq 2}\left|D^{\alpha}\left(\rho_{j}^{k}(z)-\rho_{j}^{0}(z)\right)\right| \rightarrow 0
$$

as $k \rightarrow \infty$. Setting $D^{k}=\left\{z \in U: \rho^{k}(z)<0\right\}$, observe that there exist uniform positive constants $C_{1}$ and $C_{2}$ such that for every $z$ in $N_{\epsilon}(\partial D)$, an $\epsilon$-neighbourhood of $\partial D$,

$$
C_{1} d(z, \partial D) \leq d\left(z, \partial D^{k}\right) \leq C_{2} d(z, \partial D) .
$$

where $d(\cdot, \partial D)$ is the Euclidean distance to the boundary.

Proposition 9.1. Let $D$ and $D^{k}$ 's be as described above. Let $q^{0}$ and $q^{1}$ be two distinct boundary points of $D$. Then for a suitable uniform constant $C$ and for all $k$ large,

$$
d_{D^{k}}(a, b) \geq-(1 / 2) \log d\left(a, \partial D^{k}\right)-(1 / 2) \log d\left(b, \partial D^{k}\right)-C
$$

whenever $a, b \in D$, $a$ is near $q^{0}$ and $b$ is near $q^{1}$. 
In case $a, b$ are close to the same boundary point, the upper estimate due to Forstneric and Rosay also remains stable under small $C^{2}$ perturbations.

Proposition 9.2. Let $D$ and $D^{k}$ 's be as described above and let $q^{0} \in \partial D$. Then there exists a neighbourhood $V=V\left(q^{0}\right)$ and a uniform constant $C>0$ such that

$$
\begin{aligned}
d_{D^{k}}(a, b) \leq-(1 / 2) \log d\left(a, \partial D^{k}\right)+(1 / 2) \log & \left(d\left(a, \partial D^{k}\right)+|a-b|\right) \\
& +(1 / 2) \log \left(d\left(b, \partial D^{k}\right)+|a-b|\right)-(1 / 2) \log d\left(b, \partial D^{k}\right)+C
\end{aligned}
$$

for all $a, b \in V \cap D$ and for all $k$ large.

The proof of proposition 9.2 will be given after lemma 9.8 and does not use any of the arguments presented till then. We proceed with the proof of proposition 9.1 first. This will need several steps. To begin with, we need to localise the Kobayashi metric. The following assertion about local peak functions will be useful.

Lemma 9.3. There exist uniform positive constants $C_{1}, C_{2}$ and $r$ such that for every $A^{k} \in \partial D^{k}$, if $\zeta \in B\left(A^{k}, r\right) \cap \partial D^{k}$ then there exists a local peak function $P_{\zeta}$ at $\zeta, P_{\zeta} \in \mathcal{O}\left(D_{1}^{k}\right) \cap C\left(\overline{D_{1}^{k}}\right)$ where $D_{1}^{k}=$ $B\left(A^{k}, r\right) \cap D^{k}$ is a neighbourhood of $A^{k}$ of uniform size with the property that

$$
C_{1}\left|1-P_{\zeta}(z)\right| \leq|z-\zeta| \leq C_{2} \sqrt{\left|1-P_{\zeta}(z)\right|}
$$

for all $z \in D_{1}^{k}$.

Proof. Note that the local peak function at $A^{k} \in \partial D^{k}$ is given by

$$
P_{A^{k}}(z)=\sum_{i=1}^{n} \frac{\partial \rho^{k}}{\partial z_{i}}\left(A^{k}\right)\left(z_{i}-A_{i}^{k}\right)+\frac{1}{2} \sum_{i, j=1}^{n} \frac{\partial^{2} \rho^{k}}{\partial z_{i} \partial z_{j}}\left(A^{k}\right)\left(z_{i}-A_{i}^{k}\right)\left(z_{j}-A_{j}^{k}\right) .
$$

Since $\rho^{k}$ converges to $\rho^{0}$ in the $C^{2}$-topology, the neighbourhoods $D_{1}^{k}$ can be chosen uniformly and the computations in 13 remain valid.

The following simple consequence of the Schwarz lemma on the unit disc will be needed:

Lemma 9.4. For each $\lambda>0$ there exists a positive constant $C_{\lambda}$ such that for every $\epsilon>0$ and every holomorphic disc $g: \Delta \rightarrow \Delta$ satisfying $|1-g(0)| \leq \epsilon$ we have

$$
|1-g(\zeta)| \leq \lambda
$$

whenever $|\zeta| \leq 1-C_{\lambda} \epsilon$.

Using this it is possible to control the behaviour of analytic discs in $D_{1}^{k}$ near $A^{k}$.

Lemma 9.5. Let $D, D^{k}$ and $D_{1}^{k}$ be as described before. For $\eta>0$, there exist uniform positive constants $C$ and $\delta$ such that for every holomorphic disc $h: \Delta \rightarrow D_{1}^{k}$ satisfying $d\left(h^{k}(0), \partial D^{k}\right)<\delta$,

$$
\left|h^{k}(z)-h^{k}(0)\right|<\eta
$$

whenever $|z| \leq 1-C d\left(h^{k}(0), \partial D^{k}\right)$.

Proof. For every holomorphic mapping $h^{k}: \Delta \rightarrow D_{1}^{k}$, choose points $B^{k}$ on $\partial D^{k} \cap \partial D_{1}^{k}$ closest to $h^{k}(0)$. It follows from lemma 9.3 there exist uniform positive constants $C_{1}$ and $C_{2}$ such that the local peak function at $B^{k}$ satisfies

$$
\left|1-P_{B^{k}}\left(h^{k}(0)\right)\right| \leq\left|h^{k}(0)-B^{k}\right| / C_{1},\left|h^{k}(z)-B^{k}\right| \leq C_{2} \sqrt{\left|1-P_{B^{k}}\left(h^{k}(z)\right)\right|}
$$

for all $z \in \Delta$. Now, applying lemma 9.4 to the holomorphic discs $P_{B^{k}} \circ h^{k}: \Delta \rightarrow \Delta$ for $\lambda=\left(\eta / 2 C_{2}\right)^{2}$ yields

$$
\left|1-P_{B^{k}}\left(h^{k}(z)\right)\right| \leq \lambda
$$


whenever $|z| \leq 1-C_{\lambda} / C_{1}\left|h^{k}(0)-B^{k}\right|$. Thus, for all such $z$

$$
\begin{aligned}
\left|h^{k}(z)-h^{k}(0)\right| & \leq\left|h^{k}(z)-B^{k}\right|+\left|B^{k}-h^{k}(0)\right| \\
& \leq C_{2} \sqrt{\left|1-P_{B^{k}}\left(h^{k}(z)\right)\right|}+\left|h^{k}(0)-B^{k}\right| \\
& \leq C_{2} \sqrt{\lambda}+\left|h^{k}(0)-B^{k}\right|<\eta
\end{aligned}
$$

provided $\left|h^{k}(0)-B^{k}\right|<\eta / 2$ for all $k$. Choosing $\delta=\eta / 2$ and $C=C_{\lambda} / C_{1}$, we get the required result.

Lemma 9.6. For each fixed boundary point $A^{k}$ of $D^{k}$, there exists a uniform positive constant $C$ and a neighbourhood of $A^{k}$ of uniform size $D_{3}^{k}$ which is compactly contained in $D_{1}^{k}$ such that

$$
F_{D^{k}}^{K}(z, X) \geq\left(1-C d\left(z, \partial D^{k}\right)\right) F_{D_{1}^{k}}^{K}(z, X)
$$

for every $z$ in $D_{3}^{k}$ and $X$ a tangent vector at $z$.

Proof. Since $D$ is bounded, we may assume that the diameter of $D^{k}$ is at most one for all $k$. Fix $A^{k} \in \partial D^{k}$. Choose $d \in(0,1)$ and a neighbourhood $D_{2}^{k}$ of $A^{k}$ of uniform size which is relatively compact in $D_{1}^{k}$ such that

$$
B(z, d) \cap D^{k} \subset D_{1}^{k}
$$

whenever $z \in D_{2}^{k}$. Given $\epsilon>0$ sufficiently small, let $\alpha^{k}=\alpha^{k}(\epsilon)$ be the largest number in $[0,1]$ such that $\left|h^{k}(z)-h^{k}(0)\right| \leq d$ whenever $h^{k}: \Delta \rightarrow D^{k}$ is a holomorphic mapping with $h^{k}(0) \in D_{2}^{k}, d\left(h^{k}(0), \partial D^{k}\right) \leq \epsilon$ and $|z| \leq \alpha^{k}$. Observe that the Schwarz lemma implies that $d \leq \alpha^{k}$. Further, note that $h^{k}\left(\Delta\left(0, \alpha^{k}\right)\right) \subset$ $B\left(h^{k}(0), d\right) \cap D^{k}$ and $B\left(h^{k}(0), d\right) \cap D^{k} \subset D_{1}^{k}$ for all $k$. Now, applying lemma 9.5 to the mapping $\left.h^{k}\right|_{\Delta\left(0, \alpha^{k}\right)}$ yields uniform positive constants $\tilde{C}$ and $\delta$ such that if $d\left(h^{k}(0), \partial D^{k}\right)<\delta$ then $\left|h^{k}(z)-h^{k}(0)\right| \leq d / 2$ whenever $|z| \leq \alpha^{k}\left(1-\tilde{C} d\left(h^{k}(0), \partial D^{k}\right)\right)$. In particular,

$$
\left|h^{k}(z)-h^{k}(0)\right| \leq d / 2
$$

for $|z| \leq \alpha^{k}-\tilde{C} \epsilon$. Let

$$
d^{k}=\sup \left\{\left|h^{k}(z)-h^{k}(0)\right|:|z|=\alpha^{k}, h^{k} \in \mathcal{O}\left(\Delta\left(0, \alpha^{k}\right) ; D_{1}^{k}\right), h^{k}(0) \in D_{2}^{k} \text { and } d\left(h^{k}(0), \partial D^{k}\right)<\delta\right\} .
$$

Observe that $d^{k} \leq d$ for all $k$. Furthermore, by Hadamard's three circle lemma, the function

$$
\log \sup _{|z|=r}\left|h^{k}(z)-h^{k}(0)\right|
$$

is a convex function of $\log r$. Therefore,

which implies that

$$
\frac{\log d / 2}{\log \left(\alpha^{k}-\tilde{C} \epsilon\right)} \leq \frac{\log d^{k}}{\log \alpha^{k}} \leq \frac{\log d}{\log \alpha^{k}}
$$

$$
\left|\frac{\log \alpha^{k}}{\log d}\right| \leq\left|\frac{\log \left(\alpha^{k}-\tilde{C} \epsilon\right)}{\log d / 2}\right|
$$

Since

$$
\lim _{\epsilon \rightarrow 0}(1 / \epsilon) \log \left(1-\tilde{C} \epsilon / \alpha^{k}\right)=-\tilde{C} / \alpha^{k} \geq-\tilde{C} / d
$$

it follows that

This shows that for all $k$

$$
1-\alpha^{k} \leq\left|\log \alpha^{k}\right| \leq 2 \tilde{C}|\log d| \epsilon /(d \log 2) .
$$

$$
\alpha^{k} \geq 1-2 \tilde{C}|\log d| \epsilon /(d \log 2) .
$$

Set $C=2 \tilde{C}|\log d| /(d \log 2)$ and $D_{3}^{k}=B\left(A^{k}, \delta\right) \cap D_{2}^{k}$. To summarise, we have proved the following: There exists a uniform positive constant $C$ and uniform neighbourhoods $D_{3}^{k}$ compactly contained in $D_{1}^{k}$ such that for every holomorphic mapping $h^{k}: \Delta \rightarrow D^{k}$ satisfying $h^{k}(0) \in D_{3}^{k}$,

$$
h^{k}(\Delta(0,1-C \epsilon)) \subset D_{1}^{k} .
$$

The required result then follows from the definition of the infinitesimal Kobayashi metric. 
Lemma 9.7. Let $D, D^{k}, D_{1}^{k}$ and $D_{3}^{k}$ be as described before. For each $A^{k} \in \partial D^{k}$, there exist neighbourhoods $D_{4}^{k}$ compactly contained in $D_{3}^{k}$ such that for a suitable uniform constant $C^{\prime}>0$,

$$
d_{D^{k}}\left(z, D^{k} \backslash D_{1}^{k}\right) \geq-(1 / 2) \log d\left(z, \partial D^{k}\right)-C^{\prime}
$$

for all $z$ in $D_{4}^{k}$.

Proof. Fix $A^{k} \in \partial D^{k}$. Choose a sufficiently small neighbourhood $D_{4}^{k}$ of $A^{k}$ of uniform size, $D_{4}^{k}$ compactly contained in $D_{3}^{k}$ such that for each point $z \in D_{4}^{k} \cap D^{k}$, the point $A^{z} \in \partial D_{4}^{k} \cap \partial D^{k}$ closest to $z$ satisfies

$$
\lim _{z^{\prime} \in D^{k} \backslash D_{3}^{k}}\left|A^{z}-z^{\prime}\right| \geq \delta_{0}>0
$$

where $\delta_{0}$ can be chosen to be independent of the point $z$ and the index $k$. This is possible since $D_{3}^{k}$ and $D_{1}^{k}$ are of uniform size. Pick $z \in D_{4}^{k}$ and $w^{k} \in D^{k} \backslash D_{1}^{k}$. Let $\sigma^{k}$ be any arbitrary piecewise $C^{1}$-smooth curve in $D^{k}$ joining $w^{k}$ and $z$. As we travel along $\sigma^{k}$ there is a first point $\tilde{z}^{k}$ on the curve with $\tilde{z}^{k} \in \partial D_{3}^{k} \cap D^{k}$. Let $\gamma^{k}$ be the subcurve of $\sigma^{k}$ joining $\tilde{z}^{k}$ and $z$. Choose points $B^{k} \in \partial D_{4}^{k} \cap \partial D^{k}$ closest to the point $z$. It is already known that the local peak function $P_{B^{k}}$ at $B^{k}$ is holomorphic on $D_{1}^{k}$. Hence,

$$
\lambda^{k}(t)=P_{B^{k}}\left(\gamma^{k}(t)\right)
$$

is well-defined. Set $\xi^{k}(t)=\left|\lambda^{k}(t)\right|$. The explicit expression for $P_{B^{k}}$ shows that it has no zeroes inside $\overline{D_{1}^{k}}$. Hence, $\xi^{k}$ is $C^{1}$-smooth. It follows from lemma 9.6 that

$$
\int_{0}^{1} F_{D^{k}}^{K}\left(\gamma^{k}(t), \dot{\gamma}^{k}(t)\right) d t \geq \int_{0}^{1}\left(1-C d\left(\gamma^{k}(t), \partial D^{k}\right)\right) F_{D_{1}^{k}}^{K}\left(\gamma^{k}(t), \dot{\gamma}^{k}(t)\right) d t
$$

Since holomorphic maps decrease the Kobayashi metric, we have

$$
\begin{aligned}
\int_{0}^{1}\left(1-C d\left(\gamma^{k}(t), \partial D^{k}\right)\right) F_{D_{1}^{k}}^{K}\left(\gamma^{k}(t), \dot{\gamma}^{k}(t)\right) d t & \geq \int_{0}^{1}\left(1-C d\left(\gamma^{k}(t), \partial D^{k}\right)\right) F_{\Delta}^{K}\left(\lambda^{k}(t), \dot{\lambda}^{k}(t)\right) d t \\
& =\int_{0}^{1}\left(1-C d\left(\gamma^{k}(t), \partial D^{k}\right)\right) \frac{\left|\dot{\lambda}^{k}(t)\right|}{1-\left|\lambda^{k}(t)\right|^{2}} d t \\
& =\int_{0}^{1}\left(1-C d\left(\gamma^{k}(t), \partial D^{k}\right)\right) \frac{\dot{\xi}^{k}(t)}{1-\xi^{k}(t)^{2}} d t \\
& \geq \int_{0}^{1}\left(1-C d\left(\gamma^{k}(t), \partial D^{k}\right)\right) \frac{\dot{\xi}^{k}(t)}{2\left(1-\xi^{k}(t)\right)} d t
\end{aligned}
$$

Also, by lemma 9.3 , for every $t \in[0,1]$

$$
\begin{aligned}
1-C d\left(\gamma^{k}(t), \partial D^{k}\right) & \geq 1-C\left|\gamma^{k}(t)-B^{k}\right| \\
& \geq 1-C C_{2} \sqrt{1-\left|P_{B^{k}}\left(\gamma^{k}(t)\right)\right|} \\
& =1-C C_{2} \sqrt{1-\xi^{k}(t)}
\end{aligned}
$$

Therefore,

$$
\begin{aligned}
\int_{0}^{1} F_{D^{k}}^{K}\left(\gamma^{k}(t), \dot{\gamma}^{k}(t)\right) d t & \geq \int_{0}^{1}\left(1-C C_{2} \sqrt{1-\xi^{k}(t)}\right) \frac{\dot{\xi}^{k}(t)}{2\left(1-\xi^{k}(t)\right)} d t \\
& \geq-(1 / 2) \log \left(1-\xi^{k}(1)\right)+(1 / 2) \log \left(1-\xi^{k}(0)\right)+\left(C C_{2} / 2\right) \int_{0}^{1} \frac{d x}{\sqrt{x}}
\end{aligned}
$$

Observe that

$$
\begin{aligned}
& 1-\xi^{k}(1)=1-\left|P_{B^{k}}\left(\gamma^{k}(1)\right)\right| \leq\left|\gamma^{k}(1)-B^{k}\right| / C_{1}=d\left(\gamma^{k}(1), \partial D^{k}\right) / C_{1} \\
& 1-\xi^{k}(0)=1-\left|P_{B^{k}}\left(\gamma^{k}(0)\right)\right| \geq\left|\gamma^{k}(0)-B^{k}\right|^{2} \geq \delta_{0}{ }^{2}
\end{aligned}
$$

This implies that

$$
\begin{aligned}
\int_{0}^{1} F_{D^{k}}^{K}\left(\gamma^{k}(t), \dot{\gamma}^{k}(t)\right) d t & \geq \frac{1}{2} \log \left(\frac{C_{1}}{d\left(\gamma^{k}(1), \partial D^{k}\right)}\right)-\frac{1}{2} \log \left(\frac{1}{\delta_{0}{ }^{2}}\right)+\frac{C C_{2}}{2} \int_{0}^{1} \frac{d x}{\sqrt{x}} \\
& \geq-(1 / 2) \log d\left(z, \partial D^{k}\right)-C^{\prime}
\end{aligned}
$$


where

This shows that

$$
C^{\prime}=(1 / 2) \log C_{1}+\log \left(\delta_{0}\right)+\left(C C_{2} / 2\right) \int_{0}^{1} \frac{d x}{\sqrt{x}} .
$$

$$
\int_{0}^{1} F_{D^{k}}^{K}\left(\sigma^{k}(t), \dot{\sigma}^{k}(t)\right) d t \geq-(1 / 2) \log d\left(z, \partial D^{k}\right)-C^{\prime} .
$$

Taking the infimum over all admissible curves, we get the required result.

Proof of Proposition 9.1; For each $k \geq 1$ choose points $X^{k}$ and $Y^{k}$ on $\partial D^{k}$ closest to $a$ and $b$ respectively. Each path in $D^{k}$ joining $a$ and $b$ must exit from neighbourhoods of $X^{k}$ and $Y^{k}$. Hence the result is immediate from lemma 9.7 .

Lemma 9.8. Let $D$ and $D^{k}$ be as described before. There exists a uniform positive constant $C$ such that

$$
d_{D^{k}}\left(x, x^{k}\right) \leq \frac{1}{2} \log \left(\frac{d\left(x^{k}, \partial D^{k}\right)}{d\left(x, \partial D^{k}\right)}\right)+C
$$

where $x \in D$ is sufficiently close to $\partial D$ and the points $x^{k} \in D^{k}$ lie on the same normal to $D^{k}$ as $x$.

Proof. This follows by integrating the infinitesimal metric along the straight line path in $D^{k}$ joining $x$ and $x^{k}$.

Proof of Proposition 9.2: For each $k$, we denote by $n^{k}(q)$ the unit outward normal to $\partial D^{k}$ at $q \in \partial D^{k}$. Fix $C^{k} \in \partial D^{k}$ temporarily. Since $\rho^{k}$ converges to $\rho^{0}$ in $C^{2}$-topology, there exists a $0<R \ll 1$ such that

(i) $\left|n^{k}(q)-n^{k}\left(C^{k}\right)\right|<1 / 8$ for $q \in \partial D^{k} \cap B\left(C^{k}, R\right)$,

(ii) $z-\delta n^{k}(q) \in D^{k}$ and $d\left(z-\delta n^{k}(q), \partial D^{k}\right)>3 \delta / 4$ whenever $z \in D^{k} \cap B\left(C^{k}, R\right), q \in \partial D^{k} \cap B\left(C^{k}, 8 R\right)$ and $\delta \leq 2 R$.

Note that $R$ is independent of $C^{k}$ and $k$ for $k$ large. It is already known that there exist uniform positive constants $C_{1}$ and $C_{2}$ such that for every $z$ in $N_{\epsilon}(\partial D)$

$$
C_{1} d(z, \partial D) \leq d\left(z, \partial D^{k}\right) \leq C_{2} d(z, \partial D)
$$

for all $k$ large. Now, choose $r \in\left(0, R / 4 C_{2}\right)$ and fix two points $a, b \in D \cap B\left(q^{0}, r\right)$. Let $A^{k}, B^{k} \in \partial D^{k}$ be the uniquely determined points closest to $a$ and $b$ respectively. Next, find $\tilde{C}^{k} \in \partial D^{k}$ such that $a, b \in B\left(\tilde{C}^{k}, R\right)$ for all $k$ large. Setting

$$
a^{k}=a-|a-b| n^{k}\left(A^{k}\right) \text { and } b^{k}=b-|a-b| n^{k}\left(B^{k}\right),
$$

note that

$$
\left|A^{k}-\tilde{C}^{k}\right| \leq\left|A^{k}-a\right|+\left|a-\tilde{C}^{k}\right|<R / 4+R<2 R .
$$

Similarly, $B^{k} \in B\left(\tilde{C}^{k}, 2 R\right)$. It follows from (ii) that $a^{k}, b^{k} \in D^{k}, d\left(a^{k}, \partial D^{k}\right)>(3 / 4)|a-b|$ and $d\left(b^{k}, \partial D^{k}\right)>(3 / 4)|a-b|$. The triangle inequality gives the following upper estimate:

$$
d_{D^{k}}(a, b) \leq d_{D^{k}}\left(a, a^{k}\right)+d_{D^{k}}\left(a^{k}, b^{k}\right)+d_{D^{k}}\left(b^{k}, b\right)
$$

By lemma 9.8, we conclude that there exists a uniform positive constant $C$ such that

$$
\begin{gathered}
d_{D^{k}}\left(a, a^{k}\right) \leq(1 / 2) \log \left(\frac{d\left(a^{k}, \partial D^{k}\right)}{d\left(a, \partial D^{k}\right)}\right)+C \\
d_{D^{k}}\left(b, b^{k}\right) \leq(1 / 2) \log \left(\frac{d\left(b^{k}, \partial D^{k}\right)}{d\left(b, \partial D^{k}\right)}\right)+C
\end{gathered}
$$

Also, by construction we have

$$
d\left(a^{k}, \partial D^{k}\right)=d\left(a, \partial D^{k}\right)+|a-b|, d\left(b^{k}, \partial D^{k}\right)=d\left(b, \partial D^{k}\right)+|a-b| .
$$

It remains to estimate the term $d_{D^{k}}\left(a^{k}, b^{k}\right)$. First, it follows from (i) that $\left|a^{k}-b^{k}\right| \leq(5 / 4)|a-b|$. Now, consider the analytic discs $\phi^{k}: \mathbf{C} \rightarrow \mathbf{C}^{n}$ defined by $\phi^{k}(\lambda)=a^{k}+\lambda\left(b^{k}-a^{k}\right)$. If $|\lambda|<3 / 5$ (respectively $|\lambda-1|<3 / 5)$, we obtain

$$
\begin{aligned}
\left|\phi^{k}(\lambda)-a^{k}\right| & <(3 / 5)(5 / 4)|a-b|=(3 / 4)|a-b|<d\left(a^{k}, \partial D^{k}\right) \\
\text { (respectively }\left|\phi^{k}(\lambda)-b^{k}\right| & \left.<(3 / 5)(5 / 4)|a-b|=(3 / 4)|a-b|<d\left(b^{k}, \partial D^{k}\right)\right)
\end{aligned}
$$


i.e., if $\Omega=\Delta(0,3 / 5) \cup \Delta(1,3 / 5)$, then each $\phi^{k}$ is a holomorphic mapping of $\Omega$ into $D^{k}$. Since holomorphic maps decrease the Kobayashi metric, it follows that for all $k$

$$
d_{D^{k}}\left(a^{k}, b^{k}\right) \leq d_{\Omega}(0,1) .
$$

This completes the proof of the proposition.

Theorem 9.9. Let $D_{1}, D_{2}$ be two bounded strongly pseudoconvex domains in $\mathbf{C}^{n}$ with $C^{2}$-smooth boundaries. Let $D_{1}^{k}, D_{2}^{k}$ for $k \geq 1$ be two sequences of domains that converge to $D_{1}, D_{2}$ respectively in the $C^{2}$ topology. Suppose that $\bar{f}^{k}:\left(D_{1}^{k}, d_{D_{1}^{k}}\right) \rightarrow\left(D_{2}^{k}, d_{D_{2}^{k}}\right)$ is a $C^{0}$-smooth isometry for each $k \geq 1$ and that there is a point $p^{1} \in D_{1}$ such that some subsequence $\left\{f^{k_{j}}\left(p^{1}\right)\right\}$ converges to a point $p^{2} \in D_{2}$. Then the sequence $\left\{f^{k}\right\}$ admits a subsequence that converges uniformly on compact sets of $D_{1}$ to a continuous mapping $f: D_{1} \rightarrow D_{2}$. Moreover, $f:\left(D_{1}, d_{D_{1}}\right) \rightarrow\left(D_{2}, d_{D_{2}}\right)$ is a $C^{0}$-isometry. Further, assuming that each $f^{k} \in C^{1}\left(D_{1}^{k}\right)$, there is a uniform constant $C>0$ with the property that:

$$
\left|f^{k_{j}}(p)-f^{k_{j}}(q)\right| \leq C|p-q|^{1 / 2}
$$

for all $p, q \in D_{1}$.

Our purpose is to prove that the family $\left\{f^{k_{j}}\right\}$ is 'uniformly Hölderian' up to the boundary, i.e., every mapping extends as a Hölder continuous one up to the boundary with the Hölder constant independent of $k$. In particular, this implies the normality of this family on $\bar{D}_{1}$.

Proof. The proof involves several steps.

Step I: Let $\left\{K_{\nu}\right\}_{\nu=1}^{\infty}$ be an increasing sequence of relatively compact subsets of $D_{1}$ that exhausts $D_{1}$. Fix a pair $K_{\nu_{0}}$ compactly contained in $K_{\nu_{0}+1}$ such that $p^{1} \in K_{1}$ and write $K_{1}=K_{\nu_{0}}$ and $K_{2}=K_{\nu_{0}+1}$ for brevity. Let $\omega\left(K_{1}\right)$ be a neighbourhood of $K_{1}$ such that $\omega\left(K_{1}\right) \subset K_{2}$. Since $\left\{D_{1}^{k}\right\}$ converges to $D_{1}$, it follows that $K_{1} \subset \omega\left(K_{1}\right) \subset K_{2}$ which in turn is relatively compact in $D_{1}^{k}$ for all $k$ large. We show that the sequence $\left\{f^{k}\right\}$ is equicontinuous at each point of $\omega\left(K_{1}\right)$.

For each $x^{1} \in \omega\left(K_{1}\right)$ fixed, there exists a $r>0$ such that $B\left(x^{1}, r\right)$ is compactly contained in $\omega\left(K_{1}\right)$. The distance decreasing property of the Kobayashi metric together with its explicit form on $B\left(x^{1}, r\right)$ gives

$$
d_{D_{2}^{k}}\left(f^{k}\left(x^{1}\right), f^{k}\left(y^{1}\right)\right)=d_{D_{1}^{k}}\left(x^{1}, y^{1}\right) \leq d_{B\left(x^{1}, r\right)}\left(x^{1}, y^{1}\right) \leq\left|x^{1}-y^{1}\right| / c
$$

for all $k$ large, $y^{1} \in B\left(x^{1}, r\right)$ and a uniform constant $c>0$. Since $D_{2}$ is bounded, $D_{2}^{k}$ are compactly contained in $B(0, R)$ for some $R>0$ and for all $k$ large . Consequently,

$$
d_{B(0, R)}\left(f^{k}\left(x^{1}\right), f^{k}\left(y^{1}\right)\right) \leq d_{D_{2}^{k}}\left(f^{k}\left(x^{1}\right), f^{k}\left(y^{1}\right)\right)
$$

for all $k$ large. Again using the explicit form of the metric on $B(0, R)$ gives

$$
\left|f^{k}\left(x^{1}\right)-f^{k}\left(y^{1}\right)\right| \lesssim\left|x^{1}-y^{1}\right|
$$

for $y^{1} \in B\left(x^{1}, r\right)$. This shows that $\left\{f^{k}\right\}$ is equicontinuous at each point if $\omega\left(K_{1}\right)$. The diagonal subsequence still denoted by the same symbols then converges uniformly on compact subsets of $D_{1}$ to a limit mapping $f: D_{1} \rightarrow \bar{D}_{2}$ which is continuous.

Step II: The proof of lemma 5.2 shows that for $i=1,2$,

$$
F_{D_{i}^{k}}^{K}(\cdot, \cdot) \rightarrow F_{D_{i}}^{K}(\cdot, \cdot)
$$

uniformly on compact sets of $D_{i} \times \mathbf{C}^{n}$ as $k \rightarrow \infty$.

Step III: Consequently,

$$
d_{D_{i}^{k}}(\cdot, \cdot) \rightarrow d_{D_{i}}(\cdot, \cdot)
$$

as $k \rightarrow \infty$.

To show this, for $p, q \in D_{1}$, firstly note that

$$
\limsup _{k \rightarrow \infty} d_{D_{1}^{k}}(p, q) \leq d_{D_{1}}(p, q)
$$

which follows exactly as in lemma 4.3 For the converse, pick $R \gg d_{D_{1}}(p, q)$ and consider $B_{D_{1}^{k}}(p, R)$. Note that $q \in B_{D_{1}^{k}}(p, R)$ and $B_{D_{1}^{k}}(p, R)$ is compactly contained in $D_{1}^{k}$ for all $k$ large. We claim that 
$B_{D_{1}^{k}}(p, R) \subset D_{1}$ for all $k$ large. Let $w^{k} \in B_{D_{1}^{k}}(p, R)$. Observe that the only case to be investigated is when $w^{k}$ is very close to the boundary of $D_{1}^{k}$. In that case, it follows from lemma 9.7 that

$$
R \gg d_{D_{1}}(p, q)+\epsilon \geq d_{D_{1}^{k}}(p, q) \geq-(1 / 2) \log d\left(w^{k}, \partial D_{1}^{k}\right)-C
$$

for some uniform positive constant $C$ and $\epsilon>0$ given. As a result,

$$
d\left(w^{k}, \partial D_{1}^{k}\right)>1 / e^{2(C+R)}>0
$$

Let $w^{k}$ converge to some point $w \in N_{\epsilon}\left(\partial D_{1}\right)$. In fact, since $D_{1}^{k}$ converge to $D_{1}$ in the $C^{2}$-topology, $w \in \bar{D}_{1}$. Further, it follows from (9.4) that $d\left(w, \partial D_{1}\right)>0$ and consequently that $w^{k} \in D_{1}$ for all $k$ large. Since the points $w^{k}$ were arbitrarily chosen, this completes the proof of the claim. Now, it follows from the distance-decreasing property of the Kobayashi metric that

$$
d_{D_{1}}(p, q) \leq d_{B_{D_{1}^{k}}(p, R)}(p, q)
$$

Applying lemma 5.4 to the domain $D_{1}^{k}$ with $B_{D_{1}^{k}}(p, R)$ as the subdomain $D^{\prime}$ yields

$$
d_{B_{D_{1}^{k}}(p, R)}(p, q) \leq \frac{d_{D_{1}^{k}}(p, q)}{\tanh \left(R / 2-d_{D_{1}^{k}}(p, q)\right)} .
$$

Using (9.3), we have

Letting $R \rightarrow \infty$ yields

$$
d_{D_{1}}(p, q) \leq \frac{d_{D_{1}^{k}}(p, q)}{\tanh \left(R / 2-d_{D_{1}}(p, q)-\epsilon\right)}
$$

$$
d_{D_{1}}(p, q) \leq d_{D_{1}^{k}}(p, q)+\epsilon
$$

for all $k$ large. Putting together (9.3) and (9.5), we get

$$
\lim _{k \rightarrow \infty} d_{D_{1}^{k}}(p, q)=d_{D_{1}}(p, q) .
$$

The same proof works for the domain $D_{2}$ and is therefore omitted.

Step IV: We now show that $d_{D_{1}}\left(x^{1}, y^{1}\right)=d_{D_{2}}\left(f\left(x^{1}\right), f\left(y^{1}\right)\right)$ for all $x^{1}, y^{1} \in \Omega_{1}$ where $\Omega_{1}=\left\{q^{1} \in D_{1}\right.$ : $\left.f\left(q^{1}\right) \in D_{2}\right\}$. Note that $\Omega_{1}$ is non-empty since $p^{1} \in D_{1}$. Let $x^{1}, y^{1} \in \Omega_{1}$. It is known that

$$
d_{D_{1}^{k}}\left(x^{1}, y^{1}\right)=d_{D_{2}^{k}}\left(f^{k}\left(x^{1}\right), f^{k}\left(y^{1}\right)\right) .
$$

for all $k$ and thus by (9.2), it remains to show that the right side above converges to $d_{D_{2}}\left(f\left(x^{1}\right), f\left(y^{1}\right)\right)$ as $k \rightarrow \infty$. For this note that

$$
\left|d_{D_{2}^{k}}\left(f^{k}\left(x^{1}\right), f^{k}\left(y^{1}\right)\right)-d_{D_{2}^{k}}\left(f\left(x^{1}\right), f\left(y^{1}\right)\right)\right| \leq d_{D_{2}^{k}}\left(f^{k}\left(x^{1}\right), f\left(x^{1}\right)\right)+d_{D_{2}^{k}}\left(f\left(y^{1}\right), f^{k}\left(y^{1}\right)\right)
$$

by the triangle inequality. Since $f^{k}\left(x^{1}\right) \rightarrow f\left(x^{1}\right)$ and the domains $D_{2}^{k}$ converge to $D_{2}$, it follows that there is a small ball $B\left(f\left(x^{1}\right), r\right)$ around $f\left(x^{1}\right)$ which contains $f^{k}\left(x^{1}\right)$ for all large $k$ and which is contained in $D_{2}^{k}$ for all large $k$, where $r>0$ is independent of $k$. Thus

$$
d_{D_{2}^{k}}\left(f^{k}\left(x^{1}\right), f\left(x^{1}\right)\right) \leq C\left|f^{k}\left(x^{1}\right)-f\left(x^{1}\right)\right|
$$

for some uniform constant $C$. The same argument works for showing that $d_{D_{2}^{k}}\left(f\left(y^{1}\right), f^{k}\left(y^{1}\right)\right)$ is small. So to verify the claim, it is enough to prove that $d_{D_{2}^{k}}\left(f\left(x^{1}\right), f\left(y^{1}\right)\right)$ converges to $d_{D_{2}}\left(f\left(x^{1}\right), f\left(y^{1}\right)\right)$. But this is immediate from (9.2).

Step V: The limit map $f$ is a surjection onto $D_{2}$. Firstly, we need to show that $f\left(D_{1}\right) \subset D_{2}$. Indeed, $\Omega_{1}=D_{1}$. If $q^{0} \in \partial \Omega_{1} \cap D_{1}$, choose a sequence $q^{j} \in \Omega_{1}$ that converges to $q^{0}$. It follows from Step IV that

$$
d_{D_{1}}\left(q^{j}, p^{1}\right)=d_{D_{2}}\left(f\left(q^{j}\right), f\left(p^{1}\right)\right)
$$

for all $j$. Since $q^{0} \in \partial \Omega_{1}$, the sequence $\left\{f\left(q^{j}\right)\right\}$ converges to a point on $\partial D_{2}$ and as $D_{2}$ is complete in the Kobayashi distance, the right hand side above becomes unbounded. However, the left hand side remains bounded again because of completeness of $D_{1}$. This contradiction shows that $\Omega_{1}=D_{1}$ which exactly means that $f\left(D_{1}\right) \subset D_{2}$. The above observation coupled with Step IV forces that

$$
d_{D_{1}}\left(x^{1}, y^{1}\right)=d_{D_{2}}\left(f\left(x^{1}\right), f\left(y^{1}\right)\right)
$$


for all $x^{1}, y^{1} \in D_{1}$. To establish the surjectivity of $f$, consider any point $q^{0} \in \partial\left(f\left(D_{1}\right)\right) \cap D_{2}$ and choose a sequence $q^{j} \in f\left(D_{1}\right)$ that converges to $q^{0}$. Let $\left\{p^{j}\right\}$ be sequence of points in $D_{1}$ be such that $f\left(p^{j}\right)=q^{j}$. Then for all $j$ and for all $x^{1} \in D_{1}$,

$$
d_{D_{1}}\left(x^{1}, p^{j}\right)=d_{D_{2}}\left(f\left(x^{1}\right), f\left(p^{j}\right)\right)
$$

There are two cases to be considered. After passing to a subsequence, if needed,

(i) $p^{j} \rightarrow x^{0} \in \partial D_{1}$,

(ii) $p^{j} \rightarrow x^{2} \in D_{1}$ as $j \rightarrow \infty$.

In case (i), observe that the right hand side remains bounded because of the completeness of $D_{2}$. Moreover, since $D_{1}$ is complete in the Kobayashi metric, the left hand side in (9.6) becomes unbounded. This contradiction shows that $f\left(D_{1}\right)=D_{2}$.

For (ii), firstly, the continuity of the mapping $f$ implies that the sequence $\left\{f\left(p^{j}\right)\right\}$ converges to the point $f\left(x^{2}\right)$. Therefore, we must have $f\left(x^{2}\right)=q^{0}$. Consider the mappings $\left(f^{k}\right)^{-1}: D_{2}^{k} \rightarrow D_{1}^{k}$. Arguing as before, we infer that the sequence $\left\{\left(f^{k}\right)^{-1}\right\}$ admits a subsequence that converges uniformly on compact sets of $D_{2}$ to a continuous mapping $g: D_{2} \rightarrow \bar{D}_{1}$. Then $g \circ f \equiv i d_{D_{1}}$. Therefore,

$$
x^{2}=g \circ f\left(x^{2}\right)=g\left(q^{0}\right)=\lim _{k \rightarrow \infty}\left(f^{k}\right)^{-1}\left(q^{0}\right) .
$$

Hence the sequence $\left\{\left(f^{k}\right)^{-1}\left(q^{0}\right)\right\}$ is compactly contained in $D_{1}$. Now, repeating the earlier argument for $\left\{\left(f^{k}\right)^{-1}\right\}$, it follows that $g: D_{2} \rightarrow D_{1}$ and $f \circ g \equiv i d_{D_{2}}$. In particular, $f$ is surjective.

This also shows that the limit mapping $f$ is a $C^{0}$-isometry. We note that so far we did not need the isometries to be $C^{1}$-smooth. Now, assume that each $f^{k} \in C^{1}\left(D_{1}^{k}\right)$.

The following assertion gives a 'uniform local hyperbolicity' of the family $\left\{D_{2}^{k}\right\}$ near $\partial D_{2}$.

Step VI: There exists an $\epsilon>0$ and a uniform positive constant $C$ such that for any $k \geq 1$ and $q^{2} \in D_{2}^{k} \cap N_{\epsilon}\left(\partial D_{2}\right)$ and $v \in \mathbf{C}^{n}$ we have

$$
F_{D_{2}^{k}}^{K}\left(q^{2}, v\right) \geq C \frac{|v|}{\sqrt{d\left(q^{2}, \partial D_{2}^{k}\right)}} .
$$

Fix a point $q^{2}$ near $\partial D_{2}$ and let $v \in \mathbf{C}^{n} \backslash\{0\}$. Let $R^{k}$ be a sequence of positive real numbers and $\psi^{k}$ a sequence of holomorphic mappings, $\psi^{k} \in \mathcal{O}\left(\Delta ; D_{2}^{k}\right) \cap C^{0}\left(\bar{\Delta} ; \mathbf{C}^{n}\right)$ satisfying $\psi^{k}(0)=q^{2}$ and $\left(\psi^{k}\right)^{\prime}(0)=$ $R_{k} v$. Choose $\zeta^{k} \in \partial D_{2}^{k}$ with $\left|q^{2}-\zeta^{k}\right|=d\left(q^{2}, \partial D_{2}^{k}\right)$ and let $\rho_{2}^{k}$ be a $C^{2}$ - smooth defining function for $D_{2}^{k}$ which is strictly plurisubharmonic in a neighbourhood of $\overline{D_{2}^{k}}$. Then, for $\eta>0$ sufficiently small,

$$
\tilde{\rho}_{2}^{k}(z)=\rho_{2}^{k}(z)-\eta\left|z-\zeta^{k}\right|^{2}
$$

is a strictly plurisubharmonic on a neighbourhood of closure of $D_{2}^{k}$. Therefore,

$$
\tilde{\rho}_{2}^{k}\left(q^{2}\right)=\tilde{\rho}_{2}^{k} \circ \psi^{k}(0) \leq \frac{1}{2 \pi} \int_{0}^{2 \pi} \rho_{2}^{k}\left(\psi^{k}\left(e^{\iota t}\right)\right)-\eta\left|\psi^{k}\left(e^{\iota t}\right)-\zeta^{k}\right|^{2} d t \leq-\frac{\eta}{2 \pi} \int_{0}^{2 \pi}\left|\psi^{k}\left(e^{\iota t}\right)-\zeta^{k}\right|^{2} d t .
$$

Applying the Cauchy integral formula it follows that

$$
\left|\left(\psi^{k}\right)^{\prime}(0)\right|^{2}=\sum_{j=1}^{n}\left|\frac{1}{2 \pi \iota} \int_{0}^{2 \pi} \frac{\psi_{j}^{k}\left(e^{\iota t}\right)-\zeta_{j}^{k}}{e^{2 \iota t}} d t\right|^{2} \leq \frac{1}{2 \pi} \int_{0}^{2 \pi}\left|\psi^{k}\left(e^{\iota t}\right)-\zeta^{k}\right|^{2} d t .
$$

As a consequence,

$$
\left|\left(\psi^{k}\right)^{\prime}(0)\right|^{2} \leq\left|q-\zeta^{k}\right|^{2}-\rho_{2}^{k}\left(q^{2}\right) / \eta \text {. }
$$

Now, since $\rho_{2}^{k} \rightarrow \rho_{2}^{0}$ in the $C^{2}$-topology, it follows that there exists a uniform constant $M>0$ such that

$$
\left|\rho_{2}^{k}(z)\right| \leq M d\left(z, \partial D_{2}^{k}\right)
$$

for all $k$ large and for all $z$ sufficiently close to $\partial D_{2}^{k}$. Then

$$
\left|\left(\psi^{k}\right)^{\prime}(0)\right|^{2} \leq(1+M / \eta) d\left(q^{2}, \partial D_{2}^{k}\right) .
$$

The required result then follows from the definition of the infinitesimal metric. 
Step VII: There exist uniform positive constants $A$ and $B$ such that

$$
A d\left(x, \partial D_{1}^{k}\right) \leq d\left(f^{k}(x), \partial D_{2}^{k}\right) \leq B d\left(x, \partial D_{1}^{k}\right)
$$

for all $x$ sufficiently close to $\partial D_{1}$.

To see this, fix $y \in D_{1}$ sufficiently close to $\partial D_{1}$ and $x^{0} \in \partial D_{1}$. Let $\left\{x^{j}\right\}$ be a sequence of points in $D_{1}$ converging to $x^{0}$. Then for each $j$ large there is an index $k_{0}$ such that $x^{j} \in D_{1}^{k}$ for $k \geq k_{0}$. For such a fixed large $j$, apply lemma 9.7 and lemma 9.8 to get uniform positive constants $C_{1}, C_{2}$ such that

$$
-(1 / 2) \log d\left(x^{j}, \partial D_{1}^{k}\right)-C_{2} \leq d_{D_{1}^{k}}\left(x^{j}, y\right) \leq-(1 / 2) \log d\left(x^{j}, \partial D_{1}^{k}\right)+C_{1}
$$

for all $k \geq k_{0}$. Note that $\left\{f^{k}(y)\right\}$ is compactly contained in $D_{2}$ from Step V. From [35] it follows that each $f^{k}$ is continuous up to $\overline{D_{1}^{k}}$ and $f^{k}\left(\partial D_{1}^{k}\right) \subset \partial D_{2}^{k}$. Since $D_{2}^{k}$ converges to $D_{2}$, it follows that $\left\{x^{j}\right\} \subset N_{\epsilon}\left(\partial D_{1}^{k}\right)$ for all $k$ large. Again using lemma 9.7 and lemma 9.8 yields

$$
\begin{aligned}
-(1 / 2) \log d\left(f^{k}\left(x^{j}\right), \partial D_{2}^{k}\right)-C_{3} & \leq d_{D_{2}^{k}}\left(f^{k}(y), f^{k}\left(x^{j}\right)\right) \\
& \leq-(1 / 2) \log \left(d\left(f^{k}\left(x^{j}\right), \partial D_{2}^{k}\right) d\left(f^{k}(y), \partial D_{2}^{k}\right)\right)+C_{4}
\end{aligned}
$$

for $k \geq k_{0}$ and $C_{3}, C_{4}>0$ uniform in $k$. Since $d_{D_{1}^{k}}\left(y, x^{j}\right)=d_{D_{2}^{k}}\left(f^{k}(y), f^{k}\left(x^{j}\right)\right)$, it follows from (9.7) and (9.8) that

$$
A d\left(x, \partial D_{1}^{k}\right) \leq d\left(f^{k}(x), \partial D_{2}^{k}\right) \leq B d\left(x, \partial D_{1}^{k}\right)
$$

for some uniform $A, B>0$ and $x$ sufficiently close to $\partial D_{1}$.

Step VIII: For $\zeta^{0} \in \partial D_{1}$ fixed, there exists a neighbourhood $V$ of $\zeta^{0}$ such that for all $p, q \in V \cap D_{1}$ and for all $k$ large

$$
\left|f^{k}(p)-f^{k}(q)\right| \leq C|p-q|^{1 / 2}
$$

where $C$ is a constant independent of $p, q \in V \cap D_{1}$.

Firstly, observe that given $\eta>0$ there exist $\eta^{\prime}, \eta^{\prime \prime}>0$ such that

- $f\left(\left\{z \in D_{1}: d\left(z, \partial D_{1}\right)<\eta\right\}\right) \subset\left\{w \in D_{2}: d\left(w, \partial D_{2}\right)<\eta^{\prime}\right\}$

- $f^{-1}\left(\left\{w \in D_{2}: d\left(w, \partial D_{2}\right)<\eta\right\}\right) \subset\left\{z \in D_{1}: d\left(z, \partial D_{1}\right)<\eta^{\prime \prime}\right\}$

- $\lim _{\eta \rightarrow 0} \eta^{\prime}=0$ and $\lim _{\eta \rightarrow 0} \eta^{\prime \prime}=0$.

These are consequences of the completeness of the Kobayashi distance on strongly pseudoconvex domains. Now, from Step VI there exists a uniform positive constant $C$ such that

$$
F_{D_{2}^{k}}^{K}\left(f^{k}(x), d f^{k}(x) v\right) \geq C \frac{\left|d f^{k}(x) v\right|}{\sqrt{d\left(f^{k}(x), \partial D_{2}^{k}\right)}}
$$

for all $k$ large and for all $x$ sufficiently close to $\partial D_{1}$. Using the fact that each $f^{k}$ is an isometry we get

$$
C \frac{\left|d f^{k}(x) v\right|}{\sqrt{d\left(f^{k}(x), \partial D_{2}^{k}\right)}} \leq F_{D_{2}^{k}}^{K}\left(f^{k}(x), d f^{k}(x) v\right)=F_{D_{1}^{k}}^{K}(x, v) \leq \frac{|v|}{d\left(x, \partial D_{1}^{k}\right)}
$$

for all tangent vectors $v$ at $x$. This implies that

$$
\left|d f^{k}(x) v\right| \leq C \frac{|v| \sqrt{d\left(f^{k}(x), \partial D_{2}^{k}\right)}}{d\left(x, \partial D_{1}^{k}\right)}
$$

By Step VII, we have

$$
\left|d f^{k}(x) v\right| \leq C \frac{|v|}{\sqrt{d\left(x, \partial D_{1}^{k}\right)}}
$$

Next, observe that since $\partial D_{1}$ is $C^{2}$-smooth, there exists a $0<R \ll 1$ such that

$$
z-\delta n(\zeta) \in D_{1} \text { and } d\left(z-\delta n(\zeta), \partial D_{1}\right)>3 \delta / 4
$$

for all $z \in D_{1} \cap B\left(\zeta^{0}, R\right), \zeta \in \partial D_{1} \cap B\left(\zeta^{0}, 8 R\right)$ and $\delta \leq 2 R$. Here, $n(\zeta)$ denotes the unit outward normal to $\partial D_{1}$ at $\zeta$. 
Now, fix two points $p, q \in D_{1} \cap B\left(\zeta^{0}, R\right)$. Choose $p^{0}, q^{0} \in \partial D_{1}$ closest to $p$ and $q$ respectively. Set $p^{\prime}=p-|p-q| n\left(p^{0}\right)$ and $q^{\prime}=q-|p-q| n\left(q^{0}\right)$. Let $\gamma$ be the union of three segments: the first one being the straight line path joining $p$ and $p^{\prime}$ along the inward normal to $p^{0}$, the second one being a straight line path joining $p^{\prime}$ and $q^{\prime}$ and finally the third path is taken to be the straight line path joining $q^{\prime}$ and $q^{0}$ along the inward normal to the point $q^{0}$. Integrating (9.9) along this polygonal path, we get for all $k$ large

$$
\left|f^{k}(p)-f^{k}(q)\right| \leq C|p-q|^{1 / 2}
$$

uniformly for all $p, q \in D_{1} \cap B\left(\zeta^{0}, R\right)$. This completes Step VIII.

Since Step VIII together with the $C^{1}$-smoothness assumption on the isometries immediately gives the result of theorem 9.9, we may conclude.

As a corollary of theorem 9.9, we obtain:

Corollary 9.10. Let $D_{1}$ and $D_{2}$ be two bounded strongly pseudoconvex domains in $\mathbf{C}^{n}$ with $C^{2}$-smooth boundaries. Let $f^{k}:\left(D_{1}, d_{D_{1}}\right) \rightarrow\left(D_{2}, d_{D_{2}}\right)$ be a sequence of $C^{0}$-isometries between $D_{1}$ and $D_{2}$. Suppose that there exists a point $p^{1} \in D_{1}$ such that some subsequence of $\left\{f^{k}\left(p^{1}\right)\right\}$ converges to a point of $D_{2}$, then the sequence $\left\{f^{k}\right\}$ admits a subsequence $\left\{f^{k_{j}}\right\}$ that converges uniformly on compact sets of $D_{1}$ to a continuous mapping $f: D_{1} \rightarrow D_{2}$. Moreover, $f:\left(D_{1}, d_{D_{1}}\right) \rightarrow\left(D_{2}, d_{D_{2}}\right)$ is a $C^{0}$-isometry. Further, assuming that each $f^{k} \in C^{1}\left(D_{1}^{k}\right)$, there is a uniform constant $C>0$ with the property that:

$$
\left|f^{k_{j}}(p)-f^{k_{j}}(q)\right| \leq C|p-q|^{1 / 2}
$$

for all $p, q \in D_{1}$.

\section{REFERENCES}

[1] G. Aladro, The comparability of the Kobayashi approach region and the admissible approach region, Illinois J. Math., 33(1989), 42-63

[2] H. Alexander and J. Wermer, Several complex variables and Banach algebras, Third edition, Graduate Texts in Mathematics, 35, Springer-Verlag, New York, 1998.

[3] F. Berteloot, Characterisation of models in $\mathbf{C}^{2}$ by their auotmorphism groups, Internat. J. Math., 5(1994), 619-634

[4] F. Berteloot and G. Coeuré, Domaines de $\mathbf{B}^{2}$, pseudoconvexes et de type fini ayant un groupe non compact d'automorphismes, Ann. Inst. Fourier (Grenoble), 41(1991), 77-86

[5] D. Catlin, Estimates of invariant metrics on pseudoconvex domains of dimension two, Math. Z., 200(1989), 429-466

[6] B. Coupet, Uniform extendibility of automorphisms, Contemp. Math., 137(1992), 177-183

[7] B. Coupet, H. Gaussier and A. Sukhov, Regularity of CR maps between convex hypersurfaces of finite type, Proc. Amer. Math. Soc., 127(1999), 3191-3200.

[8] B. Coupet and S. I. Pinchuk, Holomorphic equivalence problem for weighted homogeneous rigid domains in $\mathbf{C}^{n+1}$, Complex analysis in modern mathematics (Russian), FAZIS, (2001), 57-70

[9] B. Coupet and A. Sukhov, On CR mappings between pseudoconvex hypersurfaces of finite type in $\mathbf{C}^{2}$, Duke Math. J., 88(1997), 381-304

[10] B. Coupet, S. Pinchuk and A. Sukhov, On boundary rigidity and regularity of holomorphic mappings, Internat. J. Math., 7(1996), 617-643

[11] B. Coupet and A. Sukhov, On the uniform extendibility of proper holomorphic mappings, Complex Variables Theory Appl., 28(1996), 243-248

[12] J. E. Fornaess and N. Sibony, Increasing sequence of complex manifolds, Math. Ann., 255(1981), 351-360

[13] F. Forstneric and J.P. Rosay, Loocalisation of the Kobayashi metric and the boundary continuity of proper holomorphic mappings, Math. Ann., 279(9187), 239-252

[14] B. L. Fridman, Biholomorphic invariants of a hyperbolic manifold and some applications, Trans. Amer. Math. Soc., 276(1983), 685-698

[15] H. Gaussier, Characterization of convex domains with noncompact automorphism group, Michigan Math. J., 44(1997), 375-388

[16] Ian Graham, Boundary behaviour of the Carathéodory and Kobayashi metrics on strongly pseudonconvex domains in $\mathbf{C}^{n}$ with smooth boundary, Trans. Amer. Math. Soc., 207(1975), 219-240

[17] R. E. Greene and S. G. Krantz, Deformation of complex structures, estimates for the $\bar{\partial}$ equation and stability of the Bergman kernel, Adv. in Math. 43(1982), no. 1, 1-86.

[18] R. E. Greene and S. G. Krantz, Stability of the Carathodory and Kobayashi metrics and applications to biholomorphic mappings, In: Complex analysis of several variables (Madison, Wis., 1982), 77-93, Proc. Sympos. Pure Math., 41, Amer. Math. Soc., Providence, RI, 1984.

[19] G. Herbort, Estimation on invariant distances on the pseudoconvex domains of finite type in dimension two, Math. Z., 251(2005), 673-703

[20] K. Hoffman, Minimal boundaries for analytic polyhedra, Rend. Circ. Mat. Palermo, 9(1960), 147-160

[21] M. Jarnicki and P. Pflug, Invariant distances and metrics in Complex Analysis, Walter de Gruyter and Co. 
[22] K.T. Kim and S. G. Krantz, A Kobayashi metric version of Bun Wong's Theorem, Complex Var. Elliptic Equ., 54(2009), 355-369

[23] K.T. Kim and D. Ma, Characterisation of the Hilbert ball by its automorphisms, J. Korean Math. Soc., 40(2003), 503-516

[24] K.T. Kim and A. Pagano, Normal analytic polyhedra in $\mathbf{C}^{2}$ with a noncompact automorphism group, J. Geom. Anal., 11(2001), 283-293

[25] S. G. Krantz, Invariant metrics and the boundary behavior of holomorphic functions on domains in $\mathbf{C}^{n}$, J. Geom. Anal., 1(1991), 71-97

[26] L. Lempert, La métrique de Kobayashi et la représentation des domaines sur la boule, Bull. Soc. Math. France, 109(1981), 427-474

[27] J. D. McNeal, Convex domains of finite type, J. Funct. Anal., 108(1992), 361-373

[28] J. D. McNeal, Estimates on the Bergman kernels of convex domains, Adv. Math., 109(1994), 108-139

[29] A. Nagel, E. M. Stein and S. B. Wainger, Balls and metrics defined by vector fields I : Basic properties, Acta Math., 155(1985), 103-147.

[30] S. I. Pinchuk, Holomorphic inequivalence of certain classes of domains in $\mathbf{C}^{n}$, Math. USSR Sb. (N.S.), 39(1980), 61-86

[31] S. I. Pinchuk, Homogeneous domains with piecewise smooth boundaries, Mat. Zametki, 32(1982), 729-735

[32] J-P. Rosay, Sur une caractèrisation de la boule parmi les domaines de $\mathbf{C}^{n}$ par son groupe d'automorphismes, Ann. Inst. Fourier (Grenoble), 29(1979), 91-97

[33] H. L. Royden, Remarks on the Kobayashi metric, Lecture Notes in math., 185(1971), 125-137

[34] Walter Rudin, Function theory in the unit ball of $\mathbf{C}^{n}$, Springer-Verlag.

[35] H. Seshadri and K. Verma, On isometries of the Carathéodory and Kobayashi metrics on strongly pseudoconvex domains, Ann. Scuola Norm. Sup. Pisa Cl. Sci.(5), V(2006), 393-417

[36] H. Seshadri and K. Verma, On the compactness of isometry groups in complex analysis, Complex Var. Elliptic Equ., 54(2009), 387-399

[37] S. Venturini, Comparison between the Kobayashi and Carathéodory distances on strongly pseudoconvex bounded domains in $\mathbf{C}^{n}$, Proc. Amer. Math. Soc., 107(1989), 725-730

[38] S. M. Webster, On the mapping problem for algebraic real hypersurfaces, Invent. Math, 43(1977), 53-68

[39] B. Wong, Characterisation of the unit ball in $\mathbf{C}^{n}$ by its automorphism group, Invent. Math., 41(1977), 253-257

[40] J. Yu, Multitypes of convex domains, Indiana Univ. Math. J., 41(1992), 837-849

[41] J. Yu, Weighted boundary limits of the generalized Kobayashi-Royden metrics on weakly pseudoconvex domains, Trans. Amer. Math. Soc., 347(1995), 587-614

Department of Mathematics, Indian Institute of Science, Bangalore 560 012, India

E-mail address: mittal@math.iisc.ernet.in, kverma@math.iisc.ernet.in 